\title{
SEARCH FOR NEW LIGHT BOSONS IN HIGGS BOSON DECAYS
}

\author{
A Dissertation \\ by \\ AYSEN TATARINOV \\ Submitted to the Office of Graduate and Professional Studies of \\ Texas A\&M University \\ in partial fulfillment of the requirements for the degree of \\ DOCTOR OF PHILOSOPHY
}

\begin{abstract}
Chair of Committee, Alexei Safonov
Committee Members, Bhaskar Dutta

Teruki Kamon

Peter McIntyre

Sherry Yennello

Head of Department, Peter McIntyre
\end{abstract}

August 2016

Major Subject: Physics

Copyright 2016 Aysen Tatarinov 


\section{ABSTRACT}

The Standard Model (SM) of particle physics is a theory that summarizes our current knowledge about the most fundamental constituents of matter and interactions between them. The Higgs boson is a central part of the SM as it provides masses to all other particles. After many decades of searches for it, on the 4th of July 2012 CERN announced a discovery of a new particle by CMS and ATLAS collaborations at the Large Hadron Collider (LHC). The properties of the new particle and the properties of the Higgs boson predicted by the SM are consistent at the level of precision of current measurements. The extensive physics program of the LHC experiments includes searches for new physics beyond the SM which complement further precision measurements of the properties of the new particle. These searches may lead to earlier confirmation that the particle is not the SM Higgs boson in case the new physics is found. This dissertation is dedicated to a search for decays of the newly discovered particle to pairs of new light bosons, each of which decays into the $\mu^{+} \mu^{-}$final state. The data set corresponds to an integrated luminosity of 5.3 $\mathrm{fb}^{-1}$ of proton-proton collisions at $\sqrt{s}=7 \mathrm{TeV}$, recorded by the CMS experiment at the LHC in 2011. No excess of events above the SM predictions is observed. A model-independent upper limit of $0.86 \mathrm{fb}$ on the product of the cross section times branching fraction times acceptance is set. The obtained limit is applicable to models where the new light bosons have lifetimes corresponding to flight distances within a few $\mathrm{cm}$ from the proton beamline before their decay. The recipe for application of the results to an arbitrary model with the same final state is provided. Interpretation of the results in the context of two benchmark models significantly surpasses the sensitivity of previous similar searches. 


\section{ACKNOWLEDGMENTS}

I would like to thank everybody who has helped and supported me along this long and exciting journey. You have made this dissertation possible and I'm very happy to have you in my life.

I would like to express my deep gratitude to my advisor, Alexei Safonov, for his continuous guidance and mentorship through my doctorate program, for setting a high bar for me and exposing me to a wide variety of challenging and interesting projects. I would like to thank Yuriy Pakhotin for numerous discussions, conversations, and rigorous documentation of our analyses. I would like to thank Jason Gilmore for an introduction into a world of electronics and all the fun we had at CERN, especially underground in the CMS experimental cavern. I'm thankful to Jim Pivarski for helping me to ramp up during my first year at Texas A\&M and shaping my way of thinking about physics and approaching any problem in general. I would like to thank Teruki Kamon and Bhaskar Dutta for their support and conversations, especially during the work on this dissertation.

I would like to thank all of my friends in Bryan and College Station who were there for me during my time at Texas A\&M. I will miss hanging out with you, our movie nights, hikes, and trips.

I would like to thank my advisors at Joint Institute of Nuclear Research in Dubna, Dmitry Madigozhin and Yury Potrebenikov, for their guidance and attention when I just started my career in experimental particle physics.

I'm grateful to my school teacher in Physics, Viktor Potapov, for his tireless mentorship, advice, and everything he taught me. Thanks to him I decided to study at Moscow Institute of Physics and Technology and major in Particle Physics. 
There are not enough words to express how deeply grateful I am to my parents, Peter and Tatiana, who have continuously supported all of the decisions I have made in my life and have provided everything they could to make sure that I had a chance to pursue my dreams. Without their love and support I wouldn't be where I am today. 


\section{TABLE OF CONTENTS}

Page

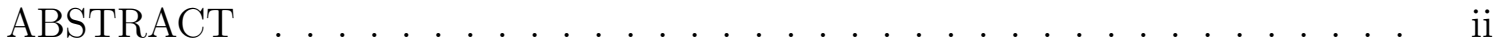

ACKNOWLEDGEMENTS ..................... . . iii

TABLE OF CONTENTS ..................... . . . . .

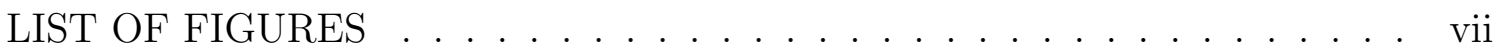

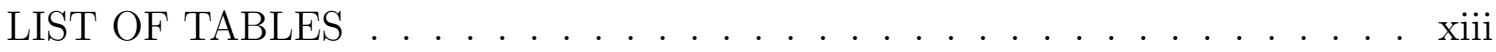

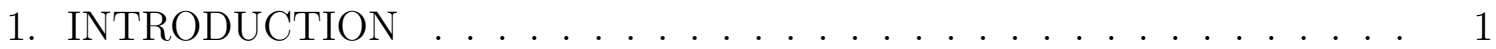

2. THEORETICAL FRAMEWORK .................. . . . 3

2.1 The Standard Model of Particle Physics . . . . . . . . . . . . . . . 3

2.1.1 Fundamental Forces and Elementary Particles . . . . . . . . . 3

2.1.2 Electroweak Theory and Quantum Chromodynamics . . . . . 5

2.1.3 Electroweak Symmetry Breaking and the Higgs Boson . . . . . 6

2.2 Beyond the Standard Model (BSM) . . . . . . . . . . . . . . . . 10

2.2.1 Extensions of the SM Predicting New Phenomena . . . . . . . 12

2.2.2 Models with Hidden Sectors . . . . . . . . . . . . . . . . . . . 14

3. EXPERIMENTAL APPARATUS . . . . . . . . . . . . . . . . 21

3.1 Large Hadron Collider . . . . . . . . . . . . . . . . . . . . . . . . . 21

3.2 The Compact Muon Solenoid (CMS) . . . . . . . . . . . . . . . 26

3.2.1 The CMS Coordinate System . . . . . . . . . . . . . . 28

3.2 .2 Tracking System . . . . . . . . . . . . . . . . . . . . 31

3.2.3 Electromagnetic Calorimeter . . . . . . . . . . . . . . . 34

3.2.4 Hadron Calorimeter . . . . . . . . . . . . . . . . . 36

3.2 .5 Muon System . . . . . . . . . . . . . . . . . 38

3.2.6 Trigger System . . . . . . . . . . . . . . . . . . 42

4. EVENT RECONSTRUCTION .................... . . . 44

4.1 Track Reconstruction . . . . . . . . . . . . . . . . . . 44 
4.2 Primary Vertex Reconstruction _ . . . . . . . . . . . . . . 46

4.3 Particle Identification . . . . . . . . . . . . . . . . . . . . . 47

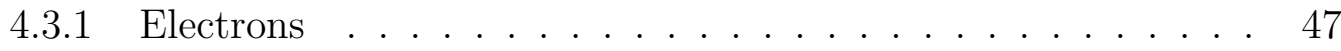

4.3 .2 Muons . . . . . . . . . . . . . . . . . . . . 49

4.3.3 Charged and Neutral Hadrons, Photons . . . . . . . . . . . 50

4.3 .4 Jets . . . . . . . . . . . . . . . . . . 51

4.3 .5 Tau Leptons . . . . . . . . . . . . . . . . . . . . 52

4.3.6 Missing Transverse Energy (MET) _ . . . . . . . . 53

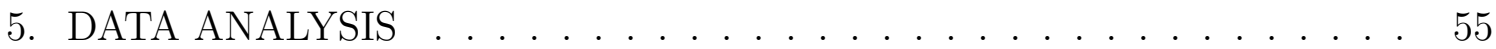

5.1 Analysis Strategy and Overview . . . . . . . . . . . . . 55

5.2 Analysis Selections . . . . . . . . . . . . . . . . . . . 59

5.2 .1 Offline Muon Candidate Selection . . . . . . . . . . . . . . 59

5.2 .2 Event Selection . . . . . . . . . . . . . . . . . . . 67

5.3 Selection Efficiency for Benchmark Scenarios . . . . . . . . . . . 81

5.4 Background Estimation . . . . . . . . . . . . . . . . . . . 85

$5.4 .1 \quad b \bar{b}$ Background Shape $\ldots \ldots \ldots \ldots \ldots \ldots . \ldots \ldots$

5.4 .2 Prompt Double $J / \psi$ Production . . . . . . . . . . . . . . . . 92

5.5 Systematic Uncertainties _. . . . . . . . . . . . . 96

5.5.1 Sources of Systematic Uncertainties . . . . . . . . . . . . . 97

5.5.2 Tag and Probe Studies . . . . . . . . . . . . . . . 102

5.6 Findings and Statistical Interpretation _ . . . . . . . . . . . 113

5.6.1 Model-Independent Limits . . . . . . . . . . . . . . . . . . . . 113

5.6.2 Limits in Benchmark Scenarios . . . . . . . . . . . . . 115

5.6 .3 Future Interpretations . . . . . . . . . . . . . 120

6. CONCLUSIONS . . . . . . . . . . . . . . . . . 123

REFERENCES . . . . . . . . . . . . . . . . . . . . . . . . 124 


\section{LIST OF FIGURES}

FIGURE

Page

2.1 Shape of the Higgs potential when $\mu^{2}<0$ and $\lambda>0 \ldots \ldots \ldots$

2.2 Main production modes of the Higgs boson using proton-proton collisions at LHC: gluon-gluon fusion (top left), vector boson fusion (top right), production with associated production of a vector boson (bottom left) or $t \bar{t}$ (bottom right). . . . . . . . . . . . . . . . .

2.3 The Standard Model Higgs boson production cross sections at the center-of-mass energy $\sqrt{s}=7 \mathrm{TeV}$ (top left) and $8 \mathrm{TeV}$ (top right), and branching ratios of various Higgs boson decay modes in the low mass (bottom left) and full (bottom right) search regions as a function of the Higgs boson mass. . . . . . . . . . . . . . . .

$2.490 \%$ confidence level limits in the parameter space of the mass of a new vector boson $m_{A^{\prime}}$ and its coupling $\epsilon$ to a photon from various experiments: beam dump experiments E137, E141, E774, the electron and muon anomalous magnetic moment $a_{\mu}$, KLOE, WASA-at-COSY, test run results from APEX and MAMI, estimates from BaBar results, supernova cooling. . . . . . . . . . . . . . . . 16

2.5 Branching ratios of decays into pairs of muons of new light vector (left, red dotted line) and scalar (right) bosons, $\gamma_{d}$ and $a$, respectively. . . .

2.6 Left: Feynman diagram of the NMSSM benchmark process $h_{1,2} \rightarrow$ $2 a_{1} \rightarrow 4 \mu \ldots \ldots \ldots \ldots \ldots \ldots \ldots \ldots \ldots$

3.1 The overview of the LHC circular underground tunnel with four LHC main experiments located at different collision points of the tunnel: CMS, ATLAS, LHCb, and ALICE. . . . . . . . . . . . . 22

3.2 Overview of CERN accelrator complex: protons used in LHC collisions originate from a single bottle of hydrogen and get accelerated when they pass through Linac2, the Proton Synchrotron Booster (PSB), the Proton Synchrotron (PS), and the Super Proton Synchrotron (SPS). 
3.3 Overview of LHC proton beams: beam 1 and beam 2 are directed clockwise and counterclockwise, respectively. . . . . . . . . . . . . 25

3.4 Total integrated luminosity of proton beam collisions delivered by the LHC in 2010 (green), 2011 (red), 2012 (blue). . . . . . . . . . . . 26

3.5 Total integrated luminosity of proton beam collisions delivered by the LHC (blue) and the integrated luminosity recorded by the CMS experiment (yellow) in 2011. . . . . . . . . . . . . . 27

3.6 Overview of the CMS detector and its subdetectors. . . . . . . . . 29

3.7 Transverse view of the CMS experiment illustrating the passage of different types of particles through the CMS detector and their interaction with its subdetectors. . . . . . . . . . . . . . . . . 30

3.8 The CMS coordinate system. . . . . . . . . . . . . . . 31

3.9 Layout of the CMS tracker: silicon pixel detector consisting of Tracker Pixel Barrel (TPB) and Tracker Pixel Endcap (TPE), silicon strip detector consisting of Tracker Inner Barrel (TIB), Tracker Inner Disks (TID), Tracker Outer Barrel (TOB), and Tracker EndCap (TEC).

3.10 The performance of the CMS tracking system for muons with transverse momentum of 1,10 , and $100 \mathrm{GeV}$ : track reconstruction efficiency (left) and track transverse momentum resolution (right) as a function of pseudorapidity. . . . . . . . . . . . . . . . . . . . .

3.11 Longitudinal view of a quarter of the CMS electromagnetic calorimeter (ECAL): the Barrel ECAL (EB), the Preshower (ES) in front of the Endcap ECAL $(\mathrm{EE}) . \ldots \ldots \ldots$

3.12 Longitudinal view of a quarter of the CMS hadronic calorimeter (HCAL): Barrel (HB), Endcap (HE), Outer (HO), and Forward (HF) hadronic calorimeters.

3.13 Longitudinal view of a quarter of the CMS detector illustrating the layout of the Muon System: Drift Tube chambers (DT), Cathode Strip Chambers (CSC), and Resistive Plate Chambers (RPC). . . . . . . 
3.14 Transverse view of one of the five wheels with Drift Tube chambers in the barrel region of the CMS. . . . . . . . . . . . . . . . . . . . 39

3.15 Muon transverse momentum resolution in pseudorapidity ranges of $|\eta|<0.8$ (left) and $1.2<|\eta|<2.4$ (right) using information from the tracker and the muon system separately or in combination. . . . . . . 40

3.16 Overview of the CMS Level 1 (L1) Trigger. . . . . . . . . . . . . . . . 42

4.1 Tracking efficiency measured with a tag-and-probe technique for muons from $\mathrm{Z}$ decays as a function of the muon $\eta$ (left) and the number of reconstructed primary vertices in the event (right) for data (black dots) and simulation (blue bands). . . . . . . . . . . . . . . . . . 46

4.2 Primary-vertex resolution in $\mathrm{x}$ (left) and in $\mathrm{z}$ (right) as a function of the number of tracks at the fitted vertex.

4.3 Primary-vertex reconstruction efficiency as a function of the number of tracks in a cluster, measured in minimum-bias data and in $\mathrm{MC}$

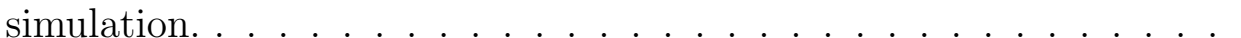

4.4 Distribution of PF MET for dijet events in data before (hollow markers) and after (solid markers) applying the cleaning algorithms compared to simulation (filled histograms). . . . . . . . . . . .

5.1 Left: sketch of a proton-proton collision with production of a pair of new light bosons that decay into two dimuons. . . . . . . . . . . 58

5.2 Comparison of the global muon (outside-in) (left) and the tracker muon (inside-out) (right) reconstruction efficiency as a function of the separation of the muon trajectories in the muon system (barrel). .

5.3 Comparison of the global muon (outside-in) (left) and the tracker muon (inside-out) (right) reconstruction efficiency as a function of the separation of the muon trajectories in the muon system (endcap).

5.4 Left: sketch of the two close muon tracks of opposite sign crossing pixel layer in adjacent elements forming single shared cluster. . . . . . 
5.5 Left: sketch introducing parameter $d$ - distance in $r$ - $\phi$ plane between the simulated hits of the muons in the pair on the first layer of the

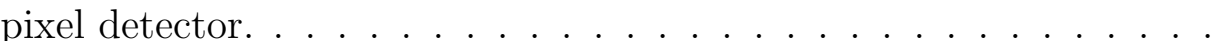

5.6 Distributions of the normalized $\chi^{2}$ for muon tracks that have only single shared reconstructed hit per dimuon or no assigned hit on the first layer of the pixel tracker. . . . . . . . . . . . . . . . . .

5.7 The HLT_Mu17_Mu8 per event trigger efficiencies measured using Monte Carlo simulation of NMSSM with $m_{h_{1}}=100 \mathrm{GeV}$ and $m_{a_{1}}$ $=2 \mathrm{GeV}$

5.8 Distribution of spacial separation $\Delta R$ between muons in a dimuon in NMSSM Monte Carlo samples with $m_{h_{1}}=100 \mathrm{GeV}$ and $m_{a_{1}}=0.25$, 0.5 , and $3 \mathrm{GeV}$

5.9 Left: sketch demonstrating reconstruction of the position of dimuon "production vertex" at the beamline, $z_{\mu \mu} \ldots \ldots$. . . . . . . . . 73

5.10 Four resonance mass peak fits in dimuon data: $\omega, \phi, J / \psi$, and $\psi^{\prime}$ in

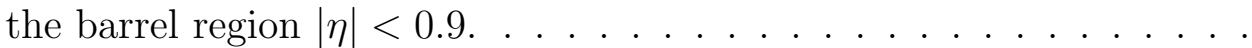

5.11 Four resonance mass peak fits in dimuon data: $\omega, \phi, J / \psi$, and $\psi^{\prime}$ in the endcap region $|\eta|>0.9 . \ldots \ldots$. . . . . . . . 76

5.12 Left: reconstructed invariant mass resolution as a function of the true mass for simulated dimuons (lines) and real resonances (points) in the barrel region $|\eta|<0.9$ as obtained using the fit to a Crystal Ball shape. 77

5.13 Left: sketch illustrating tracks used into the dimuon isolation. Right: dimuon isolation distribution for the reconstructed dimuons in NMSSM Monte Carlo sample with $m_{h_{1}}=100 \mathrm{GeV}$ and $m_{{\left(a_{1}\right.}_{1}}=2 \mathrm{GeV}$ and in events from background-enriched part of data.

5.14 Background templates of the mass distribution $S_{17}$ for "high- $p_{T}$ " type of dimuons (left) and the proper mix $S_{m i x}$ of the mass distributions $S_{17}$ and $S_{8}$ for "high- $p_{T}$ " and "low- $p_{T}$ " types of dimuons (right). . . 88

5.15 The 2D analytical template for distribution of the dimuon masses obtained using background-enriched data sample. . . . . . . . . . . 
5.16 Top, left: distribution of the mass of the "high- $p_{T}$ " type of dimuons from events in the control region (two dimuons per event; no isolation requirement; only the off-diagonal region) compared to the analytical template $S_{17} \ldots \ldots \ldots \ldots \ldots$

5.17 The distribution of the invariant mass $M$ of the two $J / \psi$ 's with $p_{T}^{J / \psi_{1,2}}>$ $3 \mathrm{GeV}$ in the "low- $p_{T}$ " sample. . . . . . . . . . . . . . . . . 94

5.18 The distribution of the invariant mass $M$ of the two $J / \psi$ 's with $p_{T}^{J / \psi_{1,2}}>$ $10 \mathrm{GeV}$ in the "low- $p_{T}$ " (left) and in the "high- $p_{T}$ " (right) samples.

5.19 Distributions of dimuon masses consistent with $J / \psi$ mass (from 2.8 to $3.4 \mathrm{GeV}$ ) containing passing, failing, and all probe muons with $p_{T}$ from 8 to $20 \mathrm{GeV}$ in $\mathrm{tag}$ and probe study of the analysis muon identification efficiency. . . . . . . . . . . . . . . . . . . 103

5.20 Distributions of dimuon masses consistent with $Z$ mass from 75 to $105 \mathrm{GeV}$ ) containing passing, failing, and all probe muons with $p_{T}$ from 20 to $40 \mathrm{GeV}$ in tag and probe study of the analysis muon identification efficiency. . . . . . . . . . . . . . . . . . . . . . 104

5.21 Distributions of dimuon masses consistent with $Z$ mass (from 75 to $105 \mathrm{GeV}$ ) containing passing, failing, and all probe muons with $p_{T}$ from 40 to $60 \mathrm{GeV}$ in tag and probe study of the analysis muon identification efficiency. . . . . . . . . . . . . . . . . 105

5.22 Distributions of dimuon masses consistent with $Z$ mass (from 60 to $120 \mathrm{GeV}$ ) containing passing, failing, and all probe muons in tag and probe study of HLT_Mu17_Mu8 trigger efficiency for high- $p_{T}$ probe

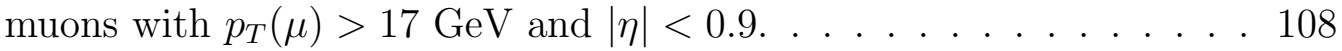

5.23 Distributions of dimuon masses consistent with $Z$ mass (from 60 to $120 \mathrm{GeV}$ ) containing passing, failing, and all probe muons in tag and probe study of HLT_Mu17_Mu8 trigger efficiency f or low- $p_{T}$ probe muons with $p_{T}(\mu)>8 \mathrm{GeV}$ and $|\eta|<2.4$. . . . . . . . . . . . 109

5.24 Distributions of dimuon masses consistent with $Z$ mass (from 75 to $105 \mathrm{GeV}$ ) containing passing, failing, and all probe muons in tag and probe study of the isolation requirement efficiency. . . . . . . . . . . . 112 
5.25 The distribution $m_{1}$ versus $m_{2}$ of masses of the dimuons for data events (shown as empty circles) surviving all selections except the requirement that these two masses fall into the diagonal signal region $m_{1} \approx m_{2}$ (outlined with dashed lines). . . . . . . . . . . . . 113

5.26 Left: The $95 \%$ CL upper limits as functions of $m_{h_{1}}$, for the NMSSM case, on $\sigma\left(p p \rightarrow h_{1,2} \rightarrow 2 a_{1}\right) \times \mathcal{B}^{2}\left(a_{1} \rightarrow 2 \mu\right)$ with $m_{a_{1}}=0.25 \mathrm{GeV}$ (dashed curve), $m_{a_{1}}=2 \mathrm{GeV}$ (dash-dotted curve) and $m_{a_{1}}=3.55$ $\mathrm{GeV}$ (dotted curve). . . . . . . . . . . . . 116

5.27 Left: The 95\% CL upper limits as functions of $m_{a_{1}}$, for the NMSSM case, on $\sigma\left(p p \rightarrow h_{1,2} \rightarrow 2 a_{1}\right) \times \mathcal{B}^{2}\left(a_{1} \rightarrow 2 \mu\right)$ with $m_{h_{1}}=90 \mathrm{GeV}$ (dashed curve), $m_{h_{1}}=125 \mathrm{GeV}$ (dash-dotted curve) and $m_{h_{1}}=150$ $\mathrm{GeV}$ (dotted curve). . . . . . . . . . . . . . 117

5.28 The $95 \%$ C.L. upper limit on the allowed NMSSM models consistent with LEP, WMAP, CLEO/BaBar measurements in the plane of $B\left(h_{1,2} \rightarrow a_{1} a_{1} \rightarrow 4 \mu\right)$ versus $\sigma\left(p p \rightarrow h_{1,2}\right)$ for $\sqrt{s}=7 \mathrm{TeV}$.

5.29 Left: distribution of $c \tau$, the real decay length of dark photons in signal sample with the nominal decay length $c \tau$ is set to $15 \mathrm{~cm}$. . . . . . . . 120 


\section{LIST OF TABLES}

TABLE

Page

2.1 Three generations of leptons and quarks. . . . . . . . . . .

5.1 Comparison of muon track related quality requirements in the analysis and the standard Soft Muon selection recommended by the CMS Muon POG shows full selection efficiency $\alpha_{R E C O}$ and efficiency of kinematic requirements on muon transverse momenta and pseudorapidities $\alpha_{G E N}$, as well as their ratio. . . . . . . . . . . . . .

5.2 Individual effects of changes (from analysis selection to the standard selection) in three specific muon track related quality requirements on full selection efficiency $\alpha_{R E C O}$ and efficiency of kinematic requirements on muon transverse momenta and pseudorapidities $\alpha_{G E N}$, as well as their ratio. . . . . . . . . . . . . . . . . . . .

5.3 List of the lowest threshold unprescaled inclusive non-isolated double muon triggers available during 2011 year. . . . . . . . . . . . . .

5.4 Acceptance efficiencies per event for several NMSSM Monte Carlo samples with $m_{h_{1}}=100 \mathrm{GeV}$ and $m_{a_{1}}$ varying from 0.25 to $3 \mathrm{GeV}$ as a function of the signal corridor width requirement. . . . . . . . . . 78

5.5 Efficiencies of the signal corridor requirement for the signal events $\left(\epsilon_{S}\right)$ in NMSSM sample with $m_{h_{1}}=100 \mathrm{GeV}$ and $m_{a_{1}}=3 \mathrm{GeV}$ and for the background events $\left(\epsilon_{B}\right),\left\langle R_{95 \% \text { C.L. }}\right\rangle$ is the estimate of the average expected $95 \%$ C.L. upper limit on the rate of signal events before applying the signal region requirement. . . . . . . . . . . . .

5.6 Efficiencies of the dimuons isolation requirement for dimuons in NMSSM Monte Carlo sample with $m_{h_{1}}=100 \mathrm{GeV}$ and $m_{\left(_{1}\right.}=2 \mathrm{GeV}$ and for background events $\epsilon_{B}^{d i-\mu}$, obtained using a background enriched sample with one dimuon and one orphan muon. . . . . . . . . . . . . . . . . 81

5.7 List of the acceptances and efficiencies (in \%) of the event selections for $m_{h_{1}}=100 \mathrm{GeV}$ and several representative benchmark values of $m_{a_{1}}$ (in $\mathrm{GeV}$ ) in Monte Carlo simulation of NMSSM signal. . . . . . . 
5.8 List of the acceptances and efficiencies (in \%) of the event selections for several representative benchmark sets of $m_{h_{1}}$ and $m_{a_{1}}$ (in $\mathrm{GeV}$ ) in Monte Carlo simulation of NMSSM signal. . . . . . . . . . . . . 83

5.9 List of the acceptances and efficiencies (in \%) of the event selections for several representative benchmark sets of $m_{h}, m_{n_{1}}, m_{n_{D}}, m_{\gamma_{D}}$ (in GeV) in Monte Carlo simulation of SUSY with hidden sector. . . . . . . . . 84

5.10 The ratio of the equivalent integrated luminosity $L_{M C}^{\text {low- }} p_{T}$ of the "low$p_{T}$ " sample of the simulated prompt $J / \psi J / \psi$ events and the correction factor $K_{\text {cor }}$ accounting for the apparent difference in the spectra of prompt double $J / \psi$ production in data and simulation. . . . . . . . .

5.11 The equivalent integrated luminosity of the "high- $p_{T}$ " sample calculated relative to the equivalent integrated luminosity of the "low- $p_{T}$ " sample in different bins of $M$ using the region with both $J / \psi$ 's having $p_{T}>10 \mathrm{GeV} \ldots \ldots \ldots \ldots \ldots \ldots$

5.12 The expected number of prompt double $J / \psi$ events passing selections of this analysis extrapolating the CMS measurements of the prompt double $J / \psi$ production in four bins of $M . \ldots$. . . . . . . . . . . . . . 96

5.13 Summary of the magnitude of systematic uncertainties. . . . . . . . 97

5.14 Summary of the correction (simulation to data) factors. . . . . . . . 102

5.15 Results of the T\&P study of the analysis muon identification algorithm with respect to muon tracking efficiency in the Silicon tracker in data and MC samples. . . . . . . . . . . . . . . 106

5.16 Illustration of the derivation of the scale factor for per-event efficiency using measured per leg efficiencies in data and simulation. . . . . . . 111

5.17 List of data events with two dimuons (and any number of orphan muons) surviving all analysis selections, but applying the requirement that the two masses fall into the off-diagonal region with the analysis

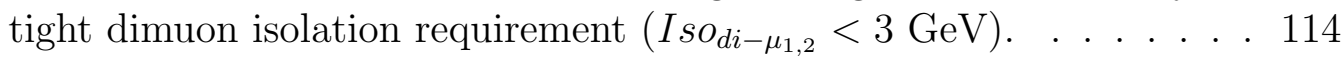


5.18 List of data events with two dimuons (and any number of orphan muons) surviving all analysis selections, but applying the requirement that the two masses fall into the off-diagonal region and the analysis loose dimuon isolation requirement for $\left(3<I s o_{d i-\mu_{1,2}}<8 \mathrm{GeV}\right)$. . . 115

5.19 Dependence of $\alpha_{\text {reco }} / \alpha_{\text {gen }}$ on $c \tau$, the nominal decay length of dark photons. . . . . . . . . . . . . . . . . . 121 


\section{INTRODUCTION}

For hundreds of years philosophers and scientists seek answers to the most fundamental questions in science: What are the smallest building blocks of the universe around us? How do they interact with each other? Elementary particle physics is a field of science that studies these questions. In the last century it has undergone a rapid development starting from the discovery of electron in 1897 [1] to discoveries

of quarks $[2,3,4,5,6,7]$, gluon $[8,9], \mathrm{W}$ and $\mathrm{Z}$ bosons $[10,11,12,13]$, and, finally, the Higgs boson in $2012[14,15]$.

The theoretical framework of particle physics, the Standard Model [16], has been largely established by 1970s. It has been a major success at explaining many physical phenomena observed at particle physics experiments and even predicting new particles before their discovery. Numerous precision measurements and experimental results confirm predictions of the Standard Model.

Despite all successes of the Standard Model, it has a number of limitations. For example, it doesn't include gravity and doesn't provide explanation for it, dark matter and dark energy, neutrino oscillations, and some of its parameters require severe fine tuning. The models beyond the Standard Model, either its extensions or completely novel models, aim at mitigating these and other limitations of the Standard Model. Searches for new physics complement precision measurements of phenomena predicted by the Standard Model, the latter are important as observed deviations from the Standard Model predictions would signify presence of new physics.

Higgs Boson plays the central role in the Standard Model as the Higgs mechanism $[17,18,19,20,21]$ provides masses of all known elementary particles via the electroweak symmetry breaking. After discovery of the top quark in 1995 [7], the 
Higgs boson has remained the last undiscovered particle of the Standard Model until after several decades of searches for it, on the 4th of July 2012 the discovery of a new particle consistent with the Standard Model Higgs boson has been jointly announced by the CMS [22] and ATLAS [23] experiments that study proton-proton collisions at the Large Hadron Collider (LHC) [24]. It is yet to be confirmed whether it is the Higgs boson of the Standard Model or a Higgs boson predicted in many models beyond the Standard Model.

This dissertation presents a search for evidence of new physics beyond the Standard Model by studying the decays of this newly discovered particle to pairs of new light bosons, where each of the new bosons decays into the $\mu^{+} \mu^{-}$final state. The search is motivated by a range of models beyond the Standard Model. The search has been performed using the data set of proton-proton collisions recorded by the CMS experiment at the Large Hadron Collider in 2011 with center-of-mass energy of $\sqrt{s}=7 \mathrm{TeV}$. The integrated luminosity of the data set corresponds to $5.3 \mathrm{fb}^{-1}$.

The structure of the dissertation is as follows. Chapter 2 provides a brief review of the Standard Model and models beyond it, and introduces the models used for benchmarking the performance of the search. Chapter 3 describes the Large Hadron Collider and the apparatus of the CMS experiment, including its sub-detectors, trigger and data acquisition systems. Chapter 4 briefly covers the reconstruction of physics objects at CMS with an emphasis on the muon reconstruction that this study relies on. Chapter 5 describes technical details of the data analysis and presents the results and their interpretations. 


\section{THEORETICAL FRAMEWORK}

\subsection{The Standard Model of Particle Physics}

The Standard Model of particle physics is a theory that summarizes our current knowledge about the most fundamental constituents of matter and fundamental interactions between them. Its current framework has been formed in 1960-1970s. Three main cornerstones that have contributed to the formulation of the Standard Model are:

- Unification of electromagnetic and weak interactions in a single framework by Glashow, Salam, Weinberg [25, 26, 27].

- Development of Quantum Chromodynamics (QCD) to describe strong interactions $[28,29,30,31,32]$.

- Introduction of the Higgs mechanism to explain masses of gauge bosons of weak interaction by Brout, Englert, Higgs, Guralnik, Hagen, and Kibble [17, 18, 19, $20,21]$.

In this section we briefly review the Standard Model.

\subsubsection{Fundamental Forces and Elementary Particles}

The Standard Model describes three out of four fundamental interactions: weak, strong, and electromagnetic (the gravitational interaction is not included in the Standard Model, however, it is not relevant at the scales of the particle physics). Each of these three interactions is mediated by corresponding spin-1 particles (bosons).

All known kinds of matter consist of spin- $1 / 2$ particles (fermions) grouped into three generations of quarks and leptons. Table 2.1 summarizes their properties. 
Quarks have always been observed in bound states of several quarks referred to as hadrons. Most common examples in nature are protons, neutrons, pions.

\begin{tabular}{|c|c|c|c|c|c|}
\hline & \multicolumn{3}{|c|}{$\begin{array}{c}\text { Fermions }(\text { Spin-1/2) } \\
\text { Generations }\end{array}$} & \multirow[t]{2}{*}{ Interactions } \\
\hline & & $\mathrm{I}$ & II & III & \\
\hline \multirow[t]{2}{*}{ Leptons } & $\begin{array}{l}\mathrm{Q}=0 \\
\text { Mass }\end{array}$ & $\begin{array}{c}\nu_{e} \\
<2 \mathrm{eV}\end{array}$ & $\begin{array}{c}\nu_{\mu} \\
<0.19 \mathrm{MeV}\end{array}$ & $\begin{array}{c}\nu_{\tau} \\
<18.2 \mathrm{MeV}\end{array}$ & Weak \\
\hline & $\begin{array}{l}\mathrm{Q}=-1 \\
\text { Mass }\end{array}$ & $\begin{array}{c}\mathrm{e} \\
511 \mathrm{keV}\end{array}$ & $\begin{array}{c}\mu \\
106 \mathrm{MeV}\end{array}$ & $\begin{array}{c}\tau \\
1.8 \mathrm{GeV}\end{array}$ & $\begin{array}{c}\text { Weak } \\
\text { Electromagnetic }\end{array}$ \\
\hline \multirow[t]{2}{*}{ Quarks } & $\begin{array}{c}\mathrm{Q}=+2 / 3 \\
\text { Mass }\end{array}$ & $\begin{array}{c}\mathrm{u} \\
2.3 \mathrm{MeV}\end{array}$ & $\begin{array}{c}\mathrm{c} \\
1.3 \mathrm{GeV}\end{array}$ & $\begin{array}{c}\mathrm{t} \\
173 \mathrm{GeV}\end{array}$ & \multirow{2}{*}{$\begin{array}{c}\text { Weak } \\
\text { Electromagnetic } \\
\text { Strong }\end{array}$} \\
\hline & $\begin{array}{c}\mathrm{Q}=-1 / 3 \\
\text { Mass }\end{array}$ & $\begin{array}{c}\mathrm{d} \\
4.8 \mathrm{MeV}\end{array}$ & $\begin{array}{c}\mathrm{s} \\
95 \mathrm{MeV}\end{array}$ & $\begin{array}{c}\mathrm{b} \\
4.2 \mathrm{GeV}\end{array}$ & \\
\hline
\end{tabular}

Table 2.1: Three generations of leptons and quarks.

Standard Model is a gauge theory invariant under local gauge transformations of $S U(2)_{L} \otimes U(1)_{Y} \otimes S U(3)_{C}$ symmetry group [33, 16]. Here, $S U(2)_{L} \otimes U(1)_{Y}$ is a symmetry group of the electroweak theory introduced by Glashow, Salam, and Weinberg $[25,26,27]$ to describe weak and electromagnetic interactions, $S U(3)_{C}$ is a symmetry group of the Quantum Chromodynamics (QCD) [28, 29, 30, 31, 32], which describes the strong interaction.

Invariance under local gauge transformations is achieved by introduction of one or more additional physical fields that compensate the change in the Lagrangian density. This can be illustrated in case of quantum electrodynamics (QED) that becomes invariant under local $U(1)_{Q}$ transformations after addition of a photon field. The Dirac Lagrangian density describing the constituents of matter by a fermionic field $\psi$ : 


$$
\mathcal{L}_{D}=\bar{\psi}\left(i \gamma^{\mu} \partial_{\mu}-m\right) \psi
$$

after the $\partial_{\mu}$ derivative is substituted by the covariant derivative $D_{\mu}$ :

$$
D_{\mu}=\partial_{\mu}+i e A_{\mu}
$$

becomes:

$$
\mathcal{L}_{D}=\bar{\psi}\left(i \gamma^{\mu} \partial_{\mu}-e \gamma^{\mu} A^{\mu}-m\right) \psi
$$

where $A_{\mu}$ corresponds to a photon, a gauge boson mediating electromagnetic interaction.

\subsubsection{Electroweak Theory and Quantum Chromodynamics}

Implication of gauge invariance under local $S U(2)_{L} \otimes U(1)_{Y} \otimes S U(3)_{C}$ transformations introduces field $B_{\mu}$, three fields $W_{\mu}^{a}$, and eight fields $G_{F}^{b}$. The covariant derivative $D_{\mu}$ becomes:

$$
D_{\mu}=\partial_{\mu}+i g_{1} Y B_{\mu}^{a}+i g_{2} \frac{\sigma^{a}}{2} W_{\mu}^{a}+i g_{3} \frac{\lambda^{b}}{2} G_{\mu}^{b},
$$

where $g_{1}, g_{2}, g_{3}$ - electromagnetic, weak, and strong interaction couplings, respectively, Y - weak hypercharge, $\sigma^{a}$ and $\lambda^{b}$ - three Pauli matrices and eight Gell-Mann matrices [16] (representations of $\mathrm{SU}(2)$ and $\mathrm{SU}(3)$ symmetry groups, respectively). Here, gauge fields $B_{\mu}$ and $W_{\mu}^{a}$ are related to experimentally observable massive $W^{ \pm}$and $Z^{0}$ bosons and massless photon:

$$
W_{\mu}^{ \pm}=\frac{1}{2}\left(W_{\mu}^{1} \mp i W_{\mu}^{2}\right),
$$




$$
\begin{gathered}
Z_{\mu}=-\sin \theta_{W} B_{\mu}+\cos \theta_{W} W_{\mu}^{3}, \\
A_{\mu}=\cos \theta_{W} B_{\mu}+\sin \theta_{W} W_{\mu}^{3} .
\end{gathered}
$$

where $\theta_{W}$ is the Weinberg angle [16] defined as $\sin \theta_{W}=g_{1} / \sqrt{g_{1}^{2}+g_{2}^{2}}$.

\subsubsection{Electroweak Symmetry Breaking and the Higgs Boson}

One should note, that the Lagrangian density invariant under local $S U(2)_{L} \otimes$ $U(1)_{Y} \otimes S U(3)_{C}$ transformations may not include mass terms for fermions and gauge bosons. One would have to generate mass terms dynamically for experimentally massive fermions and bosons while preserving the $S U(2)_{L} \otimes U(1)_{Y}$ gauge invariance. One of the possible solutions has been proposed by Brout, Englert, Higgs, Guralnik, Hagen, and Kibble in 1964 [17, 18, 19, 20, 21] via the so called Brout-Englert-Higgs $(\mathrm{BEH})$ mechanism. The proposed mechanism introduces a complex scalar $S U(2)_{L}$ doublet:

$$
\Phi=\left(\begin{array}{c}
\phi^{+} \\
\phi^{0}
\end{array}\right)=\frac{1}{\sqrt{2}}\left(\begin{array}{c}
\phi_{1}+i \phi_{2} \\
\phi_{3}+i \phi_{4}
\end{array}\right)
$$

and a new term in Lagrangian density:

$$
\mathcal{L}=\left(D^{\mu} \Phi\right)^{\dagger} D_{\mu} \Phi-V\left(\Phi, \Phi^{*}\right)
$$

where the potential is chosen to have the following form:

$$
\left.V\left(\Phi, \Phi^{*}\right)=\mu^{2} \Phi^{\dagger} \Phi+\lambda() \Phi^{\dagger} \Phi\right)^{2}
$$

Fig. 2.1 illustrates the case when $\mu^{2}<0$ and $\lambda>0$, where the ground (vacuum) state is located on a circle in a complex plane. An arbitrary choice of the ground state 


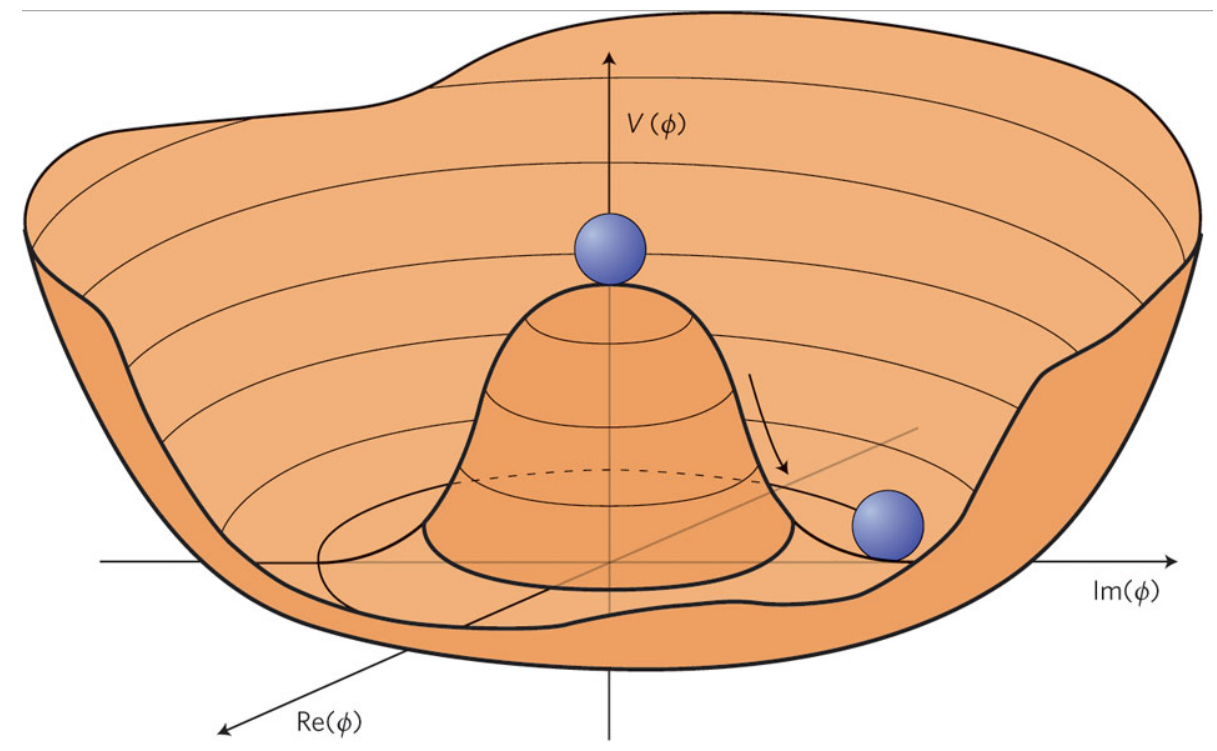

Figure 2.1: Shape of the Higgs potential when $\mu^{2}<0$ and $\lambda>0$ (from [34]).

breaks $S U(2)_{L} \otimes U(1)_{Y}$ symmetry (spontaneous electroweak symmetry breaking), but the Lagrangian density stays gauge invariant.

A random ground state that has been taken after the electroweak symmetry breaking can be expressed as:

$$
\Phi_{0}=\frac{1}{\sqrt{2}}\left(\begin{array}{c}
0 \\
v
\end{array}\right),
$$

where $v$ is the vacuum expectation value (v.e.v.) obtained by minimizing the potential:

$$
v=\sqrt{-\frac{\mu^{2}}{\lambda}} .
$$

Rewriting the scalar field as: 


$$
\Phi(x)^{\prime}=\frac{1}{\sqrt{2}}\left(\begin{array}{c}
0 \\
v+h(x)
\end{array}\right) .
$$

where $h(x)$ is associated with the scalar Higgs boson, and substituting it in the Lagrangian density leads to appearance of mass terms for massive gauge bosons. One should note that since the ground state $\Phi_{0}$ has no charged component, $U(1)_{Q}$ symmetry is preserved after the spontaneous breaking of $S U(2)_{L} \otimes U(1)_{Y}$ symmetry (as well as $S U(3)_{C}$ symmetry), leading to massless photons and gluons. Mass terms for fermions are introduced via Yukawa-type interactions of fermions with the Higgs field. Therefore, the Higgs coupling to fermions is proportional to fermion masses. This makes the Higgs boson preferentially couple to heavy leptons, which has a significant impact on the strategy of the search presented in this dissertation.

Fig. 2.2 illustrates the Higgs boson main production modes at the LHC as predicted by the Standard Model, and Fig. 2.3 shows their corresponding cross sections and branching ratios of the Higgs boson decay modes [36, 37]. The Higgs boson has remained a hypothetical particle for almost half a century until CERN has announced a discovery of a new boson with a mass near $125 \mathrm{GeV}$ in 2012 by two experiments, CMS and ATLAS, using proton-proton collisions at the LHC. The observed properties of the new boson are so far consistent with the Standard Model predictions within the uncertainties of the measurements. But the Higgs boson is also predicted by many extensions of the Standard Model, and it's yet to be established if the newly discovered boson is indeed the Higgs boson of the Standard Model. There are two complementary approaches to answering this question that are being pursued. The first one is to continue precision measurements of production and decay properties of the new boson. If a particular extension of the Standard Model predicts Higgs couplings similar to the Standard Model ones, this approach may require years of 


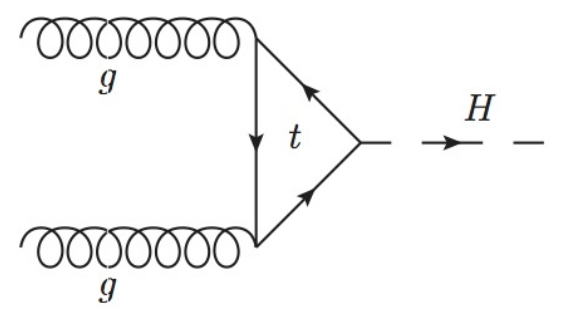

a)

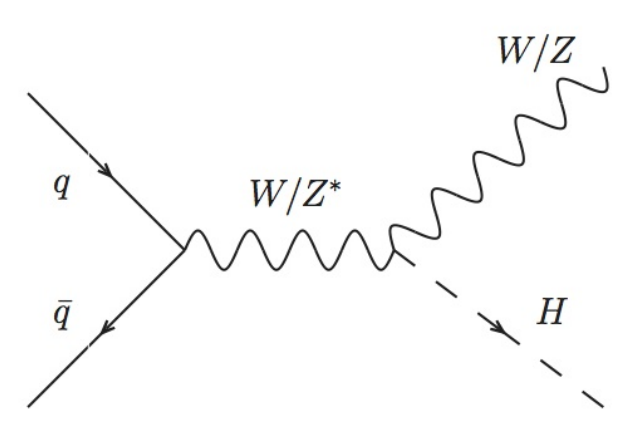

c)

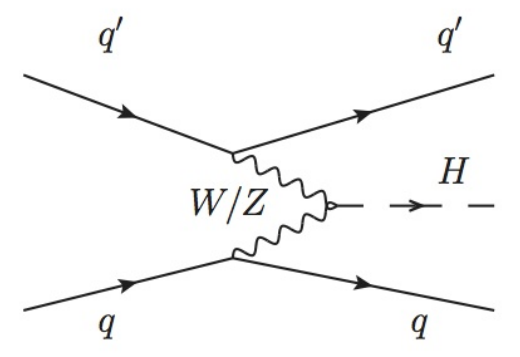

b)

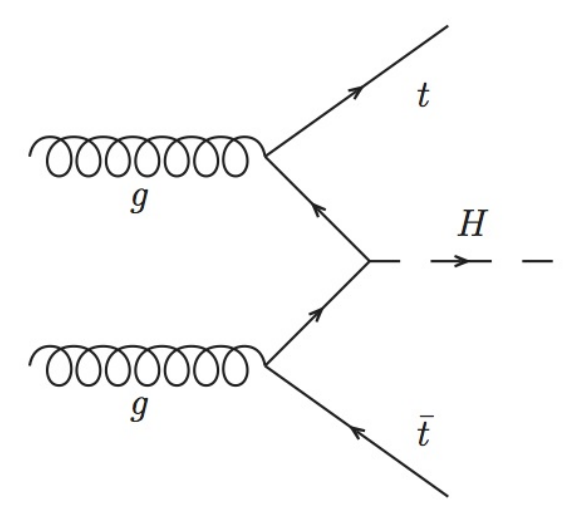

d)

Figure 2.2: Main production modes of the Higgs boson using proton-proton collisions at LHC: gluon-gluon fusion (top left), vector boson fusion (top right), production with associated production of a vector boson (bottom left) or $t \bar{t}$ (bottom right) (from $[35])$.

data collection. Another approach is to directly search for production and decay modes of non-Standard Model Higgs boson. If some search in the second approach has a striking experimental signature, it may lead to discovery or restriction of new physics scenarios with already existing data or with data to be collected in the near future. 

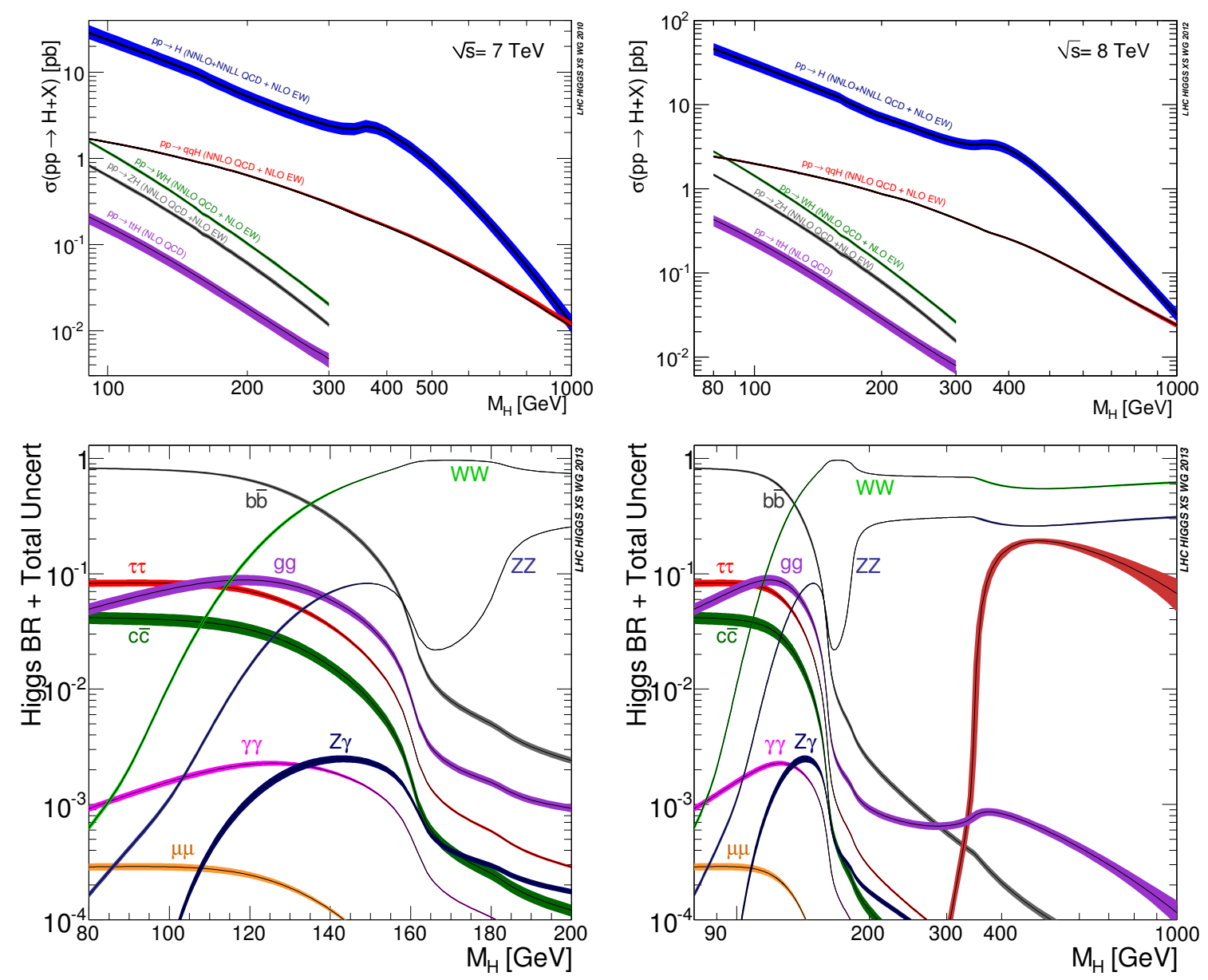

Figure 2.3: The Standard Model Higgs boson production cross sections at the centerof-mass energy $\sqrt{s}=7 \mathrm{TeV}$ (top left) and $8 \mathrm{TeV}$ (top right), and branching ratios of various Higgs boson decay modes in the low mass (bottom left) and full (bottom right) search regions as a function of the Higgs boson mass (from [36, 37]).

\subsection{Beyond the Standard Model (BSM)}

During last several decades the Standard Model has proven to be remarkably successful at describing and explaining many experimental results and observations at particle colliders and other experiments. Results of numerous precision measurements are consistent with the Standard Model predictions with an impressive 
accuracy. It has predicted existence of several particles before their discovery $\left(W^{ \pm}\right.$ and Z bosons, top quark, Higgs boson).

Despite all these successes, the Standard Model has a number of shortcomings both from experimental and theoretical points of view:

- It only describes three fundamental forces out of four as it makes no attempt to describe gravity.

- Observation of neutrino oscillations [38, 39] implies that neutrinos are not massless as predicted by the Standard Model.

- Astrophysical observations have first suggested existence of non-visible dark matter to account for discrepancies in motion of galaxies [40, 41]. Such explanation is also consistent with measurement of relic abundance in cosmological microwave background by satellite experiments $[42,43]$. The Standard Model doesn't provide any particle candidate for the dark matter.

- The radiative corrections to the Higgs boson mass are quadratically divergent at large energy scales. In order to suppress this divergence, Standard Model parameters require severe fine tuning (the so-called hierarchy problem [44]).

These and other issues are addressed by various extensions of the Standard Model and novel models of new physics. In this section we briefly review some of major classes of models beyond the Standard Model proposed to address these difficulties, we then switch our focus to a particular class of new physics models that contain hidden sectors: their motivation, current constraints, and typical experimental topologies at the LHC. 


\subsubsection{Extensions of the SM Predicting New Phenomena}

Below is a brief summary of some models beyond the Standard Model (this summary is not meant to be a comprehensive list):

- Supersymmetric (SUSY) models introduce a new symmetry connecting fermions and bosons: for each SM fermion there is a bosonic superpartner and for each SM boson there is a fermionic superpartner $[45,46]$. If masses of SM particles and their corresponding superpartners were the same, the radiative corrections to the Higgs boson mass exactly cancel each other (contributions from fermions and bosons have opposite sign) [47]. However, SM particles and their superpartners are not required to have equal masses. The SUSY models consistent with current experimental data still provide a viable solution to the hierarchy problem [44] if the mass splitting between SM particles and their superpartners is less than $O(1 \mathrm{TeV})$. Models with supersymmetry also provide a dark matter candidate as the lightest supersymmetric particle is stable if the R-parity is preserved [48]. The some of the most stringent constraints on existence of SUSY come from the LHC experiments. They have performed a wide range of searches for new particles predicted by SUSY models. Due to rich phenomenology of these models, the searches are designed to be inclusive and as generic as possible. The dominant production mechanisms are gluino-gluino, squarksquark, and squark-gluino production (squarks and gluinos are superpartners of quarks and gluinos) if at least some of the squarks or gluino are sufficiently light. Expected cross sections of these production mechanisms have had a large increase when the center-of-mass energy of proton-proton collisions at LHC experiments has increased from 8 to $13 \mathrm{TeV}$ in 2015. A typical SUSY search is for final states with energetic jets, which have been produced in decay chains 
of squarks and gluinos, and large missing transverse energy associated with the lightest supersymmetric particles (LSPs) produced in the end of decay chains [49].

- Models with large extra dimensions suggest existence of additional spatial dimensions beyond $3+1$ space-time dimensions. Addition of extra dimensions results in a reduced "effective" Planck energy scale, providing one solution to the hierarchy problem of the SM $[50,51]$. Most popular models are the Randall-Sundrum (RS) [52] and the Arkani-Hamed, Dimopulos, Dvali (ADD) [50] models that predict existence of gravitons and new heavy bosons. The most stringent limits on parameters of these models come from CMS and ATLAS experiments using searches for various final states including dileptons, dijets, and leptons or photons with large missing transverse energy [53].

- Compositeness models assume that leptons and quarks are composite and consist of new, yet unobserved, elementary particles [54]. These models may provide an explanation as to why there are three generations of leptons and quarks, and explain their charges and masses. At the scale of their constituent binding energies, new interactions are expected to appear, which are mainly described by the so-called Lagrangian contact terms [55]. The experiments at LHC, Tevatron, and SPS have searched for quark contact interactions using final states with jets, primarily studying their angular distributions [49].

- Some extensions of the SM suggest existence of leptoquarks, particles that carry both baryon and lepton numbers $[56,57]$. Collider experiments search for pair production of leptoquarks that arises from the leptoquark gauge interaction. The search signatures, defined by typical decays of leptoquarks, include leptons with high transverse momentum, jets with high transverse energy, and large 
missing transverse energy [49].

\subsubsection{Models with Hidden Sectors}

Models with hidden sectors hypothesize the existence of a hidden sector of new particles that do not interact with most SM particles or interact weakly, which so far allowed them to escape experimental detection. These models can be classified by the type of interaction between hidden and SM sectors: through vector, scalar, or neutrino portal [58]. Below we discuss more details and motivation for the first two types of portals.

One of the examples of the vector portal realization is kinetic mixing between the SM photon (hypercharge field $F_{Y}^{\mu \nu}$ ) and a new light vector boson (vector field $F_{\mu \nu}^{\prime}$ ) associated with new $\mathrm{U}(1)$ gauge group of the hidden sector [59]:

$$
\mathcal{L}_{\text {vector }}=\epsilon F_{\mu \nu}^{\prime} F_{Y}^{\mu \nu}
$$

Vector portals are well motivated from the perspective of string theory, particle phenomenology, cosmology, astrophysics:

- Hidden $\mathrm{U}(1)$ sectors arise in string theories via compactification of heterotic and type II strings [60, 61, 62].

- Existence of a new light vector particle can provide an attractive explanation $[63,64]$ of the observed discrepancy between measurement of muon anomalous magnetic moment in the E821 experiment at BNL [65] and the SM prediction.

- Cosmological theories with a hidden cosmic microwave background (hCMB) [66] predict a kinetic mixing between a new light boson and the SM photon. Current experimental constraints on the kinetic mixing favor the scenario that can be interpreted in the context of hCMB. 
- Several recent satellite experiments (PAMELA [67], Fermi LAT [68], AMS [69]) have observed a rising positron fraction as a function of the cosmis ray energy. An additional source of positrons can be provided by annihilation of dark matter into new light mediators [70] or decays of dark matter into the hidden sector [71] and then decays to visible sector.

- Dark matter self-interaction via light mediators $[72,73,74]$ can offer an explanation of the disagreement between the observation of flat density profiles of dwarf galaxies [75] and the prediction of steeper density profiles by collisionless DM-only simulations [76].

Hidden sectors naturally appear in well motivated models beyond the Standard Model. In the case of SUSY extended by a hidden sector, the lightest supersymmetric particle (LSP) can now decay into hidden sector particles, which will decay into the Standard Model particles through kinetic mixing with photon [77, 78]. If at the same time, the Higgs boson is at least two times heavier than the LSP, it can decay into LSPs: diverse physics motivation of models with hidden sectors strongly motivates this Higgs boson decay scenario.

The coupling constant between a photon and a new vector boson is constrained by previous experimental searches [79] summarized in Fig. 2.4.

A typical example of a scalar portal realization between the Standard Model and hidden sectors is the Next-to-Minimal Supersymmetric Standard Model (NMSSM) that extends the Minimal Supersymmetric Standard Model (MSSM) by an additional singlet superfield [95]. Among its attractive features are:

- Fine tuning of the NMSSM parameters and the little hierarchy problems are greatly diminished with respect to MSSM [96]. 


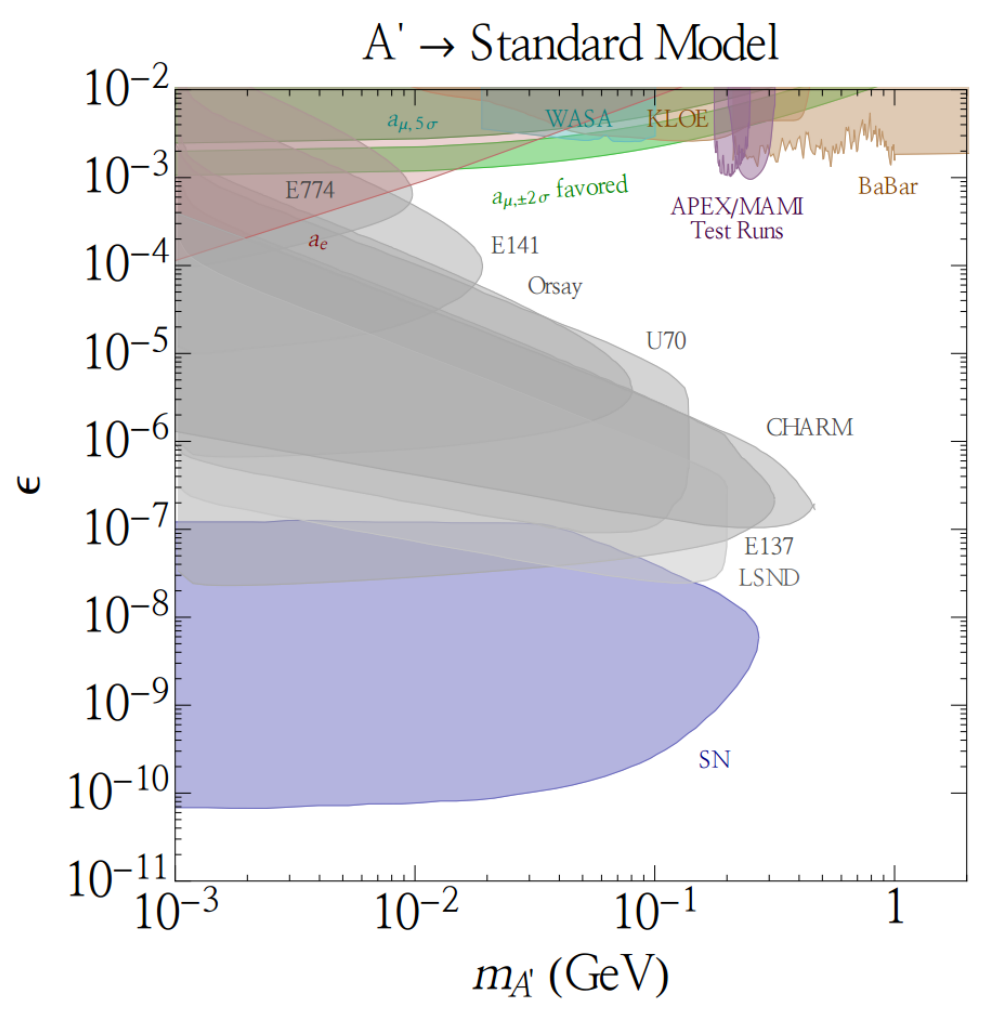

Figure 2.4: $90 \%$ confidence level limits in the parameter space of the mass of a new vector boson $m_{A^{\prime}}$ and its coupling $\epsilon$ to a photon from various experiments: beam dump experiments E137, E141, E774 [80, 81, 82, 83], the electron and muon anomalous magnetic moment $a_{\mu}[63,84,85]$, KLOE [86, 87], WASA-at-COSY [88], test run results from APEX [89] and MAMI [90], estimates from BaBar results [80, 91, 92], supernova cooling $[80,93,94] . A^{\prime}$ in the green band can explain observed discrepancy between the calculated and measured muon anomalous magnetic moment [64] (from [79]).

- NMSSM provides a solution to the so-called $\mu$-problem: the parameter $\mu$, which appears in the MSSM superpotential term $\mu H_{u} H_{d}$ and provides masses of higgsinos, fermionic superpartners of the Higgs bosons, has to be set manually in MSSM, but in NMSSM it dynamically acquires the EW scale to ensure $H_{u}$ and $H_{d}$ acquire a non-zero vacuum expectation value after EW symmetry breaking. [97]. 
The Higgs sector of NMSSM is more rich: three neutral CP-even Higgs bosons $h_{1,2,3}$, two neutral CP-odd Higgs bosons $a_{1,2}$, and two charged Higgs bosons $h^{ \pm}$. Higgs-to-Higgs decays within the sector, like $h_{1,2} \rightarrow a_{1} a_{1}$, can now have a substantial branching ratio, where either $h_{1}$ or $h_{2}$ can be the newly discovered boson with mass around $125 \mathrm{GeV}$ and $a_{1}$ boson can be light and have a coupling constant to the Standard Model fermions proportional to their mass [98, 99].

Previous searches motivated by NMSSM include:

- Searches for pair production of new light bosons decaying into dimuons at the Tevatron [100] and the LHC [101, 102] and other final states [103, 104].

- Searches for associated production of the light CP-odd scalar bosons at $e^{+} e^{-}$ colliders [105, 92] and the Tevatron [106].

- Direct $a_{1}$ production has been studied at the LHC [107], but this is heavily suppressed by the typically very weak couplings of the new bosons to SM particles.

Fig. 2.5 illustrates that the branching ratios of decays into pairs of muons of new light bosons (in both cases of scalar and vector portals between hidden and SM sectors) are enhanced when mass of the boson is between two masses of muons and two masses of $\tau$ leptons. This determines the search range of masses of the new light bosons, from 0.25 to $3.55 \mathrm{GeV}$, in the study presented in this dissertation.

\subsubsection{Experimental Challenges of Searching for Light Hidden Sectors}

Expected experimental signature of decays of new light bosons into muon pairs present a challenge from the experimental point of view. Decays of boosted light bosons into collimated muon pairs leave very close signals in the tracker and the muon 

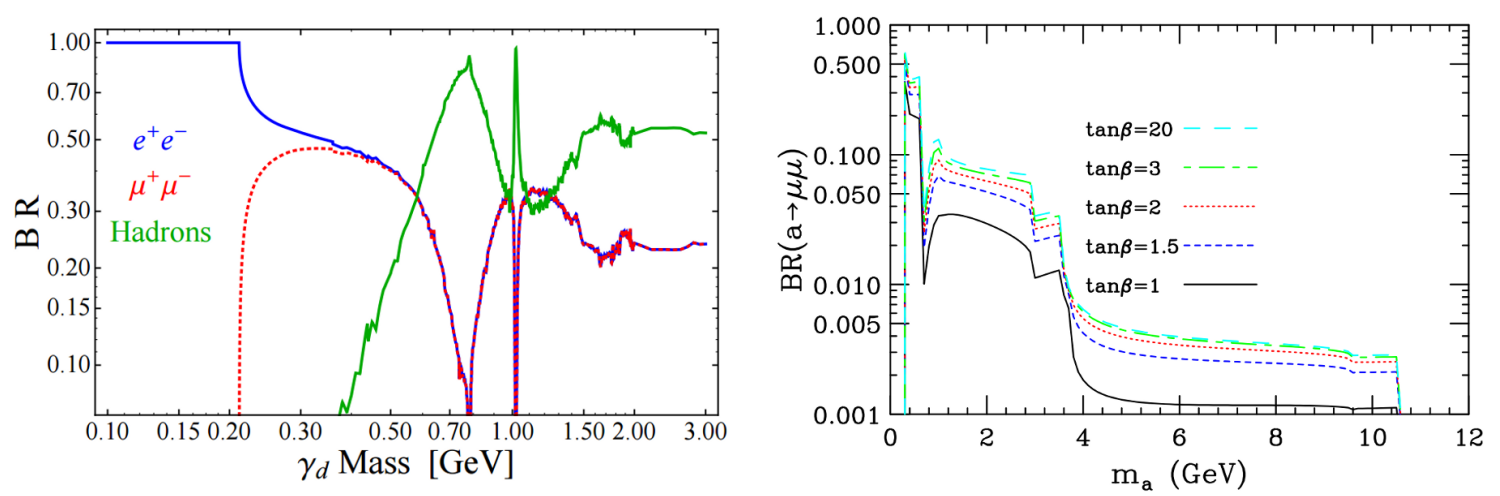

Figure 2.5: Branching ratios of decays into pairs of muons of new light vector (left, red dotted line) and scalar (right) bosons, $\gamma_{d}$ and $a$, respectively (from $[108,109]$ ).

system of the CMS experiment complicating the reconstruction of corresponding hits and tracks.

As it will be shown in Sec. 5.2, the standard selection criteria for muon candidates adopted at CMS are not suitable for our search. An important part of this analysis is dedicated to the optimization of these selection criteria aimed at efficient reconstruction of close-by muons both in the tracker and the muon system. To optimize the selections, we use simulation of two benchmark models based on two subclasses of models with hidden sectors (with vector and scalar portals between the hidden and Standard Model sectors). The following subsection provides more details of these benchmark models and their simulation.

\subsubsection{Benchmark Scenarios}

The benchmark scenario corresponding to a model with hidden sector connected to the SM sector via a vector is simulated with the MADGRAPH event generator [110] using SM Higgs boson production via gluon-gluon fusion gg $\rightarrow h_{S M}$, where the mass of $h_{S M}$ is set to the desired value for the h mass (within the range $90-150 \mathrm{GeV}$ ). The 

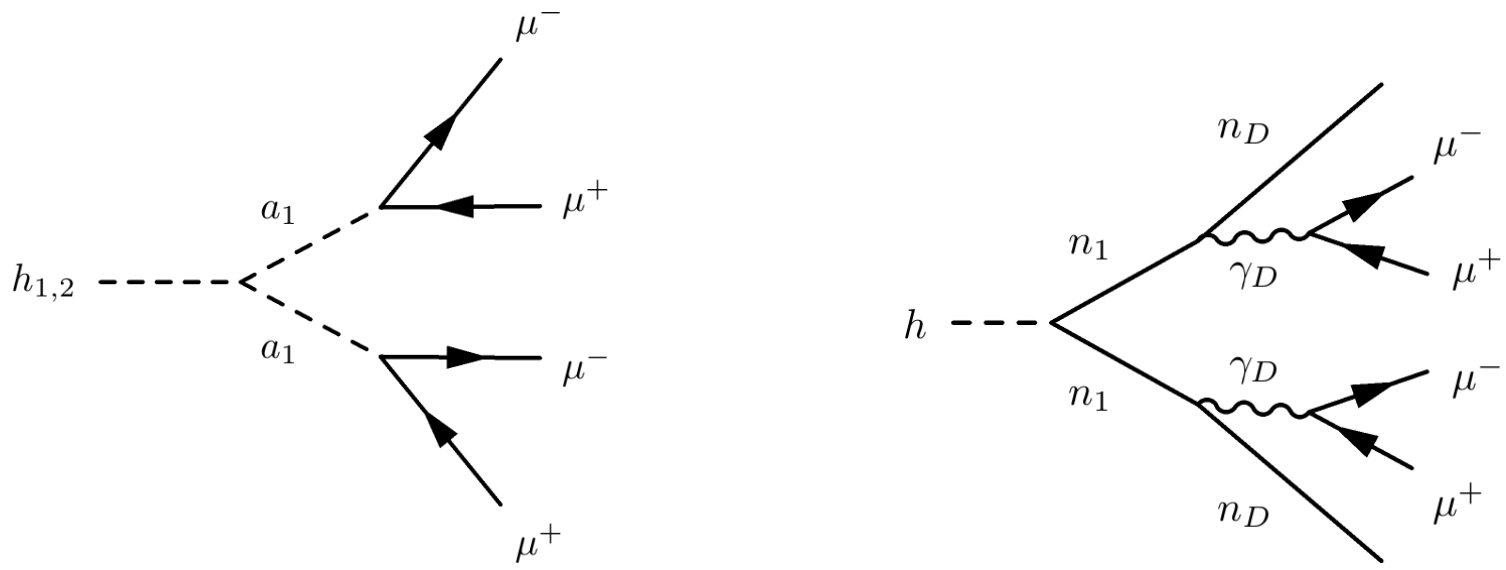

Figure 2.6: Left: Feynman diagram of the NMSSM benchmark process $h_{1,2} \rightarrow 2 a_{1} \rightarrow$ $4 \mu$. Right: Feynman diagram of the dark SUSY benchmark process $h \rightarrow 2 n_{1} \rightarrow$ $2 n_{D} 2 \gamma_{D} \rightarrow 2 n_{D} 4 \mu$.

BRIDGE software [111] is used to implement the new decay channel (Fig. 2.6(left)) that forces the Higgs bosons $h_{S M}$ to undergo a non-SM decay to a pair of neutralinos $\mathrm{n}_{1}$, each of which decays via $\mathrm{n}_{1} \rightarrow \mathrm{n}_{D}+\gamma_{D}$, where $m_{\mathrm{n}_{1}}=10 \mathrm{GeV}, m_{\mathrm{n}_{D}}=1 \mathrm{GeV}$ and $m_{\gamma_{D}}=0.4 \mathrm{GeV}$. Both dark photons $\gamma_{D}$ are forced to decay to two muons, while both dark neutralinos $\mathrm{n}_{D}$ escape detection.

The second benchmark scenario is based on NMSSM where the CP-even Higgs bosons $h_{1}$ (the SM-like Higgs) and $h_{2}$ can decay via $h_{1,2} \rightarrow a_{1} a_{1}$, where $a_{1}$ is a new light CP-odd Higgs. There are no strong indications as to what the mass of $a_{1}$ should be, so very low masses are allowed. If $2 m_{\mu}<m_{a_{1}}<2 m_{\tau}$ then it has a substantial branching fraction (about 17\% for $m\left(a_{1}\right) \sim 2 \mathrm{GeV}$, see Fig. 2.6(right)) for decays into muon pairs, $h_{1,2} \rightarrow a_{1} a_{1} \rightarrow 2 \mu 2 \mu$ making detection of $a_{1}$ in the muon channel viable at the LHC with already modest amounts of data.

The scenario is simulated with the PYTHIA 6 event generator [112] using MSSM Higgs boson production via gluon-gluon fusion $g g \rightarrow \mathrm{H}_{M S S M}^{0}$, where the Higgs bosons 
are forced to decay via $\mathrm{H}_{M S S M}^{0} \rightarrow 2 \mathrm{~A}_{M S S M}^{0}$. The masses of $\mathrm{H}_{M S S M}^{0}$ and $\mathrm{A}_{M S S M}^{0}$ are set to the desired values for the $h_{1}$ mass (within the range $86-150 \mathrm{GeV}$ ) and $\mathrm{a}_{1}$ mass (within the range $0.25-3.55 \mathrm{GeV}$ ), respectively. Both $\mathrm{A}_{M S S M}^{0}$ bosons are forced to decay to a pair of muons. The NMSSM Higgs production cross-sections are calculated using the SM Higgs production cross-sections $g g \rightarrow H_{S M}$ and $b \bar{b} \rightarrow H_{S M}$ with QCD-improved (running) Yukawa couplings [113] corrected for differences in coupling between NMSSM and SM using the NMSSMTools [114]:

$$
\begin{aligned}
\sigma\left(g g \rightarrow h_{1,2}\right) & =\sigma\left(g g \rightarrow H_{S M}\right) \frac{\operatorname{Br}\left(h_{1,2} \rightarrow g g\right) \Gamma^{t o t}\left(h_{1,2}\right)}{\operatorname{Br}\left(H_{S M} \rightarrow g g\right) \Gamma^{t o t}\left(H_{S M}\right)}, \\
\sigma\left(b \bar{b} \rightarrow h_{1,2}\right) & =\sigma\left(b \bar{b} \rightarrow H_{S M}\right)\left(\frac{Y_{b b h_{1,2}}}{Y_{b b H_{S M}}}\right)^{2} .
\end{aligned}
$$

where $\sigma\left(g g \rightarrow H_{S M}\right), \operatorname{Br}\left(H_{S M} \rightarrow g g\right)$ and $\Gamma^{t o t}\left(H_{S M}\right)$ are calculated using HIGLU [115], while $\operatorname{Br}\left(h_{1,2} \rightarrow g g\right), \Gamma^{t o t}\left(h_{1,2}\right)$, and the ratio of Yukawa couplings $Y_{b b h_{1}} / Y_{b b H_{S M}}$ are obtained using NMSSMTools.

All events of the benchmark signal samples are processed through a detailed simulation of the CMS detector [22] based on GEANT4 [116] and are reconstructed with the same algorithms as those used for data analysis. 


\section{EXPERIMENTAL APPARATUS}

\subsection{Large Hadron Collider}

The Large hadron Collider (LHC) [24] is the largest and most complex experimental facility for particle acceleration and collision ever built by mainkind. It has been built between 1998 and 2008 on the border of France and Switzerland at the European Organization for Nuclear Research (CERN). LHC is installed in a circular tunnel with a circumference of $27 \mathrm{~km}$ located between 50 and $170 \mathrm{~m}$ underground.

There are four main experiments located at different collision points of the tunnel (Fig. 3.1):

- CMS (Compact Muon Solenoid) [22] and ATLAS (A Toroidal LHC ApparatuS) [23] are two general purpose experiments with an extensive program of precision measurements testing the Standard Model and searches for new physics.

- LHCb (Large Hadron Collider beauty) [117] studies the physics of B-mesons and preforms precision measurements of CP-violation.

- ALICE (A Large Ion Collider Experiment) [118] is dedicated to physics of heavy ions and quark-gluon plasma.

The LHC is designed for collisions of protons and collisions of heavy ions, in this section we cover only the former case as this analysis is based on proton-proton collision data. All protons in LHC collisions originate from a single bottle of hydrogen (LHC proton beams require only $1 \mathrm{ng}$ of hydrogen per day). Hydrogen is fed into a cathode chamber of a duoplasmatron [119], where it dissociates and forms a plasma that is extracted through the anode to form a rough proton beam. Then it is sent 


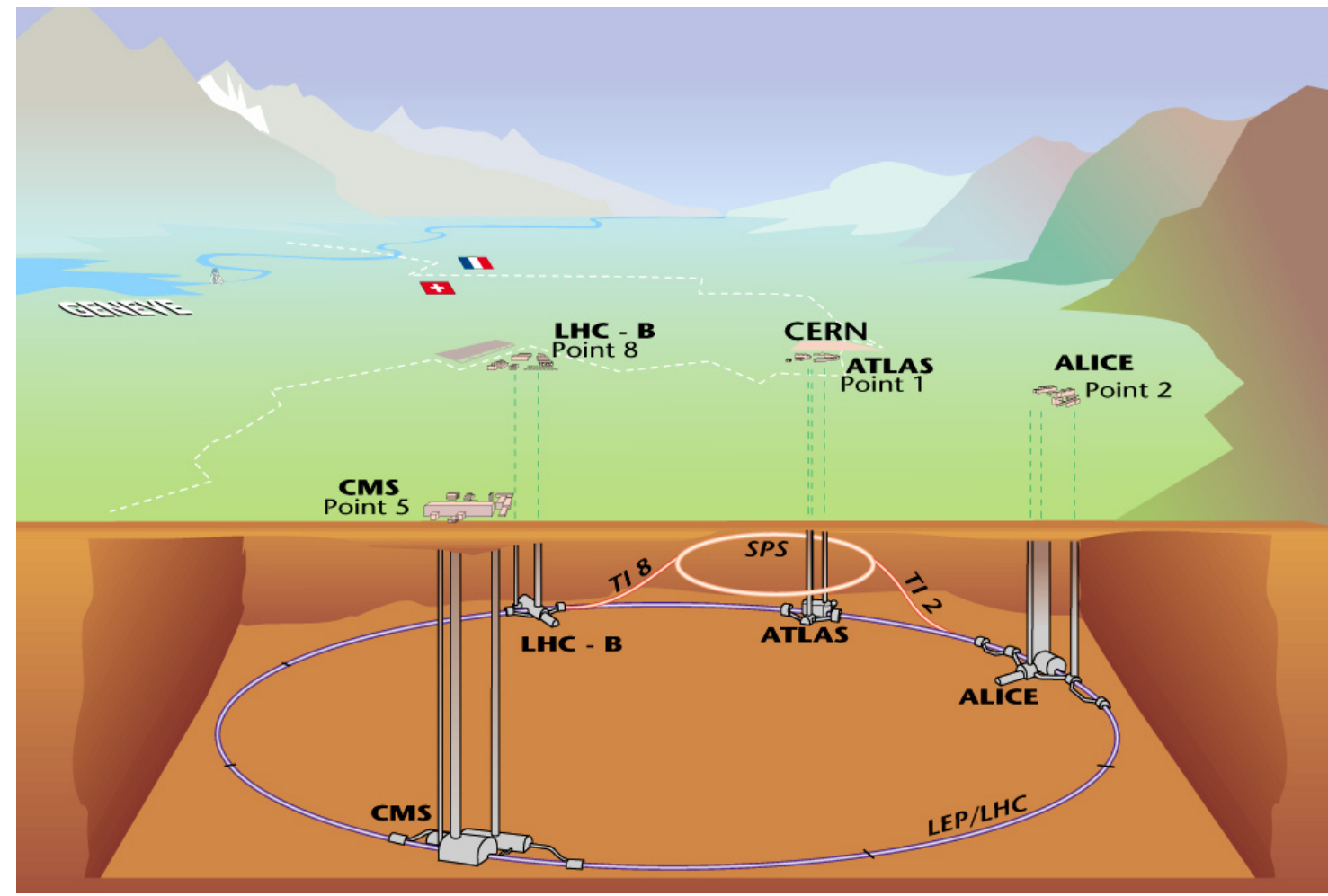

Figure 3.1: The overview of the LHC circular underground tunnel with four LHC main experiments located at different collision points of the tunnel: CMS, ATLAS, $\mathrm{LHCb}$, and ALICE.

through the the expansion cup and polarizer to shape the beam and stabilize its direction. The protons get clustered into bunches and accelerated in the following accelerator chain: to $50 \mathrm{MeV}$ in Linac2 linear accelerator, to $1.4 \mathrm{GeV}$ in the Proton Synchrotron Booster (PSB), to $26 \mathrm{GeV}$ in the Proton Synchrotron (PS), to $450 \mathrm{GeV}$ in the Super Proton Synchrotron (SPS) (Fig. 3.2).

After the SPS, proton bunches are injected into the LHC ring, where they travel inside two separate beam pipes (Fig. 3.3). Protons are accelerated within 16 radio frequency cavities by electromagnetic field oscillating at a frequency of $400 \mathrm{MHz}$. Proton beams are bent along the beam pipes by the field of 1232 superconducting 

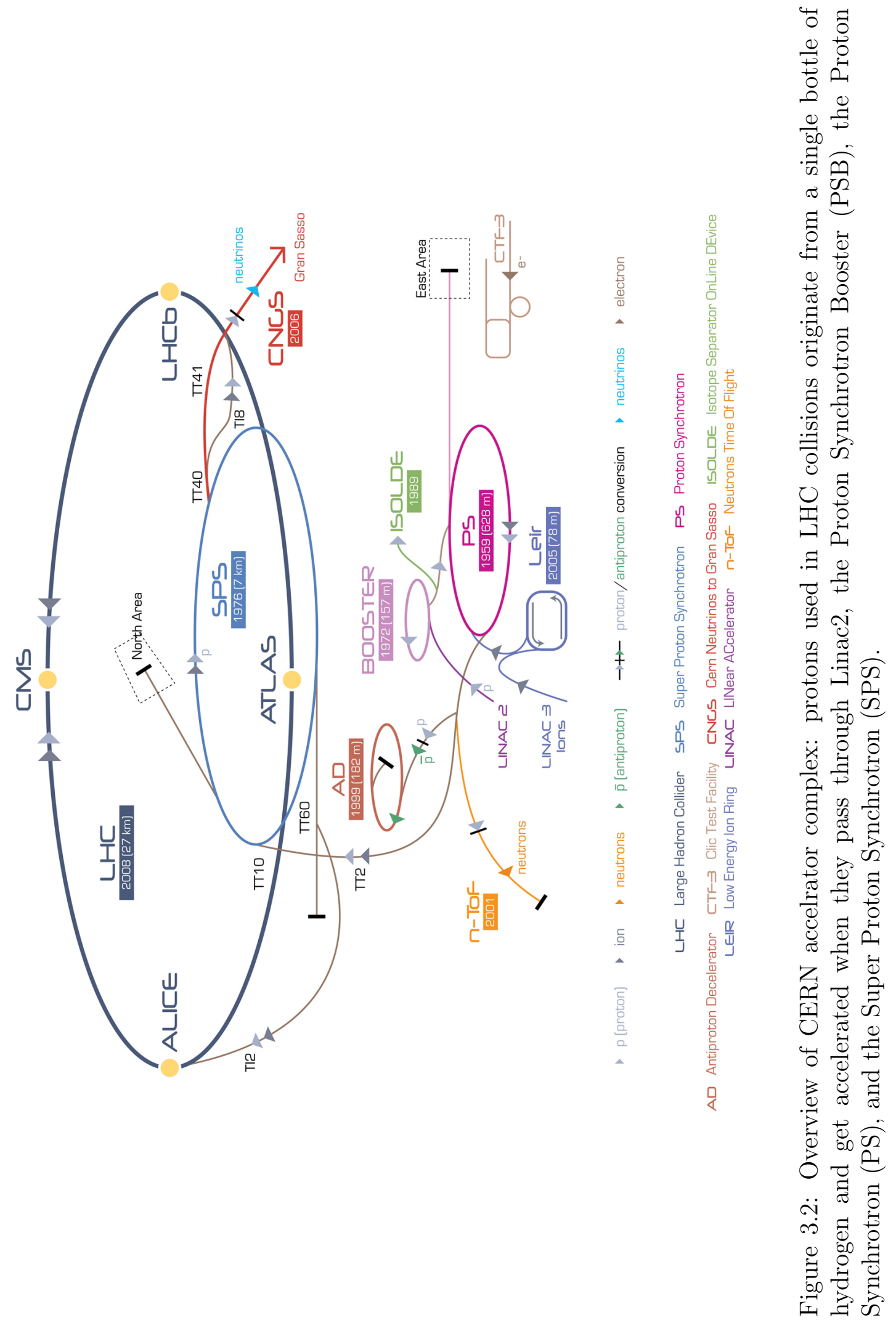
dipole magnets that are kept at the temperature of $1.9 \mathrm{~K}$ using $120 \mathrm{t}$ of superfluid helium. In addition to bending, beams are focused by 392 quadrupole magnets. After reaching the target energy, protons beams are adjusted to produce proton collisions in several experimental points along the LHC ring.

The start of the LHC was scheduled for September 2008, but due to an accident with some of the magnets it has been delayed until November 23, 2009, when the LHC started colliding proton beams with center-of-mass energy of $900 \mathrm{GeV}$. On March 30, 2010 the machine energy has been increased to $7 \mathrm{TeV}$ and has been kept constant during LHC operations in 2010 and 2011. In 2012 the center-of-mass energy has been increased to $8 \mathrm{TeV}$. From 2013 to early 2015 the LHC has been stopped to undergo technical upgrades of the machine and experiments, and in 2015 the center-of-mass energy of proton beam collisions has reached $13 \mathrm{TeV}$.

In addition to the energy of collisions, another important parameter of a particle collider is the collision luminosity. The rate of events $d N / d t$ of a given physical process is the product of collision luminosity $\mathcal{L}$ and the cross section $\sigma$ of that process:

$$
d N / d t=\mathcal{L} \cdot \sigma
$$

where the luminosity $\mathcal{L}$ depends on parameters of LHC and the optical properties of the focusing system at the interaction point [120]:

$$
\mathcal{L}=\frac{N^{2} n_{b} f \gamma}{4 \pi \epsilon_{n} \beta^{*}} F,
$$

where:

- $N$ : number of protons per bunch.

- $n_{b}$ : number of bunches in the LHC ring. 


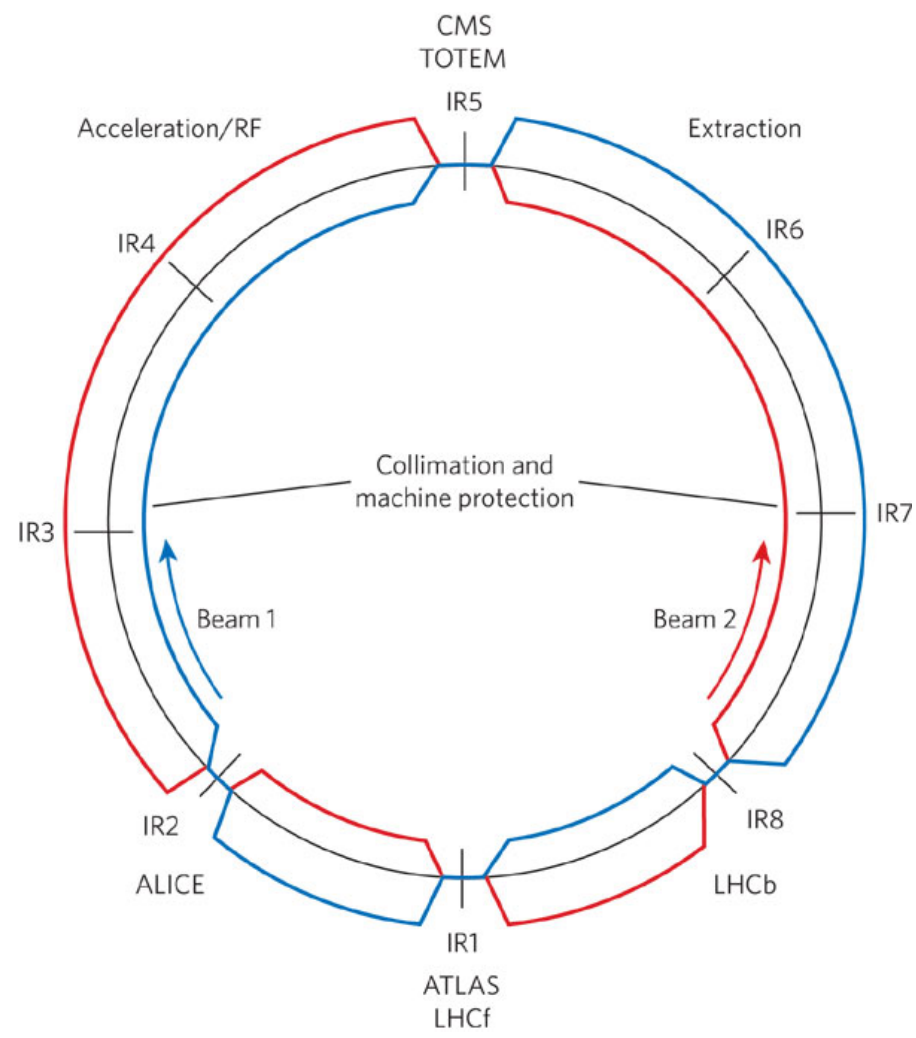

Figure 3.3: Overview of LHC proton beams: beam 1 and beam 2 are directed clockwise and counterclockwise, respectively. After reaching the target energy, protons are collided at several experimental points along the LHC ring.

- $f$ : the frequency of bunch revolution around the LHC ring.

- $\gamma$ : relativistic factor of protons.

- $\epsilon_{n}$ : normalized emittance of the proton beam.

- $\beta^{*}$ : beta function at the collision point.

- $F$ : the geometrical reduction factor due to the crossing angle.

During the $7 \mathrm{TeV}$ collisions run from 2010 to 2011 the luminosity has increased from $10^{32} \mathrm{~cm}^{-2} \mathrm{~s}^{-1}$ to $5 \cdot 10^{33} \mathrm{~cm}^{-2} \mathrm{~s}^{-1}$, and during 2012 (the LHC operating at the 
center-of-mass energy of $8 \mathrm{TeV}$ ) it has been further increased to $8 \cdot 10^{33} \mathrm{~cm}^{-2} \mathrm{~s}^{-1}$.

Fig. 3.4 and 3.5 summarize the total integrated luminosity delivered by the LHC and recorded by the CMS experiment during its running period from 2010 to 2012 . The analysis presented in this thesis uses data collected by the experiment in 2011 and certified for analysis using muons (the total integrated luminosity of $5.3 \mathrm{fb}^{-1}$ ).

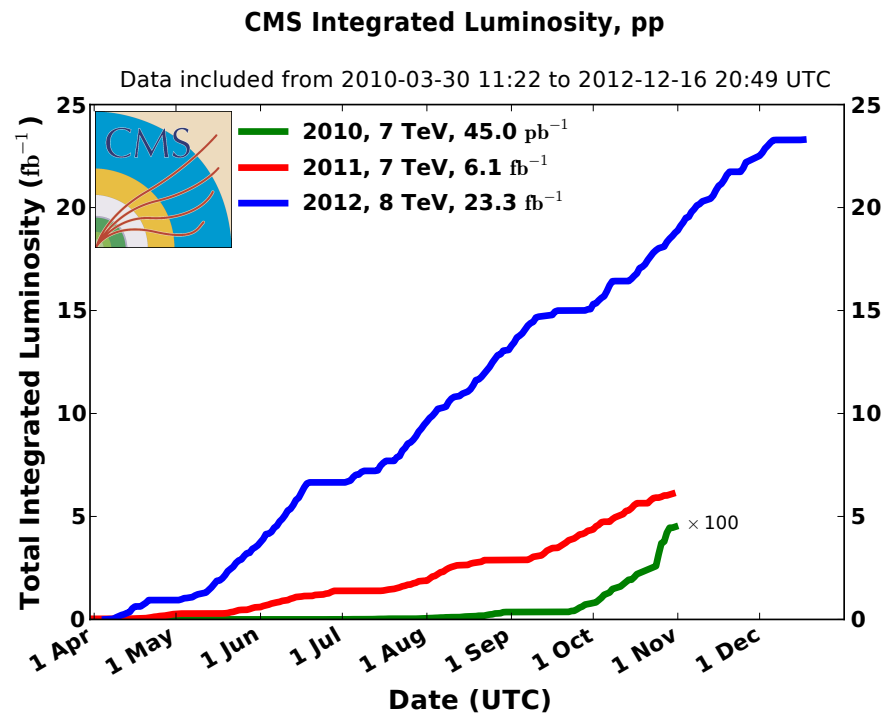

Figure 3.4: Total integrated luminosity of proton beam collisions delivered by the LHC in 2010 (green), 2011 (red), 2012 (blue).

\subsection{The Compact Muon Solenoid (CMS)}

The CMS experiment is one of two general purpose experiments at the LHC. The physics program of the experiment covers a wide range of goals: precision study of Standard Model processes, study of properties of the recently discovered Higgs boson, searches for physics beyond the Standard Model, and study of quark-gluon plasma. 


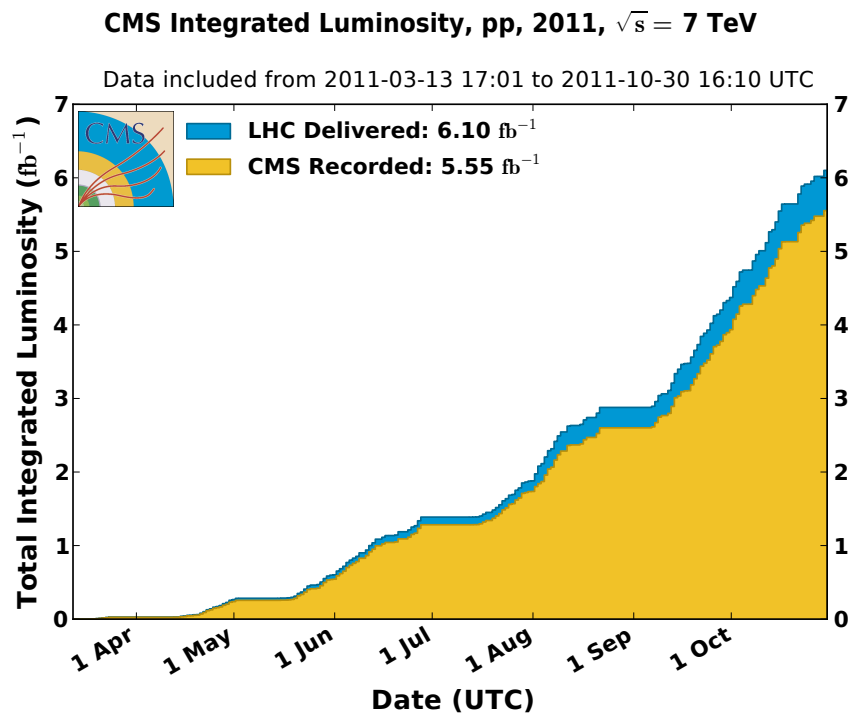

Figure 3.5: Total integrated luminosity of proton beam collisions delivered by the LHC (blue) and the integrated luminosity recorded by the CMS experiment (yellow) in 2011.

The CMS detector is located in the underground cavern near Cessy, France. It is the only one among other detectors at the LHC that has been assembled on the surface, then disassembled into 15 separate pieces, lowered down into the cavern, and reassembled again.

The CMS detector has a diameter of $15 \mathrm{~m}$ and length of $21.5 \mathrm{~m}$ and surrounds the proton beamline and the interaction point providing nearly full $4 \pi$ coverage of proton collisions. Among its subdetectors are (Fig. 3.6):

- The inner tracking system with highly efficient reconstruction of trajectories (tracks) and good momentum resolution of charged particles, and efficient reconstruction of primary and secondary vertices of proton-proton collisions.

- The electromagnetic calorimeter (ECAL) providing an accurate measurement of energy of photons and electrons. 
- The hadronic calorimeter (HCAL) measures the energy of neutral and charged hadrons and the missing transverse energy (MET).

- The muon system with high efficiency of muon identification and good resolution of muon momentum together with the tracker.

The central feature of the CMS detector is a high field superconducting solenoid with a nominal field of $3.8 \mathrm{~T}$, inner diameter of $6 \mathrm{~m}$ and length of $13 \mathrm{~m}$. Its weight including the iron return yoke confining its magnetic field $(10,000 \mathrm{t})$ is a large fraction of the total weight of the CMS detector $(12,500 \mathrm{t})$. The solenoid surrounds the inner tracker and both electromagnetic and hadronic calorimeters, while the muon system is outside of the solenoid. The iron return yoke with return field of the solenoid around $2 \mathrm{~T}$ is located within the muon system. High magnetic field passing through all subdetectors provides bending of high energy charged particles allowing a good resolution of their momentum measurement in the tracking subdetectors (inner tracker and muon system).

Fig. 3.7 illustrates typical interaction patterns of different kinds of particles within the CMS subdetectors. The following subsections will briefly review each subdetector and its properties.

\subsubsection{The CMS Coordinate System}

The CMS uses a right handed coordinate system with the origin at the nominal interaction point in the center of the CMS detector (Fig. 3.8). The x-axis points towards the center of the LHC ring, the y-axis points vertically upward, and the z-axis points along the beamline in the counterclockwise direction (looking from the top). The azimuthal angle $\phi$ is measured from the $\mathrm{x}$-axis in the $\mathrm{x}-\mathrm{y}$ plane, the polar angle $\theta$ is measured from the z-axis. However, at hadron colliders, it's more

convenient to replace $\theta$ by the rapidity $y=\frac{1}{2} \ln \left(\frac{E+p_{z}}{E-p_{z}}\right)$, which in relativistic limit 


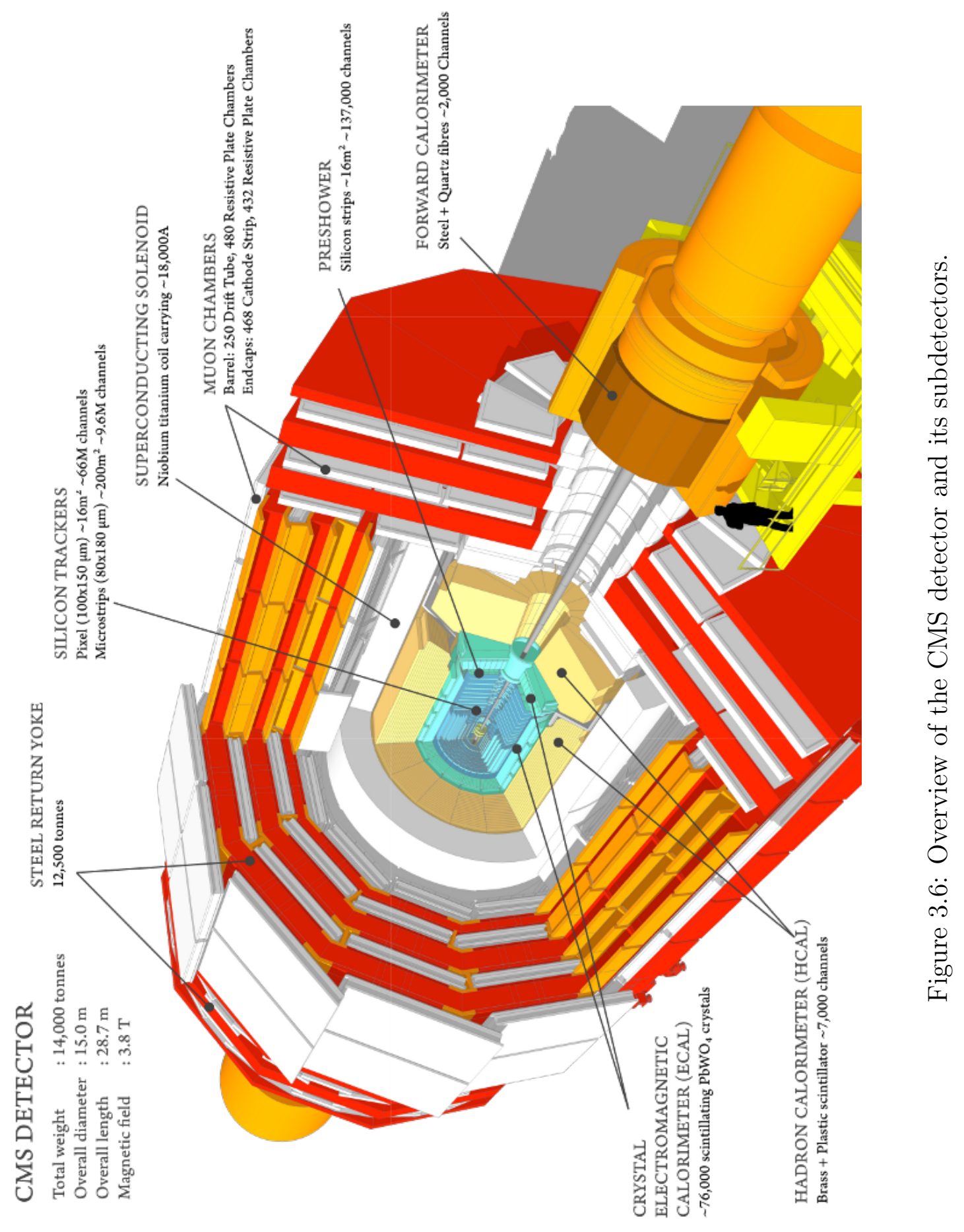




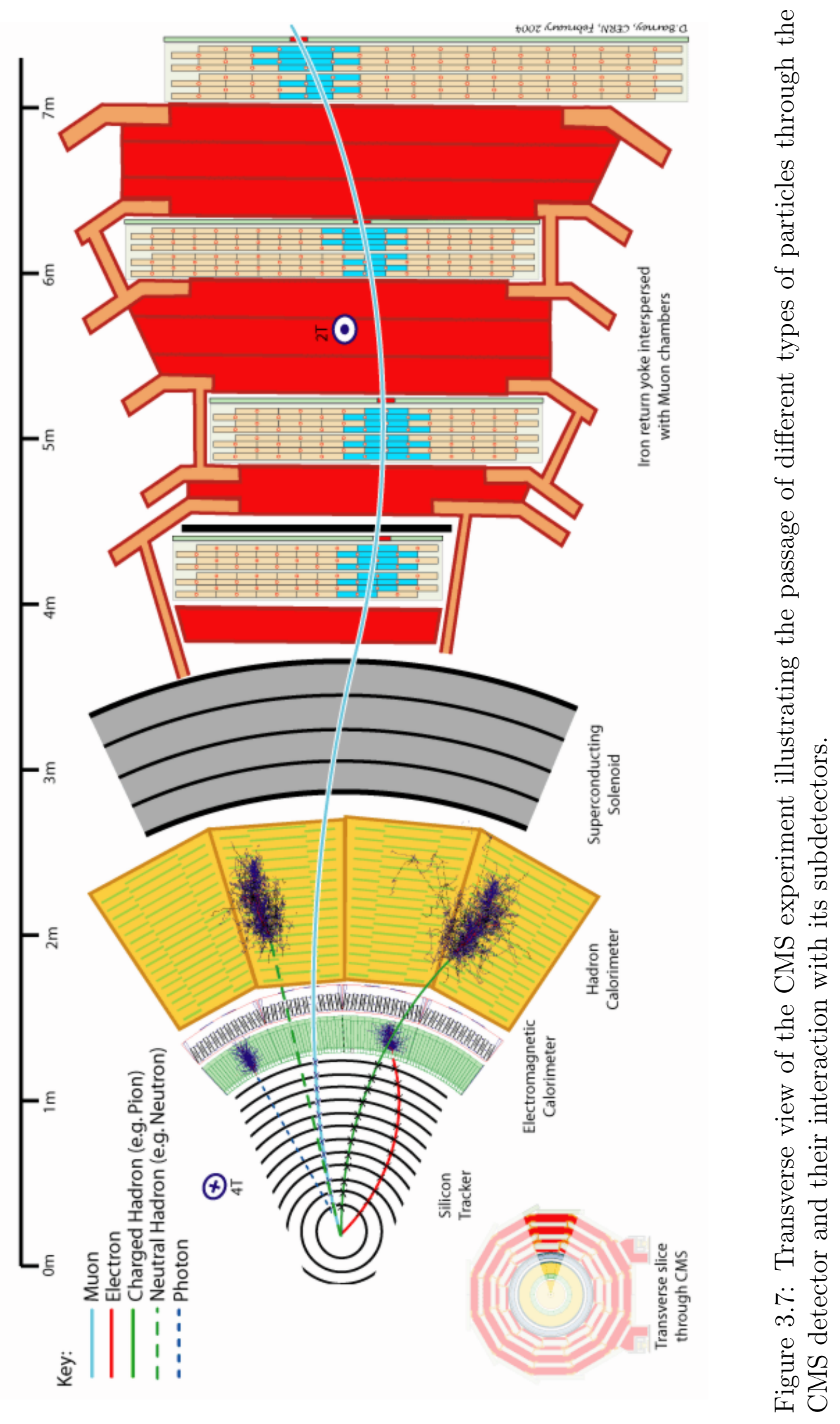




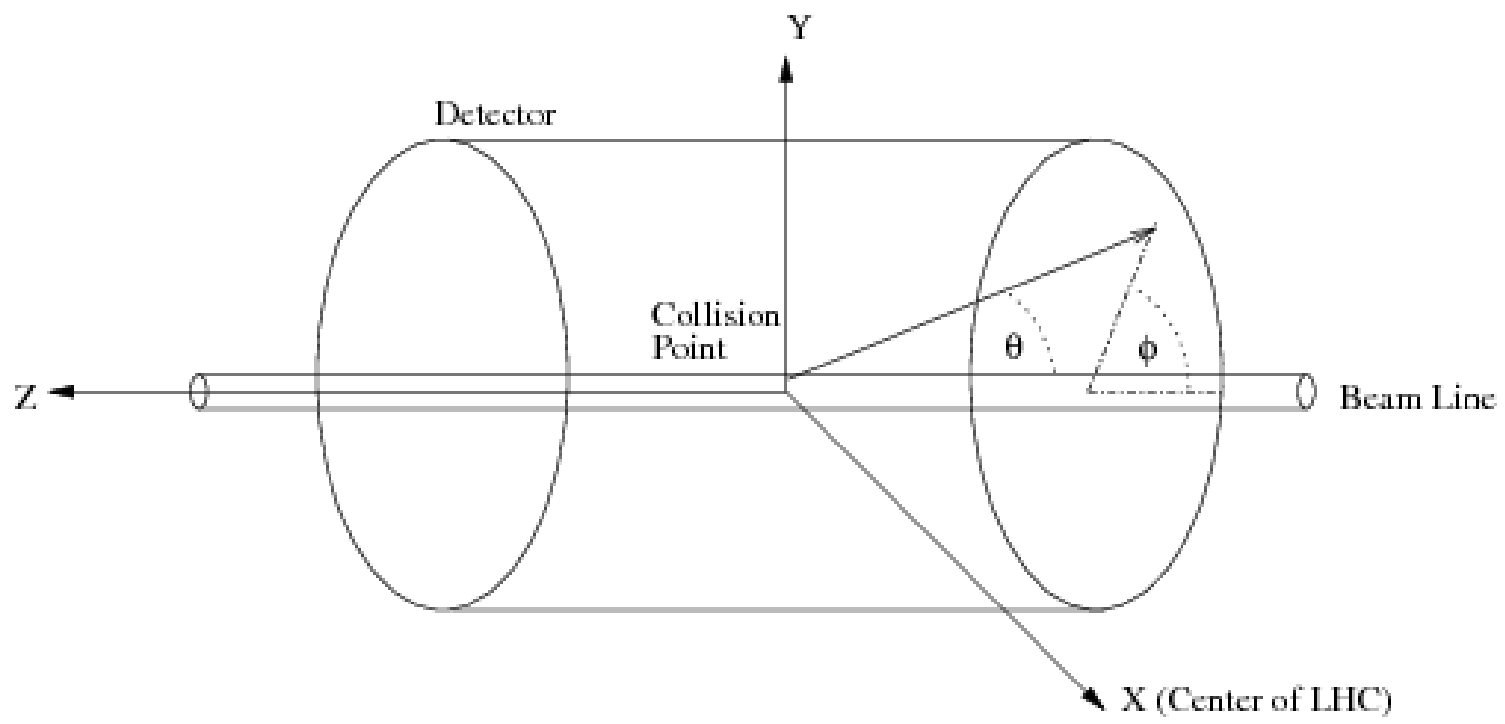

Figure 3.8: The CMS coordinate system.

$(E>>m)$ converges to pseudorapidity $\eta=-\ln \left(\tan \frac{\theta}{2}\right)$. The transverse momentum $p_{T}$ is defined as a projection of $\mathrm{p}$ to the $\mathrm{x}-\mathrm{y}$ plane: $p_{T}=\sqrt{p_{x}^{2}+p_{y}^{2}}$. The transverse energy is defined as $E_{T}=E \sin \theta$.

\subsubsection{Tracking System}

The data from the CMS tracking system (tracker) [121] is used to reconstruct trajectories of charged particles and measure their momenta, as well as reconstruct primary and secondary vertices in hadron collisions. Closeness of the tracker to the beamline exposes it to a large flux of particles with close-by trajectories given the high rate of collisions. This poses stringent requirements on the tracker parameters: it is required to have a high granularity, fast response, and be able to withstand severe radiation. The choice of silicon detector technology used in the tracker has been driven by these requirements. The tracker has a length of $5.8 \mathrm{~m}$, diameter of $1.1 \mathrm{~m}$, and consists of two parts: silicon pixel and silicon strip detectors. 


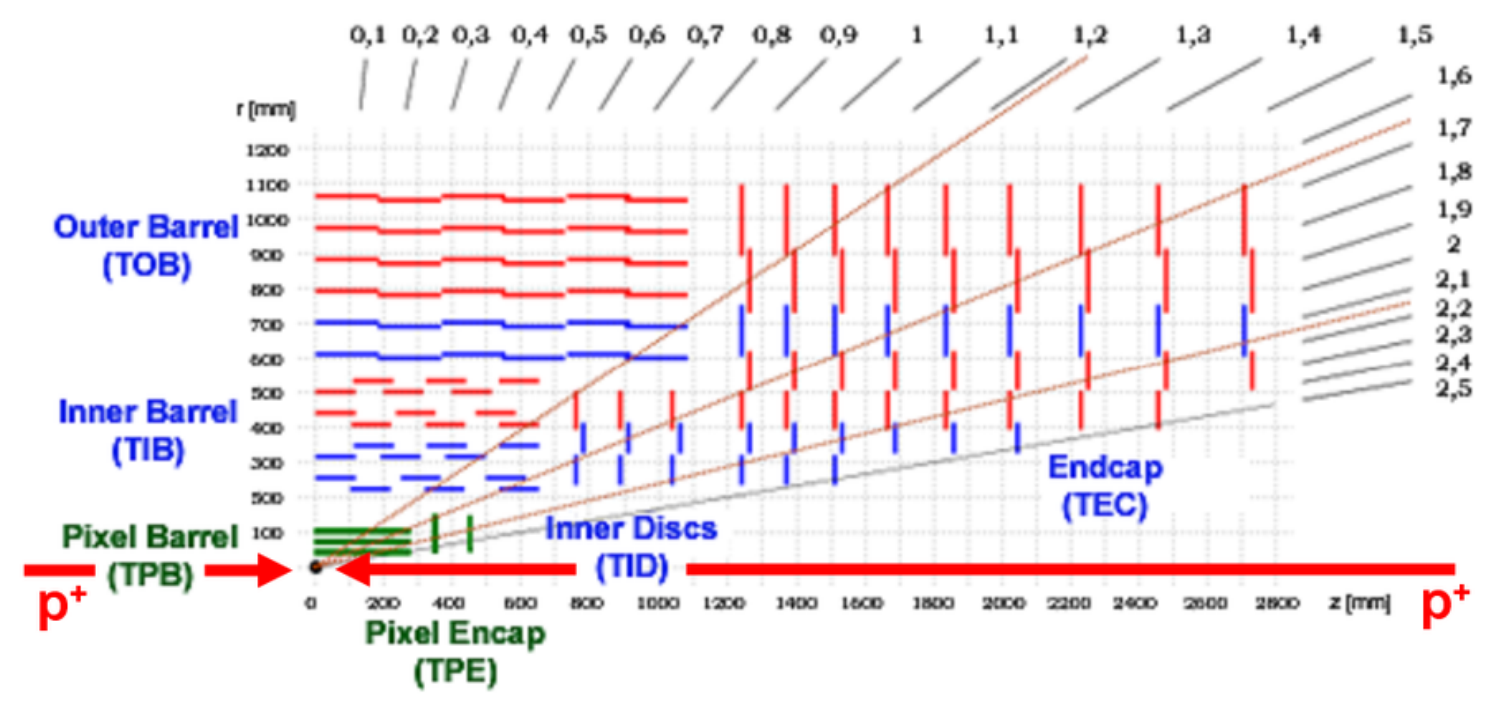

Figure 3.9: Layout of the CMS tracker: silicon pixel detector consisting of Tracker Pixel Barrel (TPB) and Tracker Pixel Endcap (TPE), silicon strip detector consisting of Tracker Inner Barrel (TIB), Tracker Inner Disks (TID), Tracker Outer Barrel (TOB), and Tracker EndCap (TEC).

The silicon pixel detector (Fig. 3.9) consists of three cylindrical barrel layers at a radius of $4.4,7.3$, and $10.2 \mathrm{~cm}$ from the beam line and two endcap disks on each side at $|z|=34.5$ and $46.5 \mathrm{~cm}$ with pixel cell size of $100 \times 150 \mu \mathrm{m}^{2}$. The detector provides a position resolution of about $10 \mu \mathrm{m}$ in $\mathrm{r}-\phi$ plane and about $20-40 \mu \mathrm{m}$ along the $\mathrm{z}$ axis.

The silicon strip detector surrounds the silicon pixel detector. It consists of four parts: the Tracker Inner Barrel (TIB), the Tracker Inner Disks (TID), the Tracker Outer Barrel (TOB), and the Tracker Endcap (TEC). The TIB has four barrel layers that cover the volume within $20<\mathrm{r}<55 \mathrm{~cm}$ and $|z|<65 \mathrm{~cm}$, the TID has three disks on each side that cover the region of $20<\mathrm{r}<55 \mathrm{~cm}$ and $65<|z|<118$ cm. TIB and TID provide a position resolution in $r \phi$ of $13-38 \mu \mathrm{m}$. The TOB covers volume within $55<\mathrm{r}<116 \mathrm{~cm}$ and $|z|<118 \mathrm{~cm}$, and has six barrel layers. The 

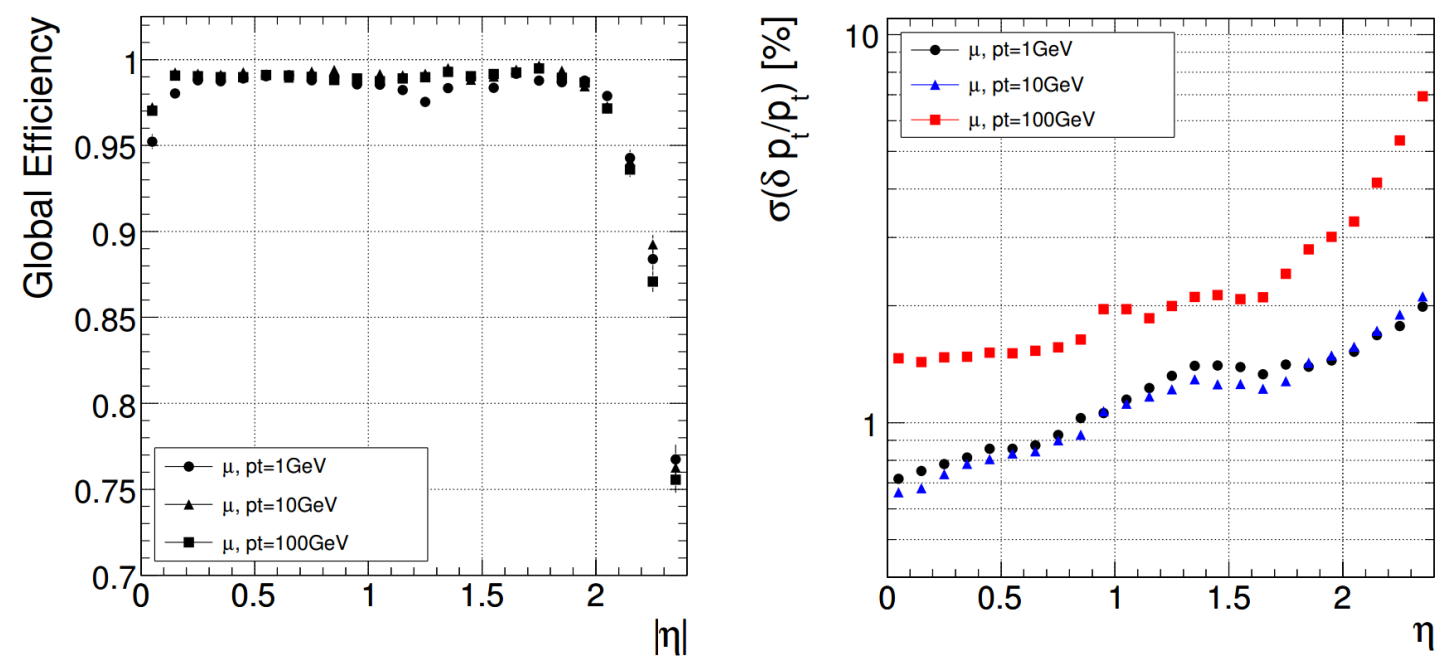

Figure 3.10: The performance of the CMS tracking system for muons with transverse momentum of 1,10 , and $100 \mathrm{GeV}$ : track reconstruction efficiency (left) and track transverse momentum resolution (right) as a function of pseudorapidity (from [22]).

TEC covers volume within $22.5<\mathrm{r}<113.5 \mathrm{~cm}$ and $124<|z|<282 \mathrm{~cm}$, and has nine disks on each side, with from four to seven concentric rings in each disk. TOB and TEC provide a position resolution in $r \phi$ of $18-47 \mu \mathrm{m}$. The inner part of the silicon strip detector has $320 \mu \mathrm{m}$ thick silicon sensors, the thickness of the sensors in the outer part is $500 \mu \mathrm{m}$. In the endcaps radial strips have pitch (the distance between strips) varying from 81 to $205 \mu \mathrm{m}$, and in the barrel silicon strips are parallel to the beamline and the pitch between them varies from 80 to $183 \mu \mathrm{m}$, .

The CMS tracking system provides robust and efficient reconstruction of trajectories of charged particles and precise measurement of their momenta. The Fig. 3.10 demonstrates the performance of the tracking system for muons with different transverse momenta: the reconstruction efficiency is about $98-99 \%$ over the most of pseudorapidity range, the transverse momentum resolution ranges from $0.7 \%$ to $2 \%$ for muons with transverse momentum of $10 \mathrm{GeV}$ and from $1.5 \%$ to $3 \%$ for muons with 


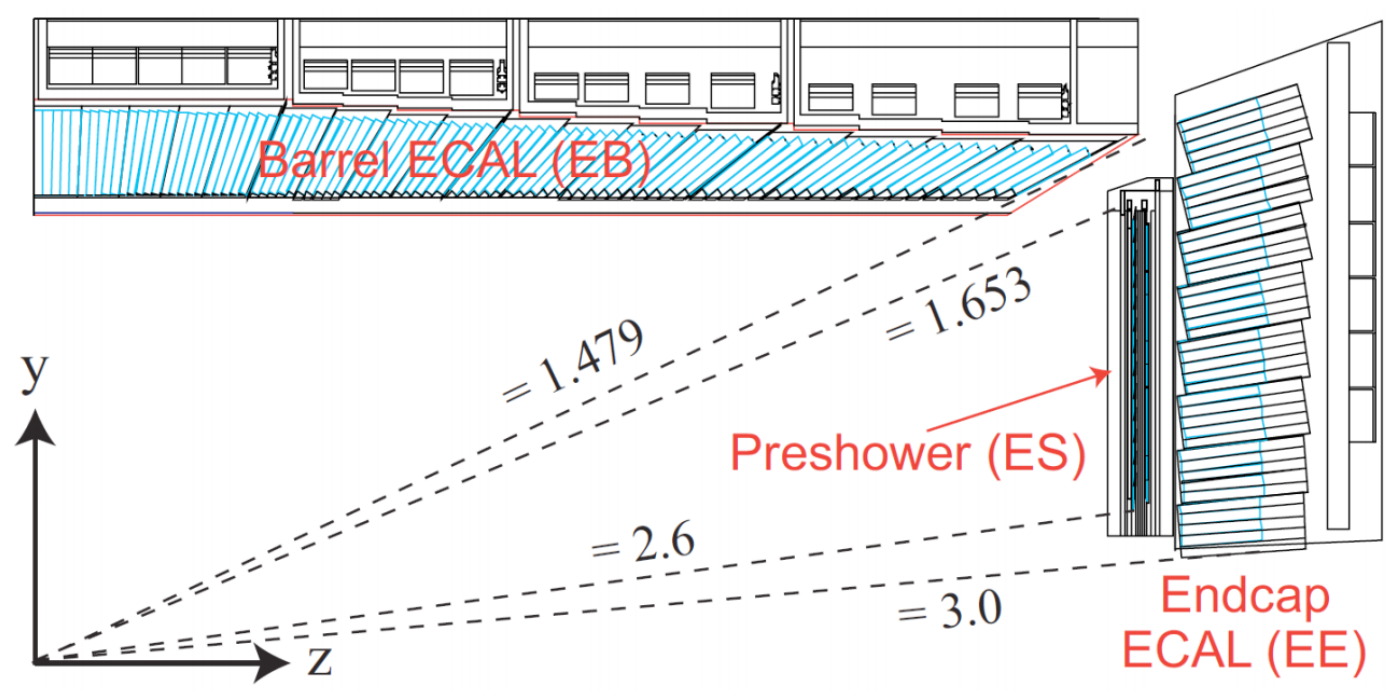

Figure 3.11: Longitudinal view of a quarter of the CMS electromagnetic calorimeter (ECAL): the Barrel ECAL (EB), the Preshower (ES) in front of the Endcap ECAL $(\mathrm{EE})$.

transverse momentum of $100 \mathrm{GeV}$ and pseudorapidity $|\eta|<2.5$. The resolution of transverse and longitudinal impact parameters improves from 90 and $150 \mu \mathrm{m}$ at $p_{T}=1 \mathrm{GeV}$ to 25 and $45 \mu \mathrm{m}$ at $p_{T}=10 \mathrm{GeV}$.

\subsubsection{Electromagnetic Calorimeter}

The CMS electromagnetic calorimeter (ECAL) is used to measure energy of electrons and photons. It is a nearly hermetic homogeneous calorimeter that surrounds the CMS tracking system [22]. It is made of 75848 lead tungstate $\left(\mathrm{PbWO}_{4}\right)$ scintillating crystals (61200 in the barrel and 7324 in the endcap regions). The $\mathrm{PbWO}_{4}$ crystals have been chosen due to their tolerance to high levels of radiation, the high density of $8.28 \mathrm{~g} / \mathrm{cm}^{3}$ and, thus, a short radiation length $X_{0}$ of $0.89 \mathrm{~cm}$ allows a compact detector. Small Moliére radius of $2.2 \mathrm{~cm}$ allows electromagnetic showers to be compact in $r-\phi$ plane. Finally, short scintillation decay time (about $40 \%$ of light 
is emitted within $5 \mathrm{~ns}$, about $60 \%$ in $15 \mathrm{~ns}$, about $80 \%$ in $25 \mathrm{~ns}$ ) allows ECAL to have a fast response, which is important for identification of out of time electrons and photons in a high pile-up environment.

The general layout of the ECAL is shown in Fig. 3.11. The Barrel ECAL (EB) covers pseudorapidity range of $\eta<1.479$ and consists of 36 supermodules. Each module consists of $1700 \mathrm{PbWO}_{4}$ crystals that have shape of truncated pyramids with the front face of $22 \times 22 \mathrm{~cm}^{2}$, rear face of $26 \times 26 \mathrm{~cm}^{2}$, and length of $23 \mathrm{~cm}$ corresponding to $25.8 X_{0}$. The avalanche photodiodes (APDs) are used to collect the scintillation light in the barrel crystals, they have been designed specially for the CMS ECAL. The Endcap ECAL (EE) covers pseudorapidity range of $1.48<|\eta|<$ 3.0. Each of two endcaps is divided into two halves (Dees). Each Dee consists of 3662 crystals with front face of $28.6 \times 28.6 \mathrm{~cm}^{2}$, rear face of $30 \times 30 \mathrm{~cm}^{2}$, and length of $22 \mathrm{~cm}$ corresponding to $24.7 X_{0}$. The vacuum phototriodes (VPTs) are used to collect the light emitted in the endcap crystals.

The ECAL Preshower (ES) is a sampling calorimeter placed in front of the Endcap ECAL. The main purpose of this preshower is to help with disambiguating photons and electrons from $\pi^{0}$ s. It covers pseudorapidity range of $1.653<|\eta|<2.6$. The preshower has two lead radiators that initiate electromagnetic showers of photons and electrons. Behind each of two radiators there is a layer with silicon strip sensors, strips in these layers are orthogonal to each other.

The ECAL energy resolution can be parameterized with the following stochastic, noise, and constant terms:

$$
\frac{\sigma(E)}{E}=\frac{2.8 \%}{\sqrt{E}} \oplus \frac{0.12}{E} \oplus 0.3 \%
$$

For electrons with energy higher than $15 \mathrm{GeV}$ the energy resolution is better 


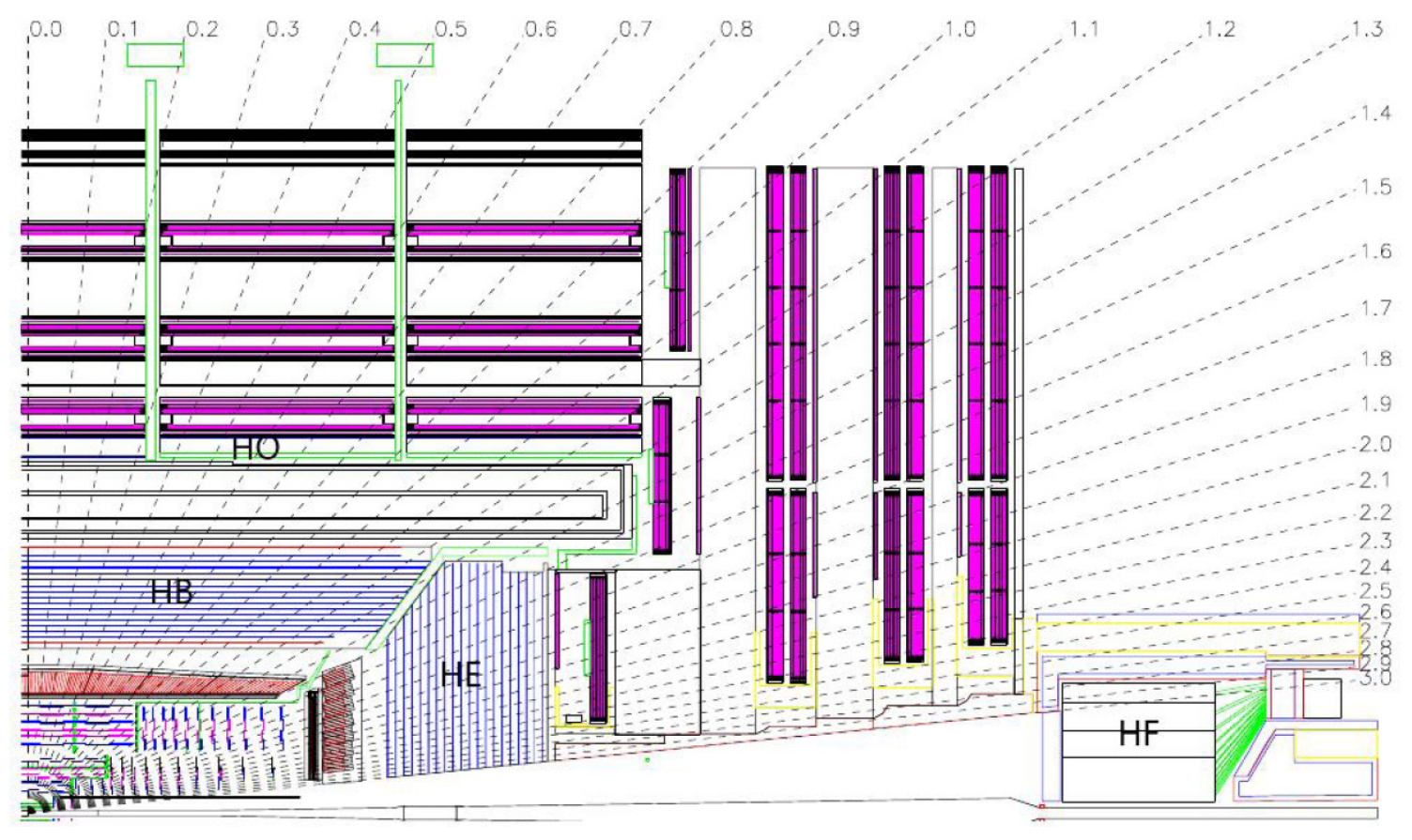

Figure 3.12: Longitudinal view of a quarter of the CMS hadronic calorimeter (HCAL): Barrel (HB), Endcap (HE), Outer (HO), and Forward (HF) hadronic calorimeters.

than $1 \%$, for electrons with energy of $40 \mathrm{GeV}$ the energy resolution reaches $0.6 \%$. For electrons with energy of $35 \mathrm{GeV}$ the position resolution is about $10^{-3}$ units in $\eta$ and $1.6 \mathrm{mrad}$ in $\phi$ (this corresponds to about $2 \mathrm{~mm}$ in $r \phi$ and $z$ directions).

\subsubsection{Hadron Calorimeter}

The CMS hadron calorimeter (HCAL) is used to measure energy of hadronic showers [22]. It surrounds the ECAL and completes the calorimeter system of the CMS. In addition, the HCAL plays an important role in measurement of neutrinos or other exotic particles that escape detection in CMS subdetectors by providing together with the ECAL an estimate of the missing transverse energy (MET) measured as an imbalance of energy in transverse direction. 
Fig. 3.12 shows the layout of the detector. The HCAL Barrel (HB) and two HCAL Endcaps (HE) are located between the ECAL and the solenoid (between 1.7 and $2.95 \mathrm{~m}$ from the beam line) and cover the pseudorapidity range up to $|\eta|=3.0$. The HB is complemented by the Outer HCAL (HO) placed just outside the solenoid to ensure a more complete containment of hadronic showers that start late and, thus, a better measurement of their energy. In addition, the Forward HCAL (HF) extends the pseudorapidity coverage of the HE by $3.0<|\eta|<5.0$ range. These parts of the HCAL are sampling calorimeters with alternating layers of the absorber that initiate hadronic showers and layers of active media to detect these showers.

The HB consists of 36 azimuthal wedges. Each wedge consists of 14 absorber layers made from brass ( 8 with thickness of $50.5 \mathrm{~mm}$ and 6 with thickness of 56.5 $\mathrm{mm}$ ), tiles of plastic scintillator acting as an active media, and two stainless steel plates in the front and in the back to provide a structural strength to wedges. The emitted light is collected by wavelength-shifting (WLS) fibres. The HB provides granularity of $(\Delta \eta \times \Delta \phi)=(0.087 \times 0.087)$.

The endcap calorimeter (HE) covers the region $1.3<|\eta|<3$ and consists of 19 layers of absorber brass plates with thickness of $78 \mathrm{~mm}$ and scintillator tiles with thickness of $9 \mathrm{~mm}$ providing granularity of $(\Delta \eta \times \Delta \phi)=(0.087 \times 0.087)$ and $(\Delta \eta \mathrm{x}$ $\Delta \phi)=(0.17 \times 0.17)$ in pseudorapidity ranges of $1.3<|\eta|<1.6$ and $1.6<|\eta|<3$, respectively.

The outer calorimeter $(\mathrm{HO})$ is placed outside the solenoid coil in front of the muon system and between its wheels and covers the pseudorapidity range up to $|\eta|=1.3$. The solenoid coil itself is used as an absorber for the HO. It provides granularity of $(\Delta \eta \times \Delta \phi)=(0.087 \times 0.087)$.

The forward calorimeter $(\mathrm{HF})$ is placed along the $\mathrm{z}$ axis $11.2 \mathrm{~m}$ away from the nominal interaction point and covers the pseudorapidity range of $3<|\eta|<5$. It had 


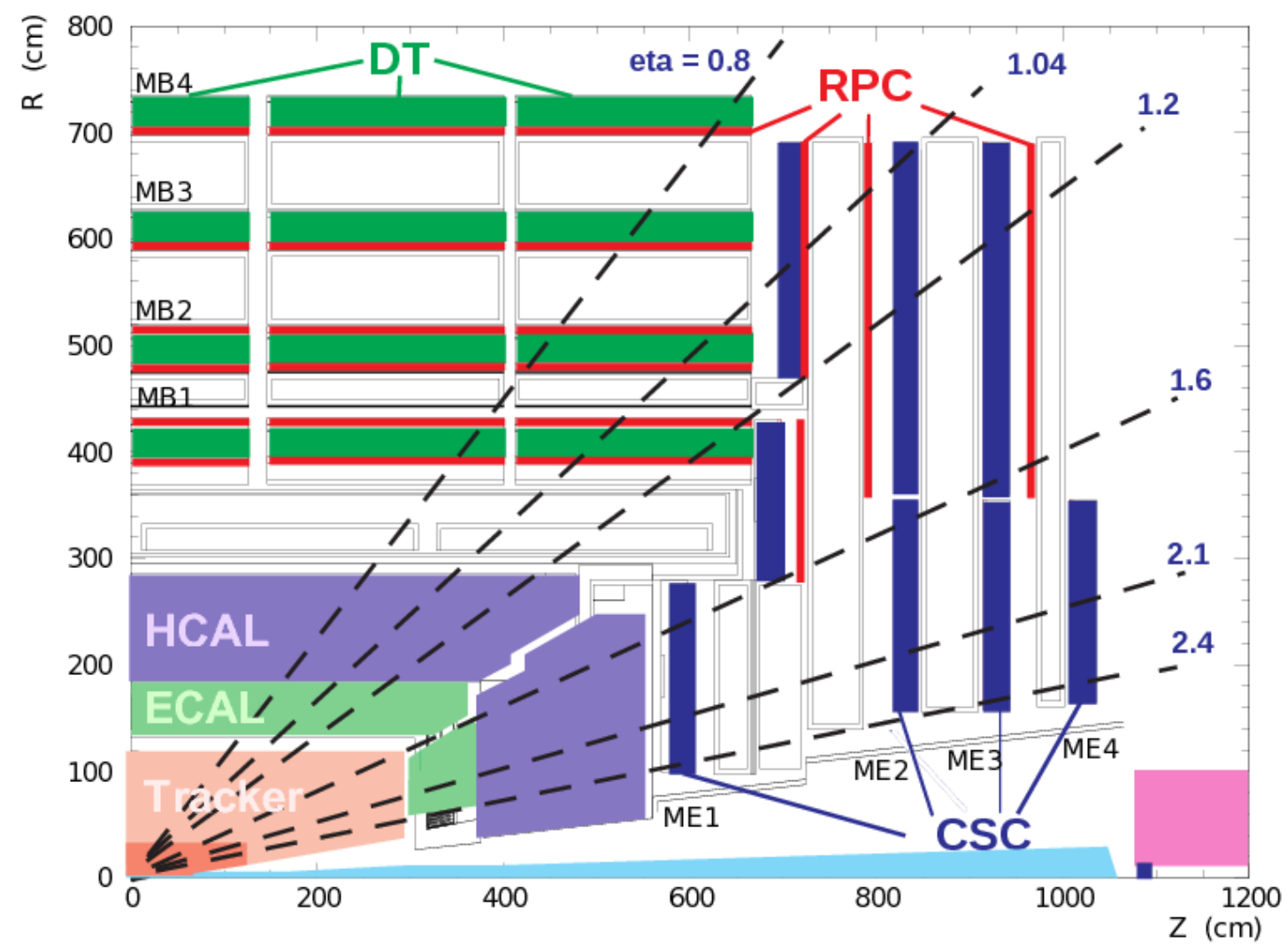

Figure 3.13: Longitudinal view of a quarter of the CMS detector illustrating the layout of the Muon System: Drift Tube chambers (DT), Cathode Strip Chambers (CSC), and Resistive Plate Chambers (RPC).

significantly higher particle flux passing through it than in other parts of the HCAL that poses an additional radiation hardness challenge. The HF consists of stainless steel absorbers and radiation hard quartz fibers that collect Cherenkov light.

\subsubsection{Muon System}

The CMS muon system provides robust and efficient reconstruction and identification of muons and, together with the tracker, it provides measurement of muon momentum with $1-10 \%$ precision within a wide kinematic range (Fig. 3.15). It plays an important role in the setup of the CMS trigger system [22]. 


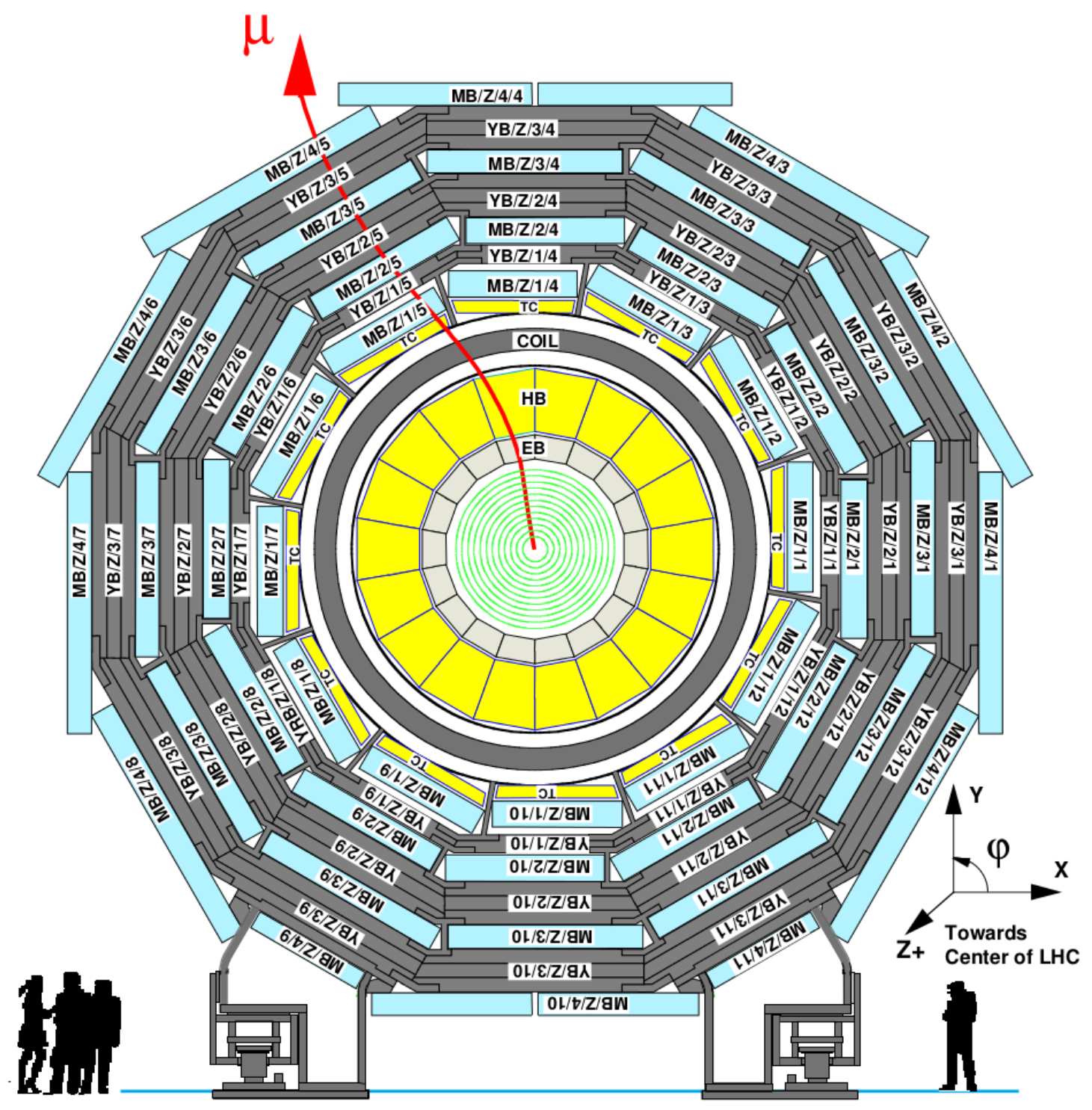

Figure 3.14: Transverse view of one of the five wheels with Drift Tube chambers in the barrel region of the CMS.

The muon system is placed in the outermost part of the CMS as muons lose very little of their energy in the tracker and both calorimeters, while other particles rarely reach muon detectors, thus providing a natural discrimination against non- 
muon backgrounds. Fig. 3.13 illustrates the layout of the muon system: it consists of the barrel region and two endcap regions covering the pseudorapidity range up to $|\eta|<2.4$. Three types of gaseous detectors are used in the muon system: Drift Tube chambers (DT), Cathode Strip Chambers (CSC) and Resistive Plate Chambers (RPC).
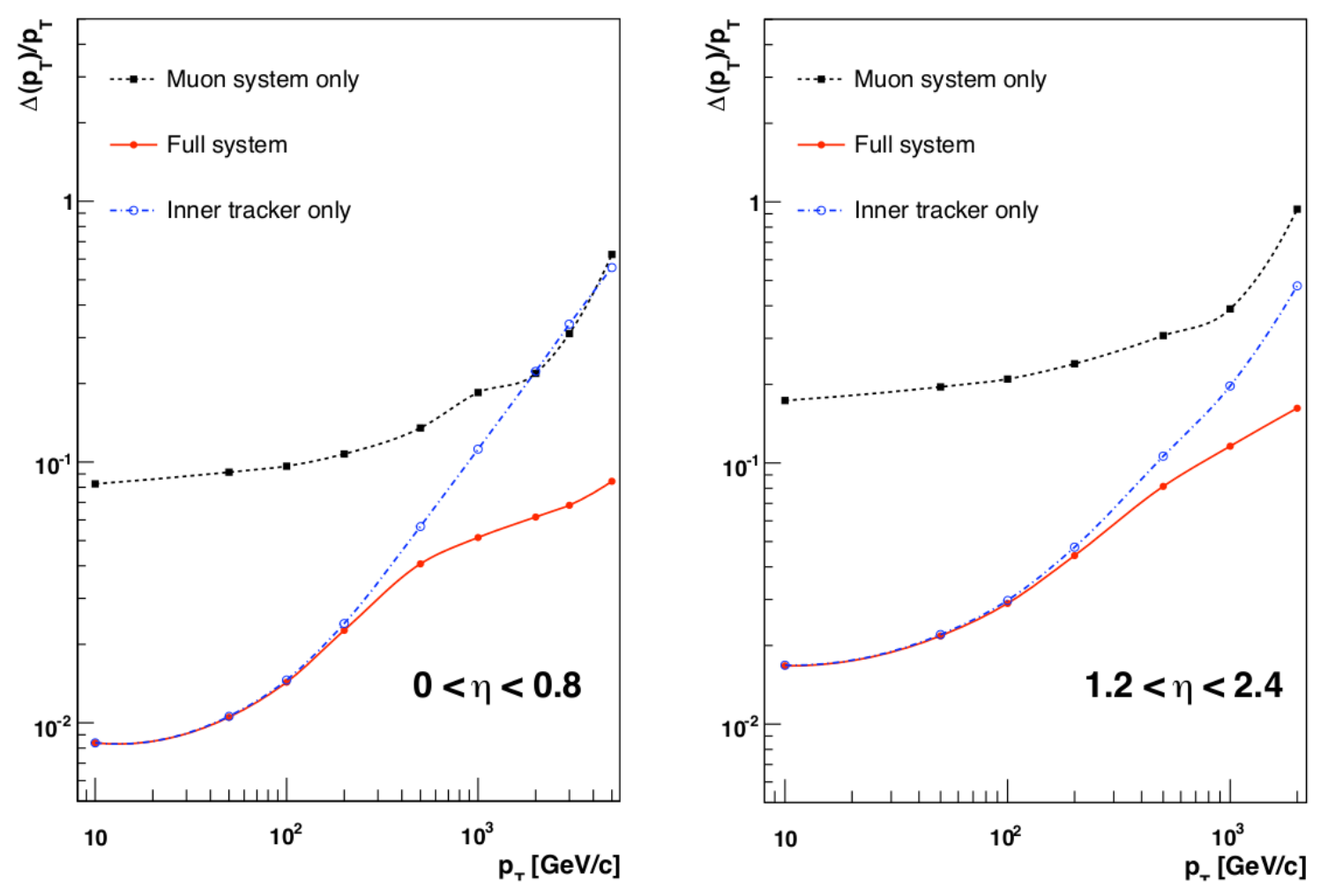

Figure 3.15: Muon transverse momentum resolution in pseudorapidity ranges of $|\eta|<0.8$ (left) and $1.2<|\eta|<2.4$ (right) using information from the tracker and the muon system separately or in combination (from [22]).

The Drift Tube (DT) technology has been chosen in the barrel region that covers the pseudorapidity range up to $|\eta|<1.2$ and where the flux of muons is not as high as in the endcap region and the magnetic field contained in the iron return yoke is 
almost uniform. The muon system in the barrel consists of four cylindrical stations around the beamline divided into wheels along the $\mathrm{z}$ axis. Fig. 3.14 illustrates the layout of one of the five wheels. Each of the first three stations has 60 chambers, and the last station has 70 chambers. Each DT chamber contains 3 superlayers (SL), where each superlayer consists of four layers of rectangular drift cells. A muon passing through a given drift cell initiates an avalanche of electrons, the measured drift time of these electrons determines the position of the muon in that drift cell. The two outer superlayers measure muon position along the $r-\phi$ direction, and the superlayer in the middle measures the position along the $\mathrm{z}$ axis (the last station does not have the middle superlayer).

The Cathode Strip Chambers (CSC) are used in the endcap region that covers the pseudorapidity range of $0.9<|\eta|<2.4$ with higher flux of muons and non-uniform magnetic field. The muon system in the endcaps consists of 540 chambers grouped into four stations along the $\mathrm{z}$ axis and three concentric rings around the beamline. Each chamber has a trapezoidal shape and contains six plates with cathode strips, the gaps between them are filled with gas and contain planes with anode wires that run along the $r-\phi$ direction. Cathode strips and anode wires measure $\phi$ and $\mathrm{r}$ coordinates, correspondingly.

The Resistive Plate Chambers (RPC) are double-gap gaseous chambers that complement DT chambers and CSCs in the pseudorapidity range up to $|\eta|<1.6$. There are six layers of RPCs in the barrel region and three layers in the endcap. Their spatial resolution is not as precise as of the DT or CSC detectors, but they have an excellent time resolution due to a fast response that is much shorter than the time between two consecutive bunch crossings.

For muons with transverse momentum less than $100 \mathrm{GeV}$, the transverse momentum resolution is driven by the tracker measurements, for muons with high transverse 


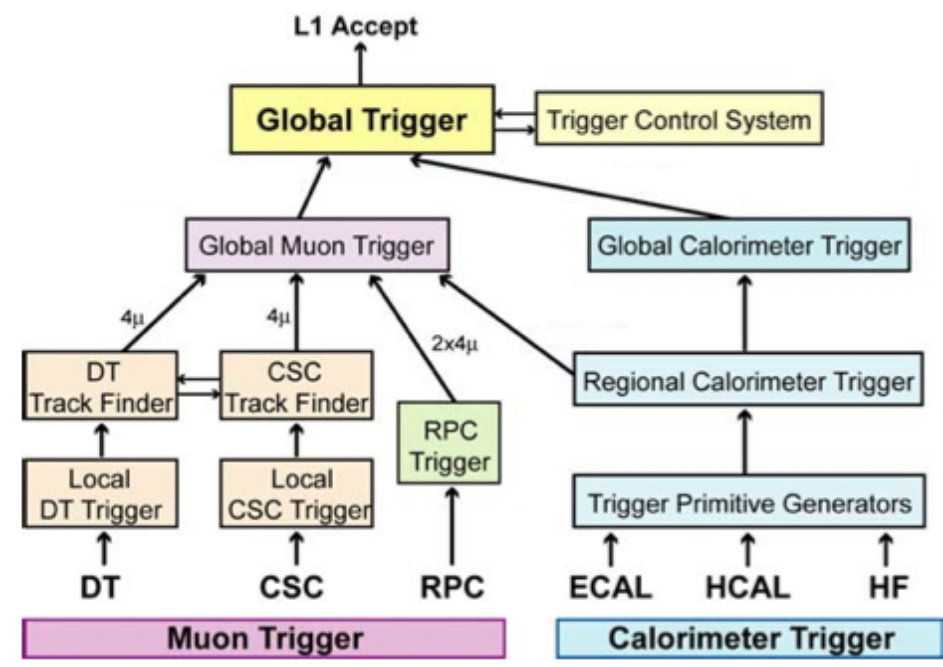

Figure 3.16: Overview of the CMS Level 1 (L1) Trigger (from [22]).

momentum the transverse momentum resolution provided by the tracker is greatly improved with addition of information from the muon system (Fig. 3.15).

\subsubsection{Trigger System}

At the nominal LHC luminosity of $10^{34} \mathrm{~cm}^{-2} \mathrm{~s}^{-1}$, one expects on average about 20 simultaneous proton collisions every proton bunch crossing. The design datasize describing one bunch crossing (raw signals from CMS subdetectors and derived quantities) is about $1 \mathrm{MB}$ [122]. Due to the technical and computational limitations of present day technology, it is not feasible to readout, process, and store the data for every collision.

The CMS trigger system selects the most interesting events (data about all collisions in one bunch crossing) and reduces the output rate from $40 \mathrm{MHz}$ down to $\sim 100 \mathrm{~Hz}$. This rate is reduced in two steps: the Level 1 (L1) trigger, and the High Level Trigger (HLT). The L1 trigger uses a custom-designed electronics, the HLT is a software system running on a farm of about 1000 CPUs. 
The Level 1 trigger uses data read out by the front-end electronics in the calorimeter and muon systems to select potentially interesting events and reduce the event rate from $40 \mathrm{MHz}$ down to about $100 \mathrm{kHz}$. A functional diagram of the $\mathrm{L} 1$ trigger is shown in Fig. 3.16: the L1 Global Trigger combines information from the L1 Global Calorimeter Trigger and the L1 Global Muon trigger that combines information from local muon triggers corresponding to three muon subsystems.

The LHT further reduces the event rate from $100 \mathrm{kHz}$ to about $100 \mathrm{~Hz}$. The HLT includes reconstruction and filtering algorithms arranged into paths, where each path targets events of a particular final state. For example, trigger paths for processes with muons may require the given event to contain a muon or two muons with transverse momenta higher than some predefined thresholds (single and double muon triggers). 


\section{EVENT RECONSTRUCTION}

Data collected by the CMS detector and selected by the trigger system is processed in offline in the CMS software (CMSSW). The algorithms implemented in the software are used to reconstruct abstract detector objects (particle tracks, energy clusters in calorimeters, etc.) that are then used to reconstruct objects correspond-

ing to physical particles (electrons, muons, photons, etc.) or groups of them (jets). Finally, these reconstructed objects are used in analyses of CMS data.

In addition to reconstruction and identification algorithms of specific particles in individual CMS subdetectors, CMS has adopted the Particle Flow (PF) algorithm $[123,124]$ that provides a framework for consistent use of the information from several subdetectors allowing more optimal discrimination between particles.

The PF algorithm reconstructs all stable particles in the event: photons, electrons, muons, charged and neutral hadrons, and uses them to reconstruct jets, to measure missing transverse energy and provide an estimate of direction and energy of invisible particles, etc. The algorithm starts with reconstruction of its fundamental PF "elements": charged-particle tracks and the calorimetric clusters, that are further topologically linked into PF "blocks", and then these PF blocks are interpreted in terms of particles.

This chapter will briefly review reconstruction of particles of different species within the CMSSW.

\subsection{Track Reconstruction}

Reconstruction of tracks of charged particles starts with local reconstruction reconstruction of pixel and strip hits via clustering of signals above certain thresholds in pixel and strip channels and finding positions and uncertainties of these clusters. 
Further, these hits are used by Combinatorial Track Finder (CTF) [125] to estimate the momentum and position of charged particles. The tracks are reconstructed in multiple iterations of CTF, which together form a so-called iterative tracking algorithm used by CMS. The initial iterations are focused on tracks that are the easiest to reconstruct. Hits of already reconstructed tracks are not considered in the subsequent iterations, reducing their combinatorial complexity. There are six iterations: the first considers tracks with $p_{\mathrm{T}}>0.8 \mathrm{GeV}$ and with at least three pixel hits in vicinity of the proton-proton interaction point. The second iteration reconstructs tracks with two pixel hits and all subsequent iterations are used for reconstruction of tracks due to charged particles produced away from the beam spot and any tracks that haven't been reconstructed in previous iterations.

Each iteration of CTF includes the following four steps:

- Seed generation: reconstruction of initial track candidates using 2 or 3 hits;

- Track finding: extrapolation of seed candidates along the expected trajectory and addition of any associated hits to the track;

- Track fitting: estimation of track parameters using Kalman "filter" and "smoother" $[125]$

- Track selection: setting quality flags and elimination of tracks that do not pass set quality requirements.

The performance of track reconstruction for muons in $Z \rightarrow \mu \mu$ decays is measured using the so-called "tag-and-probe" technique [126] (see Fig. 4.1(left)). The measured tracking efficiency is better than $99 \%$ both in data and simulation, the inefficiencies are associated with non-instrumental regions near the boundaries of the tracker components. 

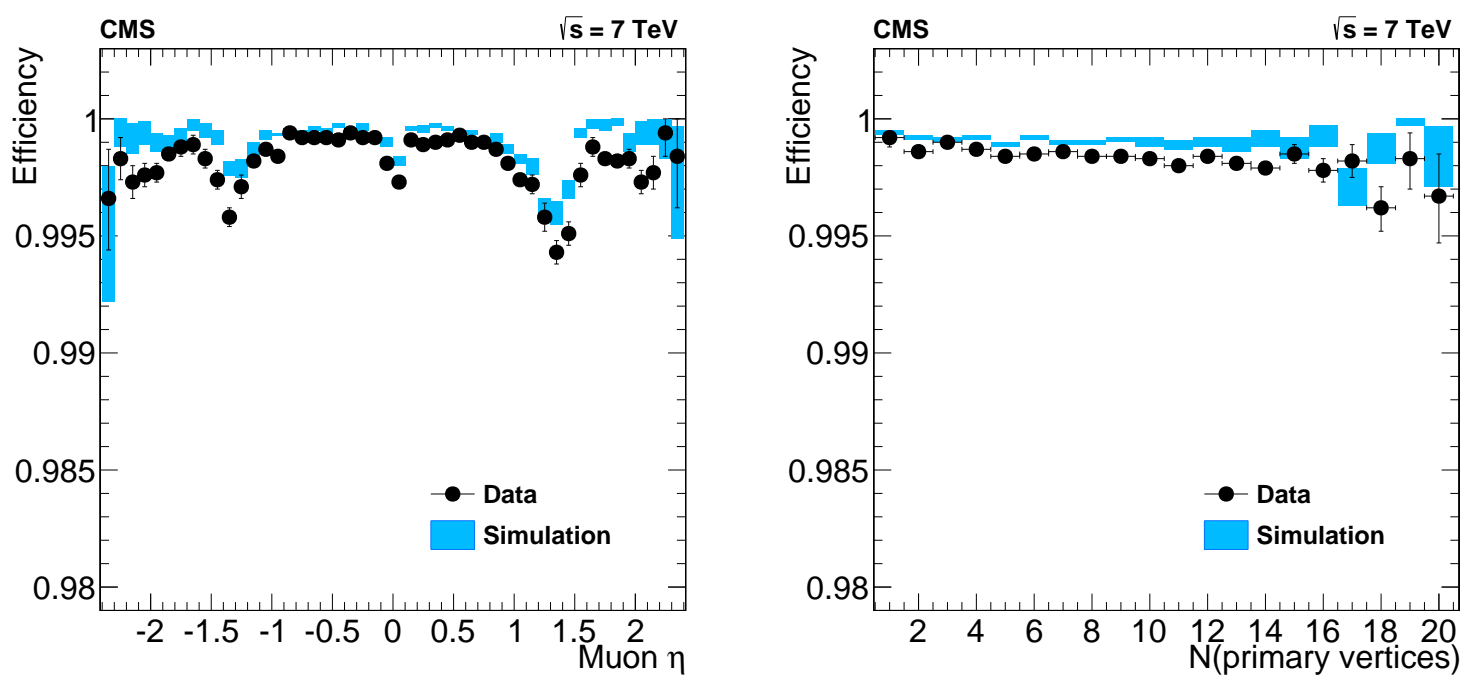

Figure 4.1: Tracking efficiency measured with a tag-and-probe technique for muons from $\mathrm{Z}$ decays as a function of the muon $\eta$ (left) and the number of reconstructed primary vertices in the event (right) for data (black dots) and simulation (blue bands) (from [121]).

\subsection{Primary Vertex Reconstruction}

Interaction point reconstruction procedure estimates positions and their uncertainties for all proton-proton collisions in each event including the primary vertex and vertices from pile-up collisions. The procedure includes the following three steps:

- Track selection: selection of tracks consistent with being produced promptly by introduction of a requirement on the maximum value of significance of the transverse impact parameter with respect to the beam spot;

- Track clustering: combining selected tracks into clusters of tracks that appear to originate from the same interaction point using a deterministic annealing (DA) algorithm [127];

- Track fitting: estimation of vertex position and its uncertainty by fitting tracks 

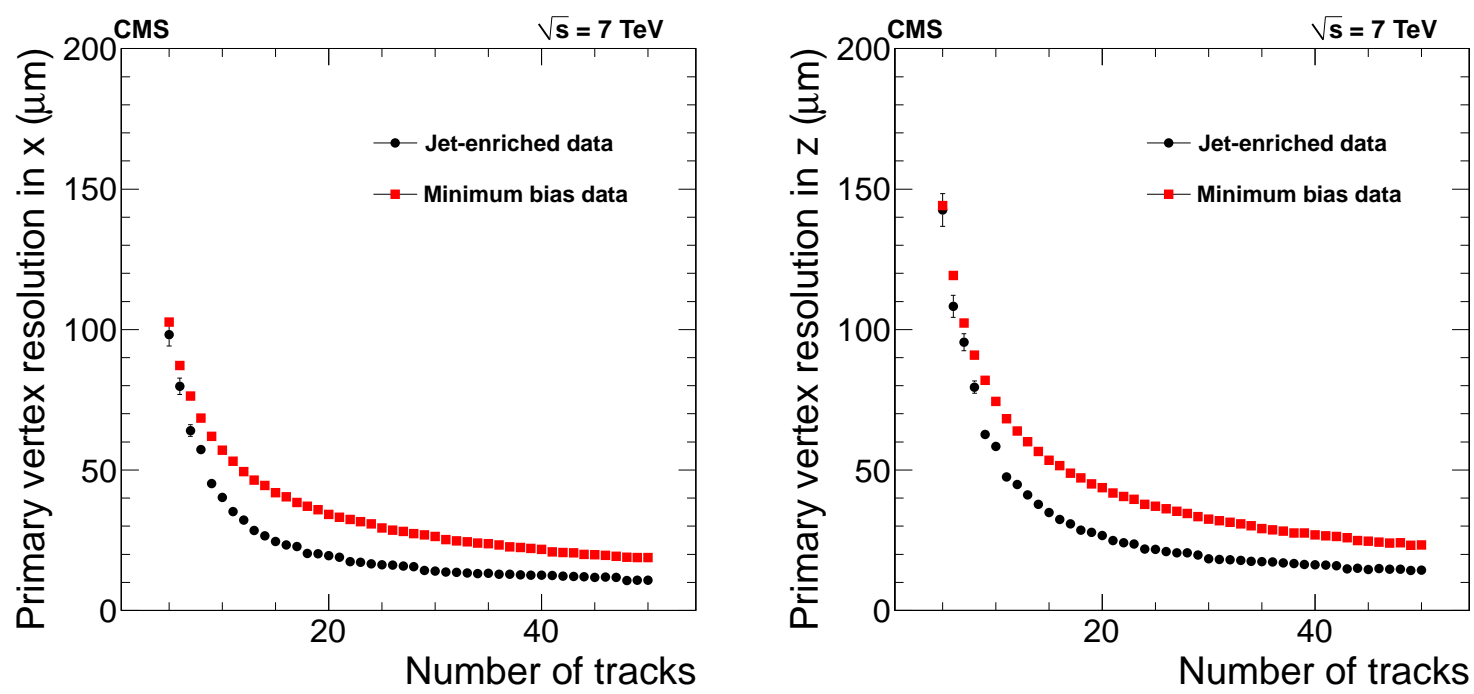

Figure 4.2: Primary-vertex resolution in $\mathrm{x}$ (left) and in $\mathrm{z}$ (right) as a function of the number of tracks at the fitted vertex (from [121]).

associated with it using an adaptive vertex fitter [128].

The resolution in the primary vertex position strongly depends on the number of tracks used to fit the vertex (see Fig. 4.2). The primary vertex reconstruction efficiency is close to $100 \%$ when more than two tracks are used for its reconstruction (see Fig. 4.3).

\subsection{Particle Identification}

\subsubsection{Electrons}

Reconstruction of electrons [129] uses two seeding algorithms that complement each other: tracker driven and calorimeter driven. The former one is designed for low $p_{T}$ electrons and electrons in jets, while the latter is optimized for higher $p_{T}$ and isolated electrons.

ECAL driven seeding is based on reconstruction of ECAL superclusters (groups of one or more ECAL clusters of energy deposits [130]). ECAL superclusters are 


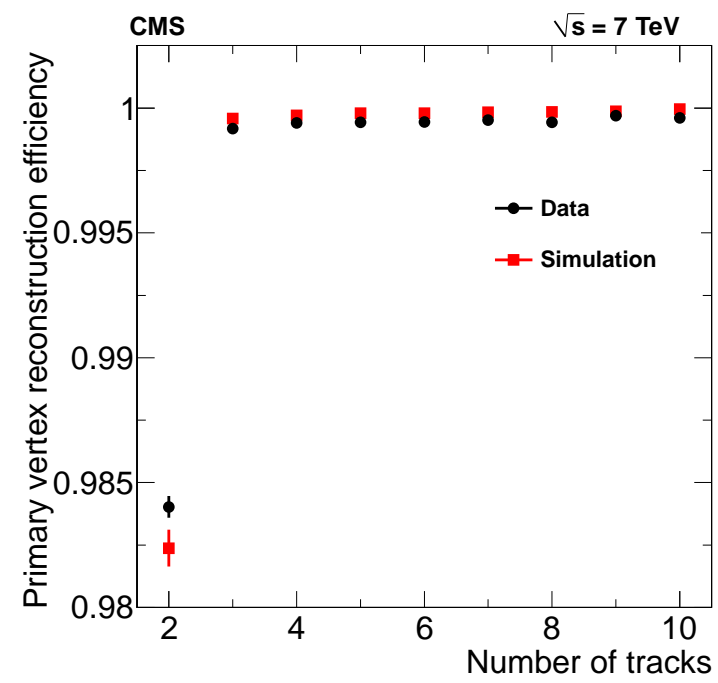

Figure 4.3: Primary-vertex reconstruction efficiency as a function of the number of tracks in a cluster, measured in minimum-bias data and in $\mathrm{MC}$ simulation (from $[121])$.

reconstructed using Hybrid (barrel electrons) and Island algorithms (endcap electrons) [131] that take into account their typical width in $\eta$ direction and typical spread in $\phi$ direction due to the bending of electrons in magnetic field and electron bremsstrahlung.

ECAL superclusters are then matched to track seeds (pairs or triplets of pixel tracker hits) and electron trajectories are built from track seeds using a Gaussian Sum Filter (GSF) [132].

In the PF algorithm, all tracks in PF blocks passing a dedicated electron preidentification stage are refit with GSF to follow their trajectories to the ECAL. Identification of PF electrons is performed with a combination of a number of tracking and calorimetric variables. Tracks and ECAL clusters associated with identified electrons are removed from the list of PF blocks to avoid double-counting of energy in the subsequent steps of event reconstruction. 


\subsubsection{Muons}

Muon reconstruction [126] starts with the local reconstruction in the muon system. Hits are identified in the detection layers of DT, CSC, and RPC chambers. DT and CSC segments (muon stubs) are built from linear fits of DT and CSC hit positions. These segments are used for seeding the muon trajectory building using a Kalman Filter [133]. The resulting muons are referred to as standalone muons.

Standalone muons can be then combined with tracks left by muons in the tracker using one of the following two approaches:

- Global muon reconstruction (outside-in): a standalone muon reconstructed in the muon system is matched to a track in the tracker, a global muon track is built combining the hits from the tracker and muon system.

- Tracker muon reconstruction (inside-out): all tracks reconstructed in the tracker with $p_{T}>0.5 \mathrm{GeV}$ and $\mathrm{p}>2.5 \mathrm{GeV}$ are considered as muon candidates. They are extrapolated to the muon system taking into account the expected energy loss and the uncertainties due to multiple scattering. If there is a match with at least one DT or CSC segment, the track is classified as a tracker muon track.

If momentum of a global muon is consistent with momentum of associated tracker track within three standard deviations, the muon gives rise to a PF muon, and the associated track is removed from PF blocks.

As it shown in Sec. 5.2.1.1, reconstruction of tracker muons is much less sensitive to the closeness of muons than global muon reconstruction. In the search presented in this dissertation, we expect muons in dimuons to be close-by, therefore, the muon candidates in the search are selected from the list of reconstructed tracker muons.

The muon system, together with the tracker, provides measurement of muon 
transverse momentum $p_{T}$ with $1-10 \%$ precision for muons with $p_{T}$ ranging from 10 GeV to $1 \mathrm{TeV}$ (see Fig. 3.15 in Sec. 3.2.5). The dimuon mass resolution, which is important for this study, is discussed in detail in Sec. 5.2.2.4.

\subsubsection{Charged and Neutral Hadrons, Photons}

Tracks that haven't been used in muon or electron reconstruction are then linked to the ECAL and HCAL clusters. In order to separate the neutral components (neutral hadrons and photons) and the charged particles in the PF block, a comparison between the momentum of the tracks and the calorimetric energy is performed. The comparison takes into account corrections for the non linearity of the calorimeter response and threshold effects (cluster calibration [123]).

In the case where the total calibrated cluster energy is smaller than the total charged particle momenta by more than three standard deviations, a search for muons passing loose identification criteria (global muons with momentum uncertainty less than $25 \%$ ) is performed. The remaining tracks are removed progressively ordered by their $p_{T}$ uncertainty. Tracks with an uncertainty higher than $1 \mathrm{GeV}$ are removed from the list until all such tracks are examined or the total track momentum reaches the calibrated cluster energy. Each remaining track gives rise to a charged hadron with a momentum taken from the tracker measurement and a mass corresponding to a $\pi^{ \pm}$.

The calibrated cluster energy can be occasionally reconstructed higher than the total charged particle momentum. If the difference exceeds one standard deviation of the cluster energy, the algorithm will create additional neutral particles. The preference in the ECAL is given to photons. This is justified by the observation than in jets $25 \%$ of the energy is carried by photons, while neutral hadrons leave only $3 \%$ of the jet energy in the ECAL. 
If the calorimetric excess is larger than the total ECAL cluster energy, the ECAL energy is associated with a photon and the remaining part of the excess is associated with a neutral hadron. Otherwise, the energy excess is associated with a photon. The remaining ECAL and HCAL clusters not linked to any track give rise to photons or neutral hadrons, respectively.

\subsubsection{Jets}

In this section only reconstruction of PF Jets is covered. PF Jets are reconstructed from the full set of PF particles. Among several jet reconstruction algorithms and methods used in CMS, the most commonly used technique is the anti- $k_{T}$ algorithm [134] with a distance parameter $\mathrm{R}=0.5$. The $\mathrm{PF}$ jet momentum and spacial resolution are improved with respect to the so called calo-jets reconstructed as clusters of ECAL and HCAL cells. This is due to the use of the combination of the tracker and the granularity of the ECAL to distinguish and precisely measure the energy of charged hadrons and photons, which together carry on average about $90 \%$ of the jet energy.

With information about reconstructed tracks and vertices available before jet clustering, contributions from pile-up (PU) proton-proton collisions to the jet energy can be reduced by up to $50 \%$ by removing charged particles originating from pile-up vertices [135]. In addition, a dedicated boosted decision tree (BDT) with 12 distinct variables have been trained to identify and reject pile-up jets [136].

CMS has adopted a factorized approach to jet energy corrections (JEC) [137]. The JEC factors are primarily derived from simulated events and small differences between data and simulation are corrected using data-driven methods. This results in better than $1 \%$ precision in the jet energy scale for jets above $100 \mathrm{GeV}$ in the central region of CMS. 


\subsubsection{Tau Leptons}

$\tau$ leptons decay either to leptons (muons or electrons) or hadronically; all tau decays contain one or more neutrino in the final state. The mean lifetime of $\tau$ leptons is $3 \cdot 10^{-13} \mathrm{~s}$, so only the decay products of $\tau$ leptons can be detected. The $\tau$ reconstruction algorithms usually refer to hadronically decaying $\tau$ leptons denoted as $\tau_{h}$, as electrons and muons from $\tau$ decays are not distinguishable from electrons or muons originating from proton-proton collisions. Almost all $\tau_{h}$ leptons decay into one or three charged hadrons, a tau neutrino, and sometimes one or more neutral pions that promptly decay to pairs of photons.

The $\tau$ lepton reconstruction algorithm used in most CMS analyses using $\tau$ leptons is the "hadron plus strips" (HPS) algorithm [138]. The algorithm starts reconstruction of a $\tau_{h}$ candidate from a PF jet reconstructed using anti- $k_{T}$ algorithm with a distance parameter $R=0.5$ [134]. Pairs of photons from decays of neutral pions may undergo conversions in the CMS tracker material that lead to broad calorimeter signatures in $\phi$ direction. This effect is taken into account in the HPS algorithm by reconstructing photons from PF photons or electrons in "strips" (windows of size $\Delta \eta=0.05$ and $\Delta \phi=0.2)$. Reconstructed strips are then combined with the charged hadrons to reconstruct individual $\tau_{h}$ decay modes mentioned above. It is assumed that all charged hadrons used in this combination are charged pions.

Finally, all reconstructed $\tau_{h}$ candidates are required to be isolated: apart from the $\tau_{h}$ decay products, there should be no other charged hadrons or photons present in a certain isolation cone around the direction of $\tau_{h}$. This requirement allows to significantly reduce the rate of jet $\rightarrow \tau_{h}$ misreconstruction. In addition, dedicated variables are calculated for discrimination against electrons and muons, in order to reduce rates of $e \rightarrow \tau_{h}$ and $\mu \rightarrow \tau_{h}$ misidentification. 


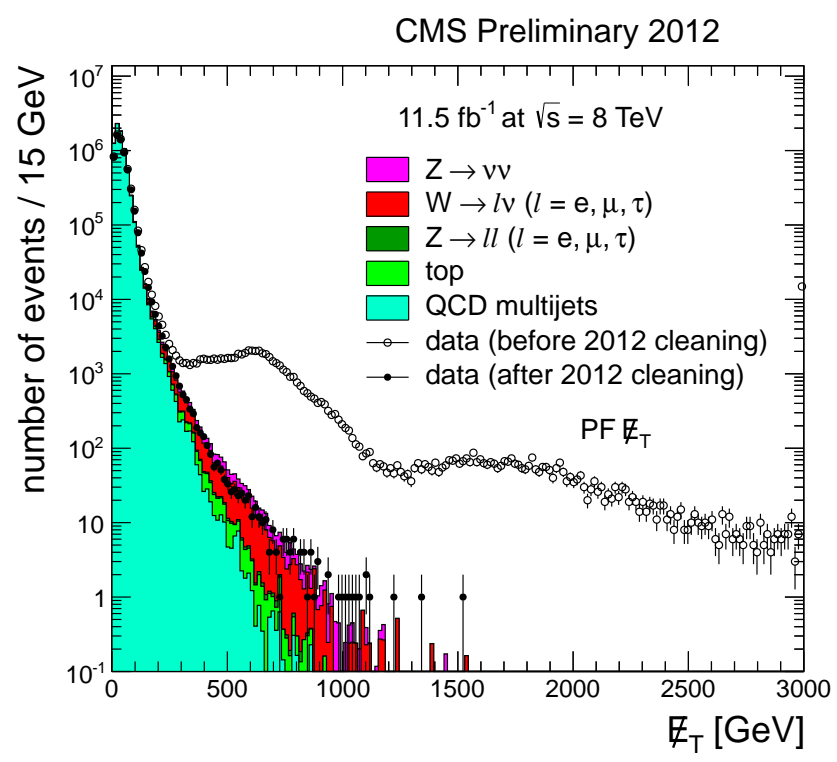

Figure 4.4: Distribution of PF MET for dijet events in data before (hollow markers) and after (solid markers) applying the cleaning algorithms compared to simulation (filled histograms) (from [139]).

\subsubsection{Missing Transverse Energy (MET)}

The CMS detector is designed to reconstruct stable particles produced in the proton-proton collisions. Particles that cannot be reconstructed are neutrinos, particles falling out of the detector acceptance, or hypothetical neutral weakly interacting particles, if they exist. As the longitudinal component of the momentum of the interacting partons during the collision is unknown, hadron collider experiments use missing transverse energy defined as the imbalance in the transverse momentum of all visible particles.

CMS has developed several algorithms to reconstruct MET, e.g. tracker based, calorimetric, depending on the objects used to compute it (particle candidates, 
calorimeter clusters, etc). The most widely used MET reconstruction algorithm is the PF MET algorithm that defines missing transverse energy as a vectorial sum of all the visible PF particles momenta. Fig. 4.4 illustrates a good agreement between PF MET in data and simulation. 


\section{DATA ANALYSIS *}

This chapter describes a search for the production of a non-SM Higgs boson denoted as $h$ decaying into a pair of new light bosons of the same mass denoted as $a$, each of which subsequently decays to a pair of oppositely charged muons (dimuon) ( $h \rightarrow 2 a+X \rightarrow 4 \mu+X$, where $X$ are possible additional particles from cascade decays of the Higgs boson). This experimental signature is motivated by models with hidden sectors discussed in Sec. 2.2.2.

Sec. 5.1 provides an overview of the search and the overall strategy. Sec. 5.2 describes analysis selections and provides details of their optimization. Sec. 5.3 summarizes efficiency of these selections for benchmark models (Sec. 2.2.2.2). Sec 5.4 explains procedure for estimation of contrbutions from background processes passing the analysis selections. Sec 5.5 summarizes systematic uncertainties and their sources. Sec 5.6 reviews analysis findings and their statistical interpretation as well as their application to benchmark models.

\subsection{Analysis Strategy and Overview}

One of the main technical challenges of this analysis is closeness of pairs of muons originating from the decays of $a$ 's that are typically highly boosted due to their light mass. There are several sets of selection criteria for offline muon candidates recommended for analyses of CMS data with muons in the final state. However, these recommended criteria have been shown to be not optimal for analyses with close-by muons in the final state. The strategy of this analysis is to design and optimize

\footnotetext{
*Part of this section is reprinted with permission from "Search for Light Resonances Decaying into Pairs of Muons as a Signal of New Physics" by S. Chatrchyan et al., 2011. JHEP 07098 , Copyright 2011 by CERN and "Search for a Non-Standard-Model Higgs Boson Decaying to a Pair of New Light Bosons in Four-Muon Final States" by S. Chatrchyan et al., 2013. Physics Letters B 726 564-586, Copyright 2013 by CERN
} 
an efficient selection of events and offline muon candidates, while maintaining low sensitivity of the analysis to kinematics of the final state particles. The latter is important to maintain independence of the results from details of specific theoretical benchmark scenario predicting this final state (model independence). Another important goal of the optimization is to maximize suppression of background events to achieve high sensitivity of the results to new physics.

The selection criteria are studied using fully reconstructed simulated events of the two benchmark models introduced in Sec. 2.2.2.2. Selection efficiency $\alpha_{R E C O}$ is defined as the fraction of signal events passing all selection requirements in a default simulation. As a reference, we also use a quantity $\alpha_{G E N}$, defined as the fraction of signal events passing requirements on the transverse momenta and pseudorapidities of simulated muons. Ratio of $\alpha_{R E C O}$ and $\alpha_{G E N}$ reflects the impact of detector and reconstruction related effects. To preserve model independence of the search, the ratio should be maintained as constant as possible in order to keep the contributions of detector-related inefficiencies independent of the details of a given model. Therefore, in order to achieve model independence of the analisys, the selection requirements are optimized to maintain constant ratio of $\alpha_{R E C O}$ and $\alpha_{G E N}$ across all benchmark samples.

The search uses events selected by a double muon trigger that requires at least two muons in each event, one with $p_{T}>17 \mathrm{GeV}$ and another one with $p_{T}>8$ $\mathrm{GeV}$. Selected events are required to contain pairs of muons, each consistent with being originated from a light boson (muons of opposite charge are combined into a dimuon if each muon has $p_{T}>8 \mathrm{GeV}$, invariant mass of the dimuon is sufficiently low $m_{\text {inv }}\left(\mu^{+} \mu^{-}\right)<5 \mathrm{GeV}$, and trajectories of muons in each dimuon are consistent with being originated from the same vertex). The dimuons are required to be compatible with being produced in the same proton-proton collision and to be isolated from the 
rest of the event activity.

Contributions from background processes passing analysis selections are found to be dominated by the $b \bar{b}$ events, where muon pairs are produced either via semileptonic decays or from decays of light resonances. A dimuon isolation requirement leads to a large suppression of $b \bar{b}$ contribution resulting in a nearly zero background final state.

Finally, reconstructed invariant masses of two dimuons are required to be consistent with each other within the detector resolution. On the two-dimensional distribution of their invariant masses $m_{1}$ and $m_{2}$ (Fig. 5.1(right)), the signal events would appear as a peak somewhere near the diagonal, while the background events would be scattered across the entire distribution. Known Standard Model resonances appear as vertical or horizontal lines of enhancements in events where one of the dimuons comes from a resonance. Background events with two SM resonances can also form a peak at the diagonal. However, as fragmentation of each of the two b-jets occurs independantly, the size of the enhancement at the diagonal is proportional to the product of probabilities of each b-jet yielding a specific resonance and is therefore known. This two-dimensional distribution is searched for an enhancement near the diagonal, which is then compared with the background expectation near the diagonal that is obtained by extrapolating data from the off-diagonal region of the distribution. The signal region is defined as a "corridor" near the diagonal: $\left|m_{1}-m_{2}\right|<0.13+0.065\left(m_{1}+m_{2}\right) / 2$, with the width corresponding to at least $5 \sigma$ in core detector resolution (see Sec. 5.2.2.4). The search is a "blind" analysis: all selection criteria are chosen without using data from the signal region. The distribution within the signal region is analyzed using a binned likelihood fit, where the signal shape is modeled using a Crystal Ball function and the background shape is determined from the background-enriched samples.

There is no constraint on the invariant mass of all four muons to preserve the 


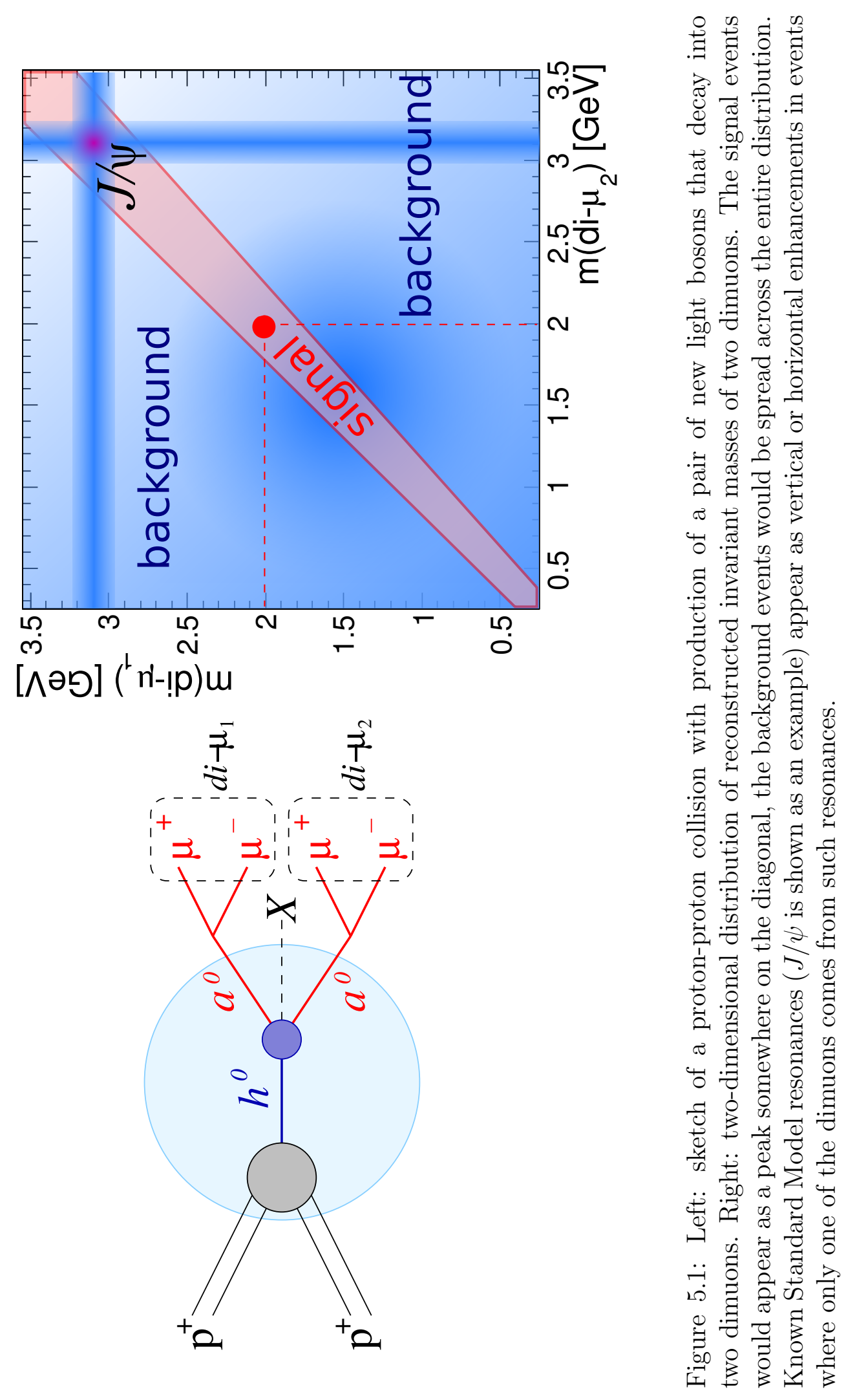


model independence of the analysis (such constraint could potentially enhance sensitivity of the search for $h \rightarrow 4 \mu$ as $m(4 \mu)=m_{h}$, but, for example, SUSY production of the light dark fermions in cascade decays yields events that have no such correlation).

\subsection{Analysis Selections}

All analysis selection requirements can be divided into two groups: selection of offline muon candidates and topological event selection. Both Sec. 5.2.1 and Sec. 5.2.2 start with overview and summary of each group and continue with details of optimization and justification of specific selection requirements.

\subsubsection{Offline Muon Candidate Selection}

In this analysis the offline muon candidates are required to be reconstructed as tracker muons (with the "inside-out" algorithm, see Sec. 4.3.2) as well as pass the following tracking related quality requirements:

- transverse momentum $p_{T}>8 \mathrm{GeV}$,

- pseudorapidity within $|\eta|<2.4$,

- number of valid hits in the silicon tracker $\geq 8$,

- normalized $\chi^{2}$ of track in the silicon tracker $<4$,

- number of matched muon segments $\geq 2$ (arbitrated: segments that form a better match with a different muon candidate are not considered).

\subsubsection{Choice of Muon Identification Algorithm}

The performance of muon reconstruction algorithms is affected by closeness or crossing of muon trajectories. The performance of the tracker muon (inside-out) and the global muon (outside-in) reconstruction algorithms has been compared in [140]. 
The comparison has shown that tracker muons are much less sensitive to the presence of close-by muons. The reason for it is that in case of the global muon reconstruction, the muon stand-alone track-finding gets confused by the presence of multiple close-by muon segments due to its limited pointing and momentum resolution.

Figure 5.2 shows a comparison of the performance of the tracker muon (outside-in) and the global muon (inside-out) muon reconstruction algorithms using Monte Carlo simulation. The plots show the probability of finding both muons in a simulated sample of dimuon events that are generated with a flat invariant mass distribution between two muon masses and $50 \mathrm{GeV}$. Reconstruction efficiency is shown as a function of the separation of their trajectories in the muon system.

The separation of muon trajectories is quantified on a surface of a cylinder with $600 \mathrm{~cm}$ radius and centered on the beamline (corresponds to the DT station 3 in the muon barrel), and on two planes transverse to the beamline and $700 \mathrm{~cm}$ from the interaction point (corresponds to the CSC station 1 in two muon endcaps). The muon trajectories are propagated to the cylinder when $|\eta|<1$ (barrel) and are propagated to one of the planes when $|\eta|>1$ (endcaps). The difference in azimuthal coordinate of the two muon trajectories on these surfaces are called $\Delta \phi$. Closeness in the longitudinal coordinate is quantified by $\Delta z / r$ on the cylinder and $\Delta r / z$ on the planes. Figures 5.2 and 5.3 show reconstruction efficiency as a function of $\Delta \phi$ with a constraint on $\Delta z / r$ and $\Delta r / z$ for the barrel and endcaps, respectively.

\subsubsection{Muon Track Requirements}

In this section we compare muon track related quality requirements with one of the standard recommended sets of requirements for CMS analyses with muons in the final state (Soft Muon selection) that requires:

- number of valid hits in the silicon tracker $>10$ 


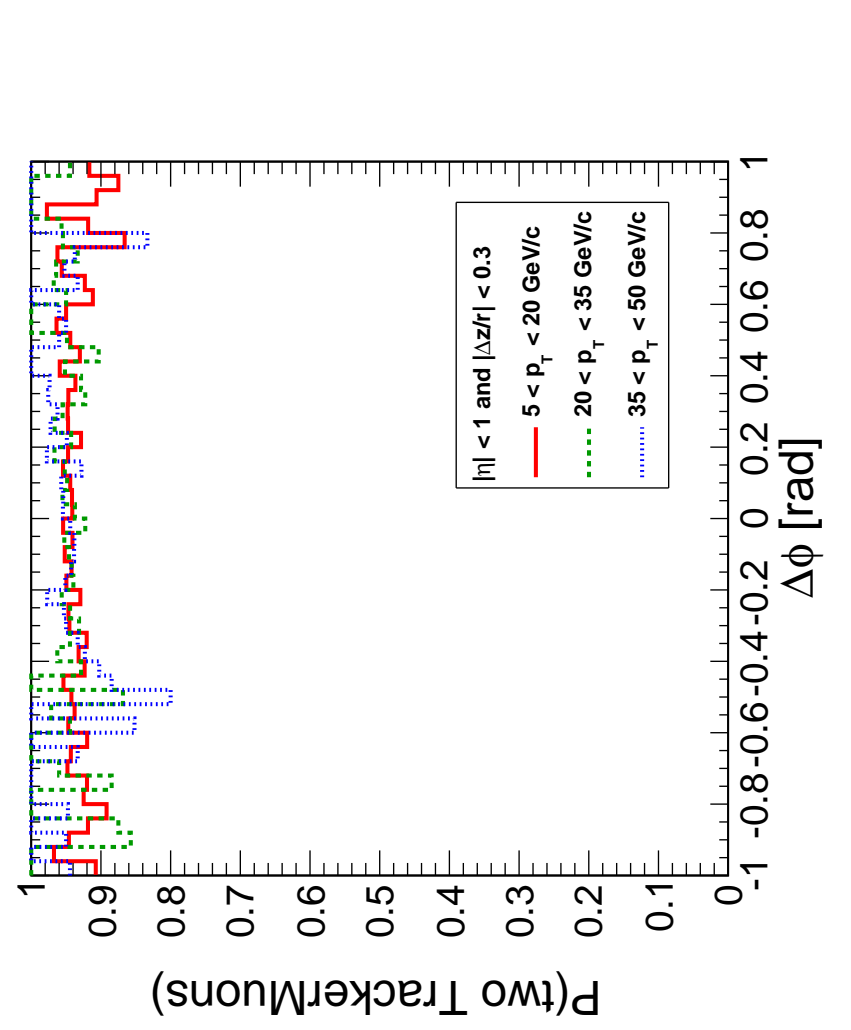

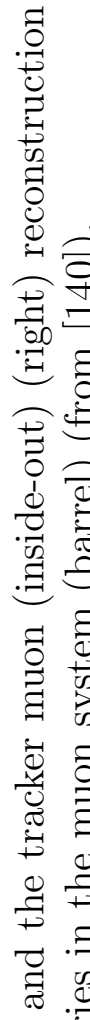

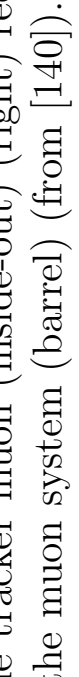

Ð્ટّ

$\nexists \frac{\pi}{\pi}$

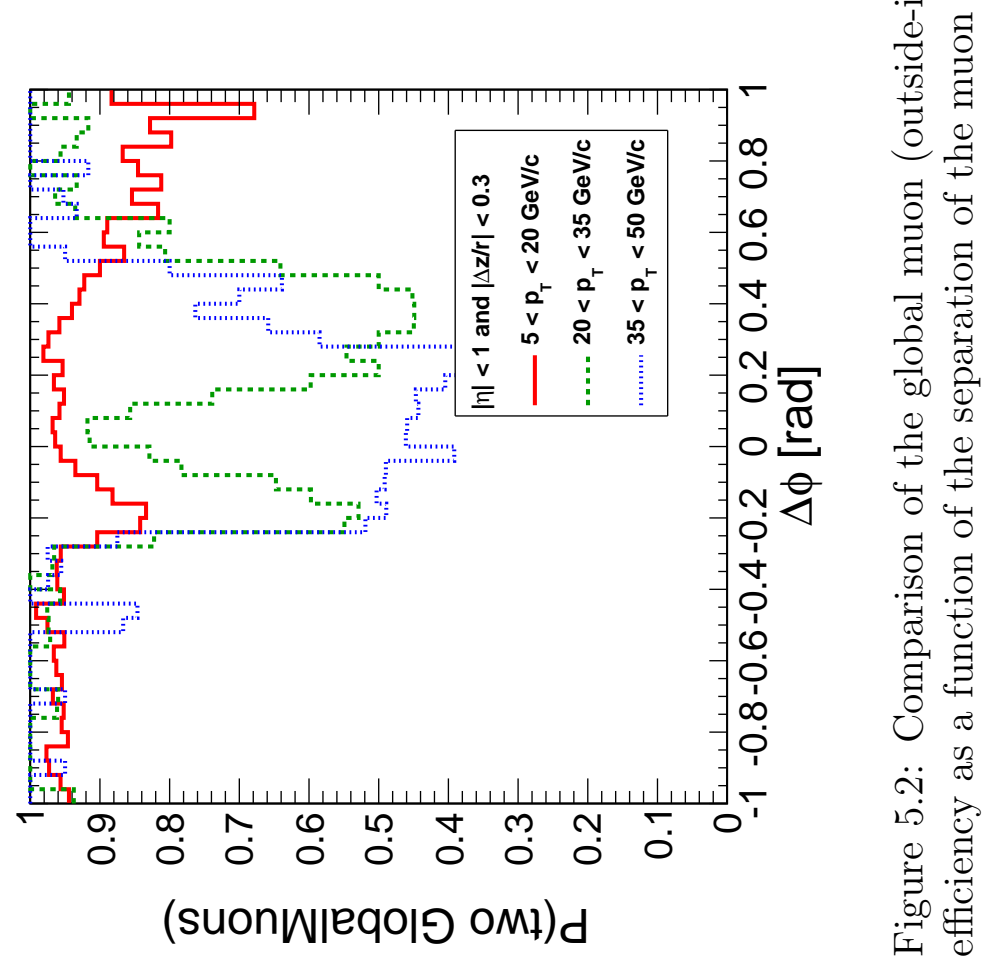



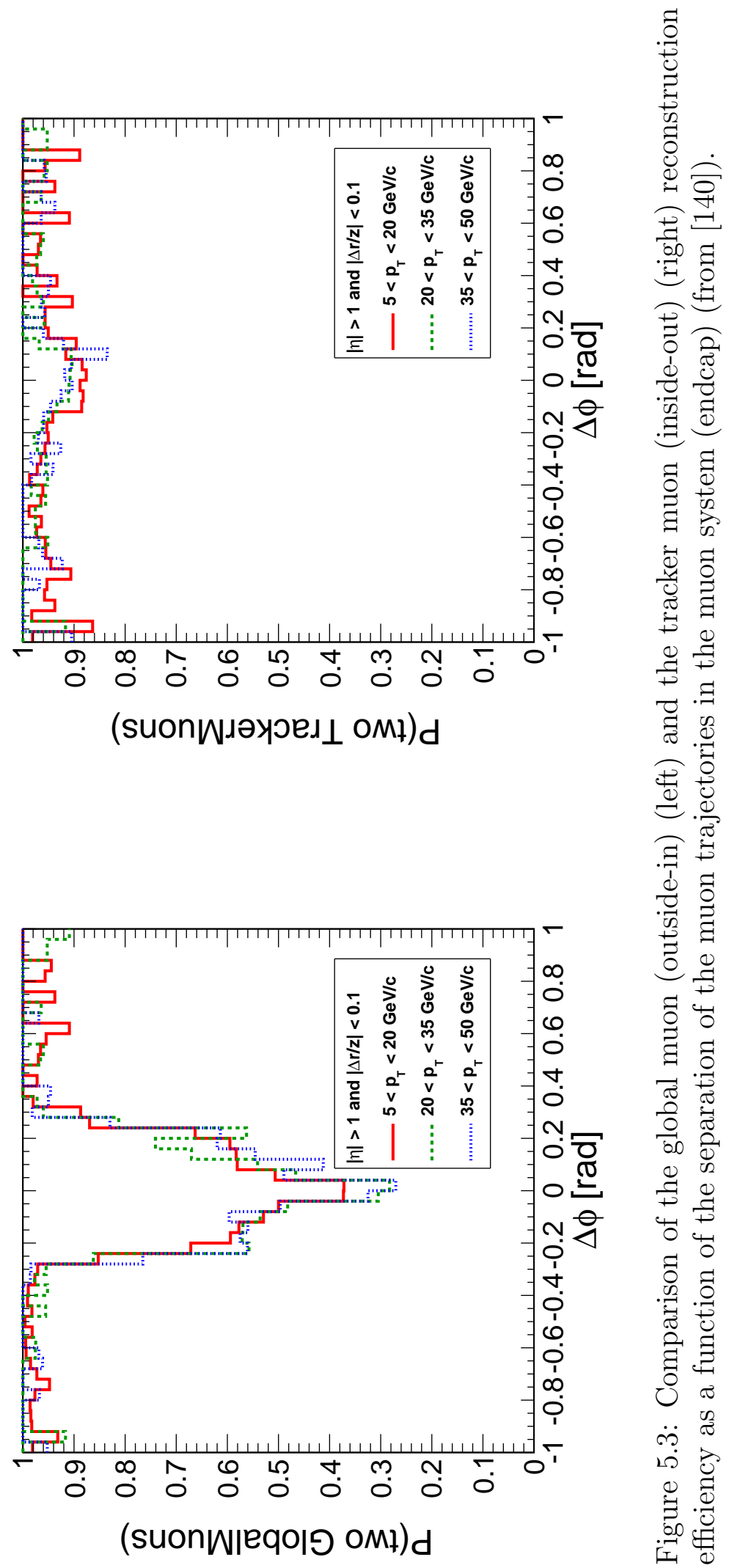
- normalized $\chi^{2}$ of track in the silicon tracker $<1.8$

- number of tightly matched muon segments $\geq 1$ (arbitrated)

- number of pixel layers $>1$

- $|d B|<3.0 \mathrm{~cm}$ and $|z|<30 \mathrm{~cm}$.

\begin{tabular}{|c|c|c|c|c|c|c|}
\hline \multicolumn{2}{c|}{$\begin{array}{c}\text { NMSSM } \\
\text { sample }\end{array}$} & \multicolumn{2}{c|}{$\begin{array}{c}\text { Analysis } \\
\text { selection }\end{array}$} & \multicolumn{2}{c|}{$\begin{array}{c}\text { Standard } \\
\text { Soft Muon selection }\end{array}$} \\
\hline$m_{h_{1}}, \mathrm{GeV}$ & $m_{a_{1}}, \mathrm{GeV}$ & $\alpha_{G E N}$ & $\alpha_{R E C O}$ & $\alpha_{R E C O} / \alpha_{G E N}$ & $\alpha_{R E C O}$ & $\alpha_{R E C O} / \alpha_{G E N}$ \\
\hline 100 & 0.25 & 47.0 & 34.9 & 74.2 & 19.3 & 41.1 \\
\hline 100 & 2 & 20.0 & 14.7 & 73.5 & 14.4 & 72.0 \\
\hline 125 & 2 & 27.5 & 20.0 & 72.6 & 18.8 & 68.4 \\
\hline
\end{tabular}

Table 5.1: Comparison of muon track related quality requirements in the analysis and the standard Soft Muon selection recommended by the CMS Muon POG shows full selection efficiency $\alpha_{R E C O}$ and efficiency of kinematic requirements on muon transverse momenta and pseudorapidities $\alpha_{G E N}$, as well as their ratio. All efficiencies are in per cents.

Table 5.1 summarizes both full selection efficiency $\alpha_{R E C O}$ and efficiency of kinematic requirements on muon transverse momenta and pseudorapidities $\alpha_{G E N}$, as well as their ratio for several benchmark samples (NMSSM) with varying masses of $m_{h_{1}}$ and $m_{a_{1}}$. It demonstrates that the standard selection is model dependent (the ratio of $\alpha_{R E C O}$ and $\alpha_{G E N}$ is not constant and depends on $\left.m_{a_{1}}\right)$, while this analysis selection provides higher $\alpha_{R E C O}$ and constant ratio of $\alpha_{R E C O}$ and $\alpha_{G E N}$.

The variation in efficiency for the case of standard selections is mainly due to the following three requirements: the minimum number of pixel layers, the normalized $\chi^{2}$, and the number of valid tracker hits. Individual effect of each requirement on 

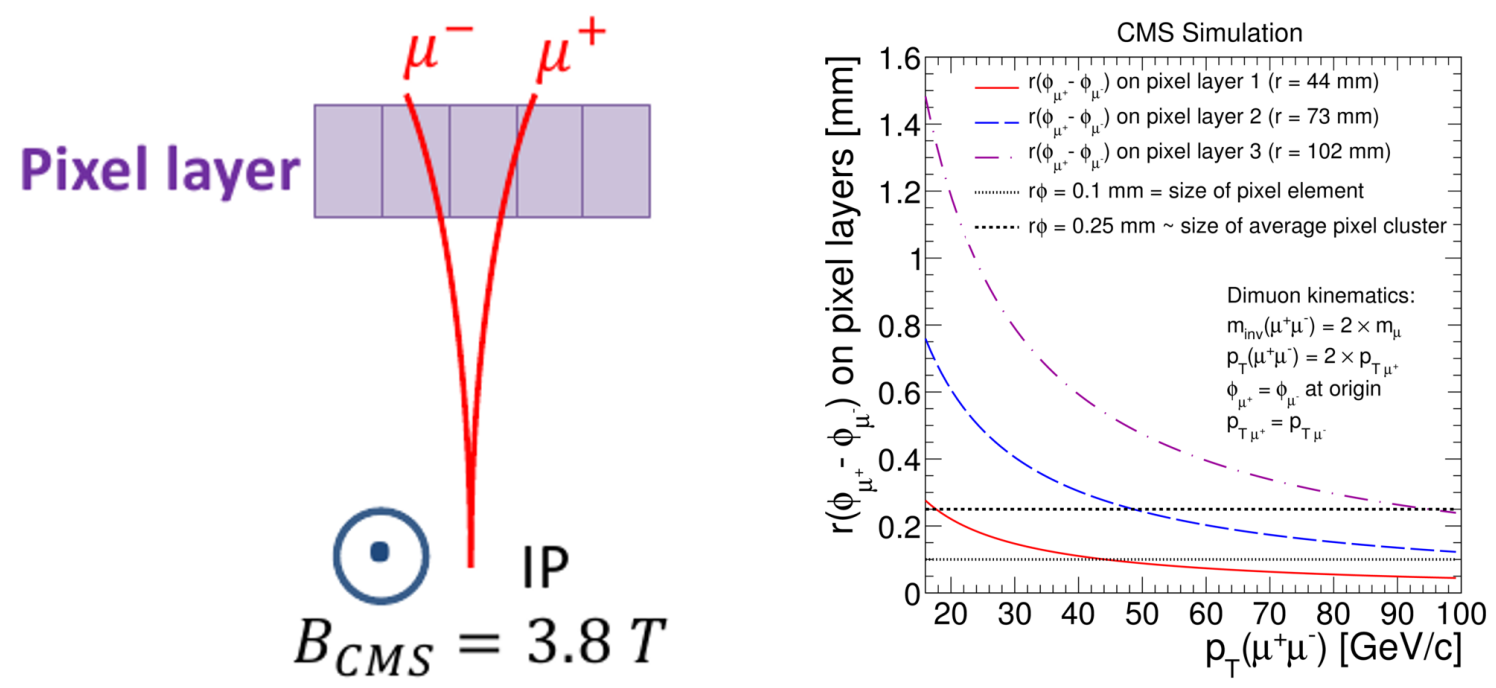

Figure 5.4: Left: sketch of the two close muon tracks of opposite sign crossing pixel layer in adjacent elements forming single shared cluster. Right: distance between two muon tracks in $r-\phi$ plane on three barrel layers of pixel tracker vs. transverse momentum of the dimuon $\left(p_{T}(d i-\mu)=p_{T}\left(a_{1}\right)\right)$ in the threshold case with $m(d i-\mu)=$ $2 \times m(\mu)$. Size of pixel element and typical cluster size are also shown as dashed horizontal lines.

top of the analysis requirements is shown in Table 5.2 for several NMSSM samples with varying $m_{a_{1}}$. The greatest offender is the requirement on the number of pixel layers, followed by the normalized $\chi^{2}$, and then by the requirement on the number of valid tracker hits. The mostly affected benchmark sample is the one with the lowest mass of $m_{a_{1}}=0.25 \mathrm{GeV}$.

The loss of selection efficiency when the mass of light boson is very low is due to the closeness of muon tracks in the pixel tracker. Typical distance between two muon tracks in $r-\phi$ plane on three barrel layers of pixel tracker with respect to transverse momentum of the light boson $a_{1}$ is shown in Fig. 5.4 (right). The two muons hit adjacent pixel elements almost always in the first layer, and frequently enough in the second layer, given typical momentum of the light boson. This is sketched in 


\begin{tabular}{|c|c|c|c|c|}
\hline \multirow{2}{*}{$\begin{array}{c}\text { NMSSM sample } \\
m_{h_{1}}=100 \mathrm{GeV} \\
m_{a_{1}}, \mathrm{GeV}\end{array}$} & $\begin{array}{c}\text { The analysis } \\
\text { selection (A.S.) }\end{array}$ & $\begin{array}{c}\text { A.S. \& number of } \\
\text { pixel layers } \geq 2\end{array}$ & $\begin{array}{c}\text { A.S. \& norm. } \\
\chi^{2}<1.8\end{array}$ & $\begin{array}{c}\text { A.S. \& valid } \\
\text { tracker hits }>10\end{array}$ \\
\hline 0.25 & $74.2 \pm 0.2$ & $52.1 \pm 0.7$ & $65.6 \pm 0.7$ & $70.9 \pm 0.7$ \\
\hline 0.5 & $74.3 \pm 0.9$ & $72.7 \pm 0.9$ & $71.1 \pm 0.9$ & $73.0 \pm 0.9$ \\
\hline 0.75 & $75.1 \pm 0.9$ & $73.7 \pm 1.0$ & $72.5 \pm 1.0$ & $74.0 \pm 1.0$ \\
\hline 1 & $73.6 \pm 1.0$ & $72.1 \pm 1.0$ & $71.8 \pm 1.0$ & $72.4 \pm 1.0$ \\
\hline 2 & $73.5 \pm 0.3$ & $72.7 \pm 1.0$ & $72.6 \pm 1.0$ & $73.5 \pm 1.0$ \\
\hline
\end{tabular}

Table 5.2: Individual effects of changes (from analysis selection to the standard selection) in three specific muon track related quality requirements on full selection efficiency $\alpha_{R E C O}$ and efficiency of kinematic requirements on muon transverse momenta and pseudorapidities $\alpha_{G E N}$, as well as their ratio. All efficiencies are in per cents.

Fig. 5.4 (left). Such close hits in pixel elements will be often combined into a single cluster.

To demonstrate the effect of such a combination, dimuons in NMSSM sample $\left(m_{h_{1}}=100 \mathrm{GeV}\right.$ and $\left.m_{a_{1}}=0.25 \mathrm{GeV}\right)$ are separated into different categories based on the number of reconstructed hits in the first layer of pixel tracker per dimuon with respect to parameter $d$, the distance in $r-\phi$ plane between the simulated hits of the same muons on the same layer of the pixel detector (see sketch on the left in Fig. 5.5). These categories are the following: (1) each muon in the pair has a distinct reconstructed hit, (2) muons share the same reconstructed hit, (3) the reconstructed hit assigned only to one muon in the pair and (4) no reconstructed hit assigned to any muon in the pair. Separation into the listed categories with respect to distance $d$ is shown in Fig. 5.5 (right). It is clearly seen, that if muon tracks are not close $(d>0.25 \mathrm{~mm}$, which is typical size of the cluster in a layer of pixel detector) then in most pairs each muon has a distinct reconstructed hit. When muon tracks are close $(d \leq 0.25 \mathrm{~mm})$, clusters can be assigned to one or none of the two muons which leads 
CMS Simulation
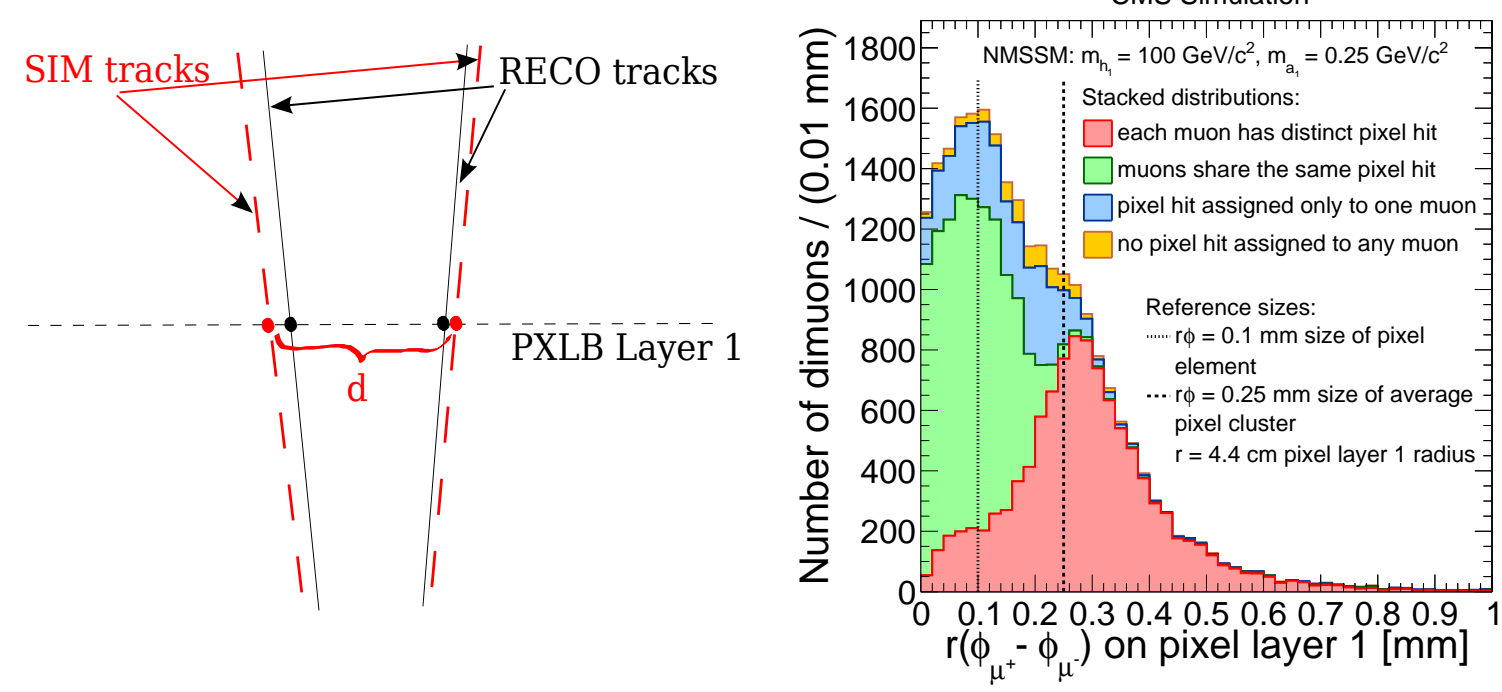

Figure 5.5: Left: sketch introducing parameter $d$ - distance in $r$ - $\phi$ plane between the simulated hits of the muons in the pair on the first layer of the pixel detector. Right: distribution (stacked) of number of dimuons in different categories of assigned reconstructed hits (see list in the text) vs. parameter $d$.

to performance degradation of the tracking reconstruction algorithm. No cluster assignment to any muon in the pair leads to a lower number of valid tracker hits and number of pixel layers. A shared cluster made from deposits of both muons in the pair is reconstructed to have a position somewhere between the true positions of the original hits associated with the two muon tracks. The displaced reconstruction of the hit increases the normalized $\chi^{2}$ of the track due to typically a very small uncertainty expected in the hit position measurements in the pixel tracker.

Effect of the shared single cluster on the $\chi^{2}$ distribution of the muon tracks is shown in Fig. 5.6 (left) for close dimuons $(d \leq 0.25 \mathrm{~mm})$ in the first layer of the pixel tracker for the NMSSM MC sample $\left(m_{h_{1}}=100 \mathrm{GeV}\right.$ and $\left.m_{a_{1}}=0.25 \mathrm{GeV}\right)$. It shows that tracks with reconstructed hit in this layer have the $\chi^{2}$ distribution shifted to larger values with respect to the distribution for tracks with lost hit on 

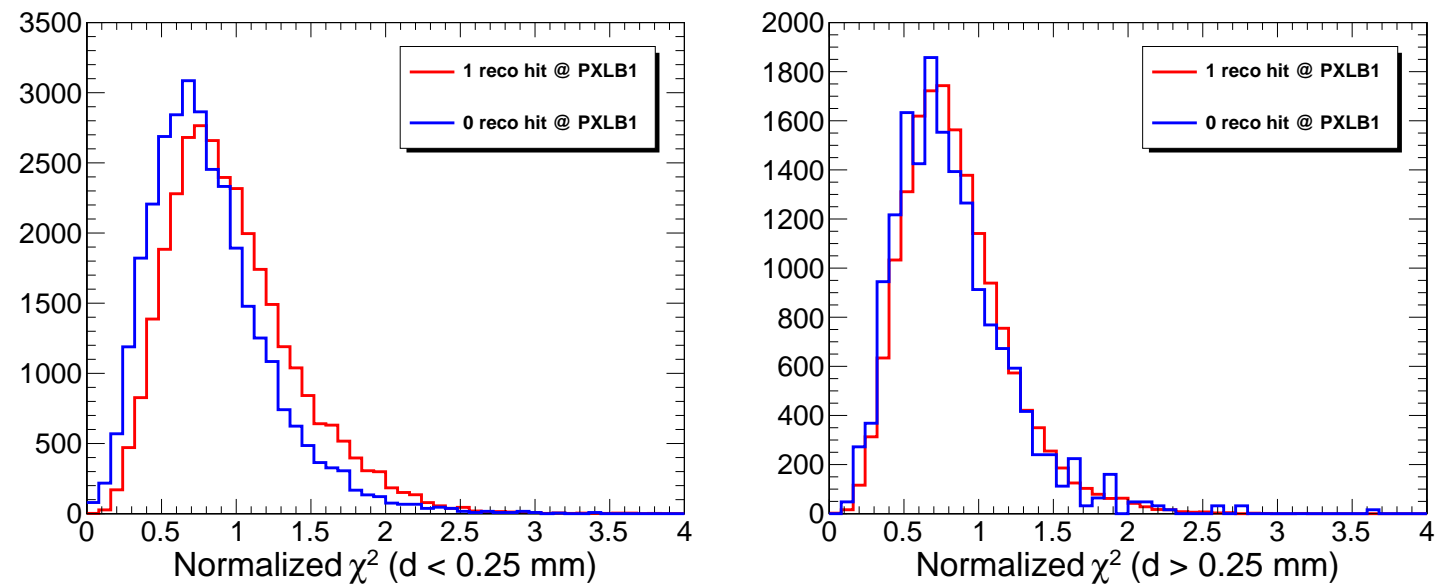

Figure 5.6: Distributions of the normalized $\chi^{2}$ for muon tracks that have only single shared reconstructed hit per dimuon or no assigned hit on the first layer of the pixel tracker. Distributions for muons which are close at the first layer of the pixel tracker are shown on the left, and for muons which are separated are shown on the right.

this layer. For comparison, $\chi^{2}$ distributions for muon tracks that are known to be far from each other $(d>0.25 \mathrm{~mm})$ at the first layer of the pixel tracker have the same shape whether the hit in the first layer of pixel tracker is lost or not, as shown in Fig. 5.6 (right). Thus, that standard requirement of normalized $\chi^{2}$ to be less than 1.8 leads to an efficiency loss for pairs of muons close in layers of pixel detector.

\subsubsection{Event Selection}

Events selected for the analysis are required to contain at least four muons passing offline muon candidate selection discussed earlier. In addition to the requirement for these muons to have transverse momenta $p_{T}>8 \mathrm{GeV}$, at least one of these muons is required to be in the barrel region $(|\eta|<0.9)$ and have transverse momentum $p_{T}>17$ $\mathrm{GeV}$ to ensure that the trigger efficiency for selected events is high and independent of pseudorapidity $\eta$ of either of the dimuons (see Sec. 5.2.2.1). In addition, events are required to have at least one primary vertex with at least 4 tracks and with the 
vertex global $z$ coordinate within $24 \mathrm{~cm}$ from the CMS origin.

All good quality muons in selected events are iteratively grouped into dimuons using the following algorithm. A pair of oppositely charged muons is grouped into a dimuon if they their invariant mass $m\left(\mu^{+} \mu^{-}\right)<5 \mathrm{GeV}$ and either they are compatible with coming from the the same vertex (the fit of the tracker tracks associated with the two muons for a common vertex has the vertex probability $P_{v}\left(\mu^{+} \mu^{-}\right)>1 \%$ ) or the two muons are within $\Delta R\left(\mu^{+} \mu^{-}\right)=\sqrt{\Delta \eta_{\mu^{+} \mu^{-}}^{2}+\Delta \phi_{\mu^{+} \mu^{-}}^{2}}<0.01$ of each other (see Sec. 5.2.2.2).

If any two dimuons share a muon, they are grouped into a "muon jet". Muon jets are grouped into a bigger muon jet, if they share one or more muons. The process of grouping stops when remaining muon jets don't share any muons. Selected events are allowed to contain muons that haven't been included in any dimuon ("orphan" muons). Rejection of events with orphan muons would introduce unnecessary model dependency (for example, if the Higgs boson production is associated with b-jets, semileptonic decays of the b-jets would lead to a rise of orphan muons).

Next, only events with exactly two dimuons are selected. This selection is over $96 \%$ efficient for the signal events, the inefficiency being due to the common vertex fit requirement.

For the remaining events, we reconstruct the position of dimuon "production vertices" at the beamline, $z_{\mu \mu_{1}}$ and $z_{\mu \mu_{2}}$ (see Sec. 5.2.2.3). Dimuons are required to originate from the same proton-proton collision, $\left|z_{\mu \mu_{1}}-z_{\mu \mu_{2}}\right|<1 \mathrm{~mm}$ (see Fig. 5.9(left)), and to be isolated from the rest of the event activity,

$$
I_{\text {trk }}^{a b s}(d i-\mu)=\sum_{\text {tracks }} p_{T}(\text { track })<3 \mathrm{GeV}
$$

where the summation runs over all reconstructed tracks with $p_{T}($ track $)>0.5 \mathrm{GeV}$ 
and satisfying $\Delta R($ track,$\mu \mu)=\sqrt{\Delta \eta^{2}(\operatorname{track}, \mu \mu)+\Delta \phi^{2}(\operatorname{track}, \mu \mu)}<0.4$ (dimuon direction is defined in Sec. 5.2.2.3), which are consistent with originating from the production vertex of the light boson as discussed above $\left(\left|z_{\text {track }}-z_{\mu \mu}\right|<1 \mathrm{~mm}\right)$, and excludes tracks associated with the muons forming the dimuon (see Fig. 5.13(left)). A typical efficiency of the dimuon isolation requirement is about $96 \%$ per dimuon in signal events, at the same time it reduces the $b \bar{b}$ background contribution by a factor of about 40. Adoption of the absolute dimuon isolation definition is not incidental: the relative dimuon isolation is $p_{T}$ dependent, thus, leading to undesired model dependence of the results (see Sec. 5.2.2.6).

The very final requirement is that the masses of the two dimuons reconstructed in the event should be consistent with each other within at least $5 \sigma$ of the core detector resolution (see Sec. 5.2.2.4):

$$
\left|m_{\mu \mu_{1}}-m_{\mu \mu_{2}}\right|<0.13+0.065 \times\left(m_{\mu \mu_{1}}+m_{\mu \mu_{2}}\right) / 2 .
$$

\begin{tabular}{|l|l|}
\hline HLT_DoubleMu* triggers & HLT_DoubleMu*_Mu* triggers \\
\hline HLT_DoubleMu6_v1 & HLT_DoubleMu13_Mu8_v2 \\
HLT_DoubleMu7_v1 & HLT_DoubleMu13_Mu8_v3 \\
HLT_DoubleMu7_v2 & HLT_DoubleMu13_Mu8_v4 \\
& HLT_DoubleMu13_Mu8_v6 \\
& HLT_DoubleMu13_Mu8_v7 \\
& HLT_DoubleMu17_Mu8_v2 \\
& HLT_DoubleMu17_Mu8_v3 \\
& HLT_DoubleMu17_Mu8_v4 \\
& HLT_DoubleMu17_Mu8_v6 \\
& HLT_DoubleMu17_Mu8_v7 \\
& HLT_DoubleMu17_Mu8_v10 \\
& HLT_DoubleMu17_Mu8_v11 \\
\hline
\end{tabular}

Table 5.3: List of the lowest threshold unprescaled inclusive non-isolated double muon triggers available during 2011 year. 


\subsubsection{Choice of Muon Trigger}

Inclusive non-isolated single muon triggers haven't been considered for this analysis as the muon transverse momentum thresholds for unprescaled triggers have been much higher than the thresholds in inclusive non-isolated double muon triggers available in year 2011 (thus, leading to a reduced efficiency of selecting signal events by the trigger). Table 5.3 lists all unprescaled inclusive non-isolated double muon triggers. We select events passing one of the DoubleMu17_Mu8 trigger versions. For runs in early 2011 when this trigger hasn't been available yet, therefore we emulate it by selecting events passing earlier versions of DoubleMu trigger and requiring presense of two muons, one with $p_{T}>17 \mathrm{GeV}$ and another one with $p_{T}>8 \mathrm{GeV}$ (event selection that follows all trigger changes during 2011 would introduce unnecessary complexity in future re-interpretation of analysis results).

Fig. 5.7(left) shows the trigger efficiency per event for simulated NMSSM signal events with $m_{h_{1}}=100 \mathrm{GeV}$ and $m_{a_{1}}=2 \mathrm{GeV}$. The efficiency is calculated for events with at least four muons with $p_{T}>8 \mathrm{GeV}$, of which one has to have $p_{T}>17 \mathrm{GeV}$, and is plotted as a function of $\eta$ of the leading muon. The diminished trigger efficiency in the endcap region introduces an undesired model dependence. To avoid it, in our analysis selections we require the presence of at least one muon with $p_{T}>17 \mathrm{GeV}$ and $|\eta|<0.9$. The trigger fiducial requirement leads to a loss in selection efficiency of signal events by about $20 \%$. However, once this requirement is applied, the trigger efficiency per event becomes high (96-97\%) and independent of $\eta$.

\subsubsection{Nearby Muons in Dimuons}

Fig. 5.8 shows distribution of spacial separation $\Delta R$ between muons in a dimuon for simulated NMSSM signal events with $m_{h_{1}}=100 \mathrm{GeV}$ and $m_{a_{1}}=0.25,0.5$, and $3 \mathrm{GeV}$. 


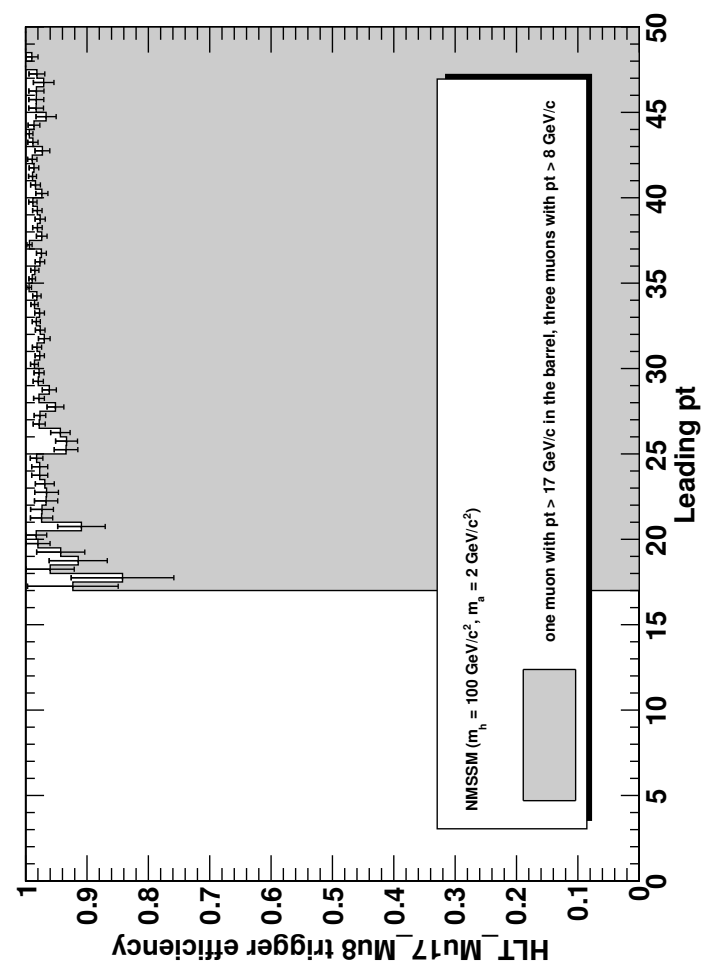

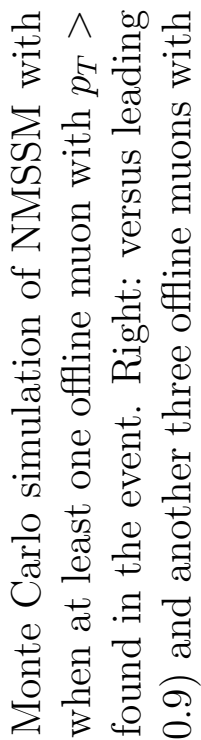

bo $\$: \cup$

क 60

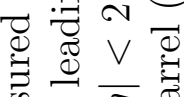

可

过高

की

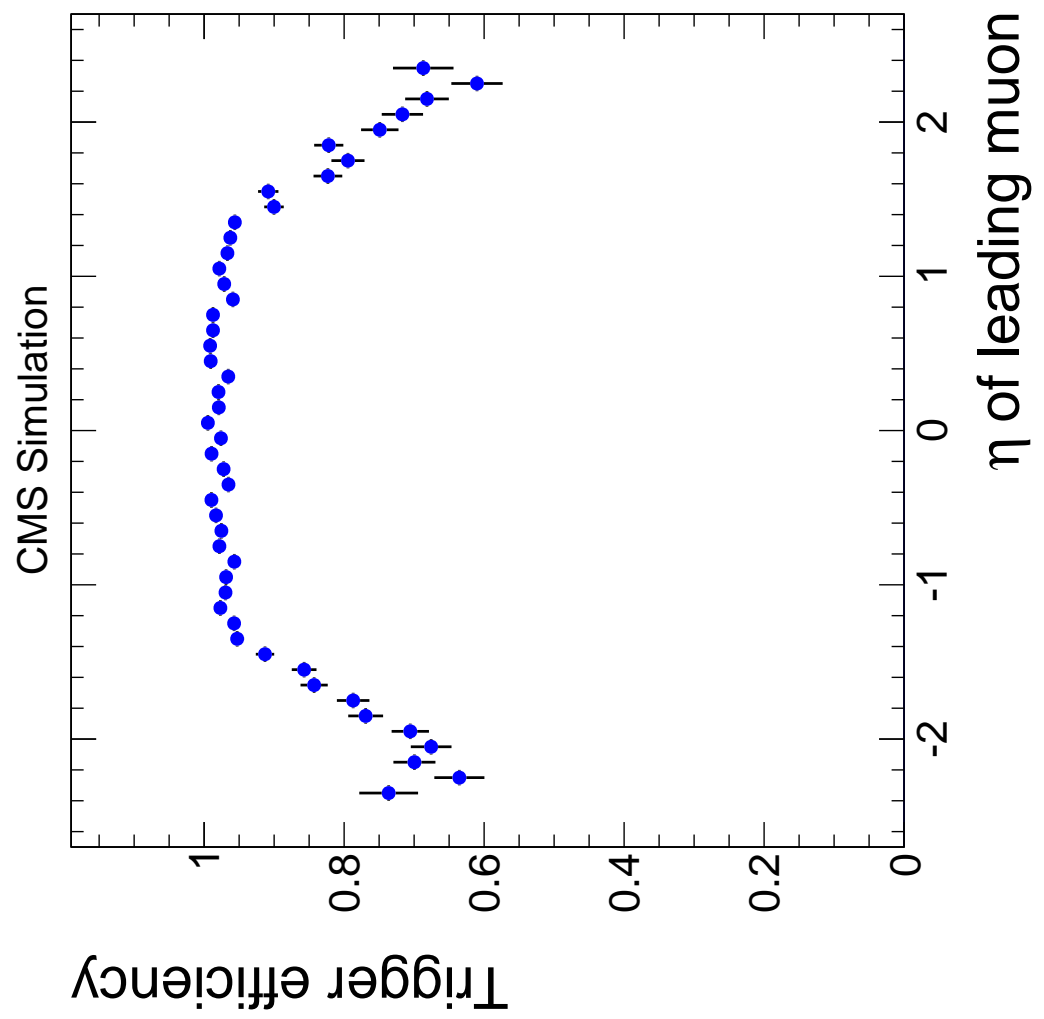

至>> • $\rightleftarrows \nsubseteq$ $\rightarrow \circ \wedge$ $\infty_{0}^{\infty} \approx+15$

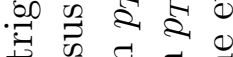

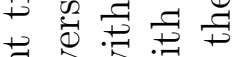
व $>$ 今

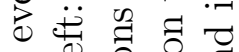

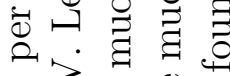

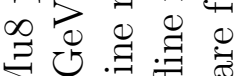

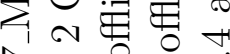
기이

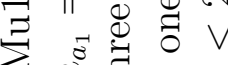
$\lambda_{1}$ क

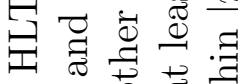

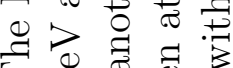

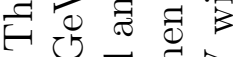
듀 20 $>5$

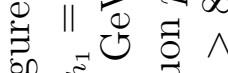

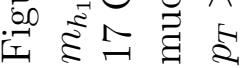


When constructing dimuons, we require muons to be consistent with originating from the same new light boson by introducing the common vertex fit requirement. However, when the muons in a dimuon are very close $(\Delta R<0.01)$, the efficiency of the common vertex fit requirement is significantly reduced. In these cases, the requirement can be safely dropped as this doesn't introduce any significant increase in the background contribution as the probability of two unrelated muon candidates being so close to each other is negligible.
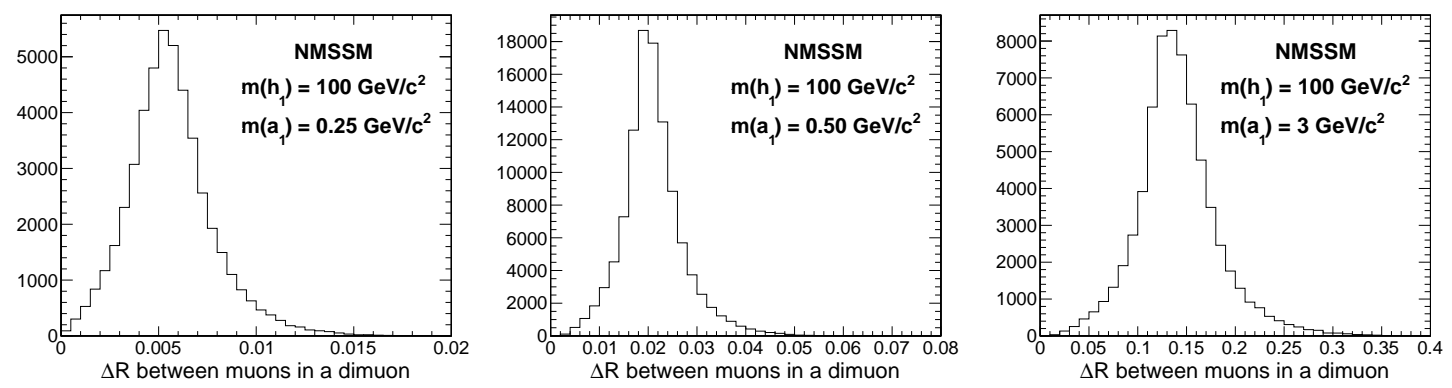

Figure 5.8: Distribution of spacial separation $\Delta R$ between muons in a dimuon in NMSSM Monte Carlo samples with $m_{h_{1}}=100 \mathrm{GeV}$ and $m_{a_{1}}=0.25,0.5$, and 3 $\mathrm{GeV}$.

\subsubsection{Dimuon Production Vertex}

Reconstruction of the position of dimuon "production vertex" at the beamline $z_{\mu \mu}$ starts with the calculation of the $3 \mathrm{D}$ direction of the dimuon momentum $\overrightarrow{p_{\mu^{+}}}+\overrightarrow{p_{\mu^{-}}}$at the position of the reconstructed "common vertex" of the two muons (for muons with very close directions where the vertex is not reconstructed, momenta are taken at the points on the respective trajectories where the two tracks are the closest to each other in $x y$-plane). Next, a straight line is projected along the dimuon direction vector from the common vertex (or the point in the middle of the segment connecting the 

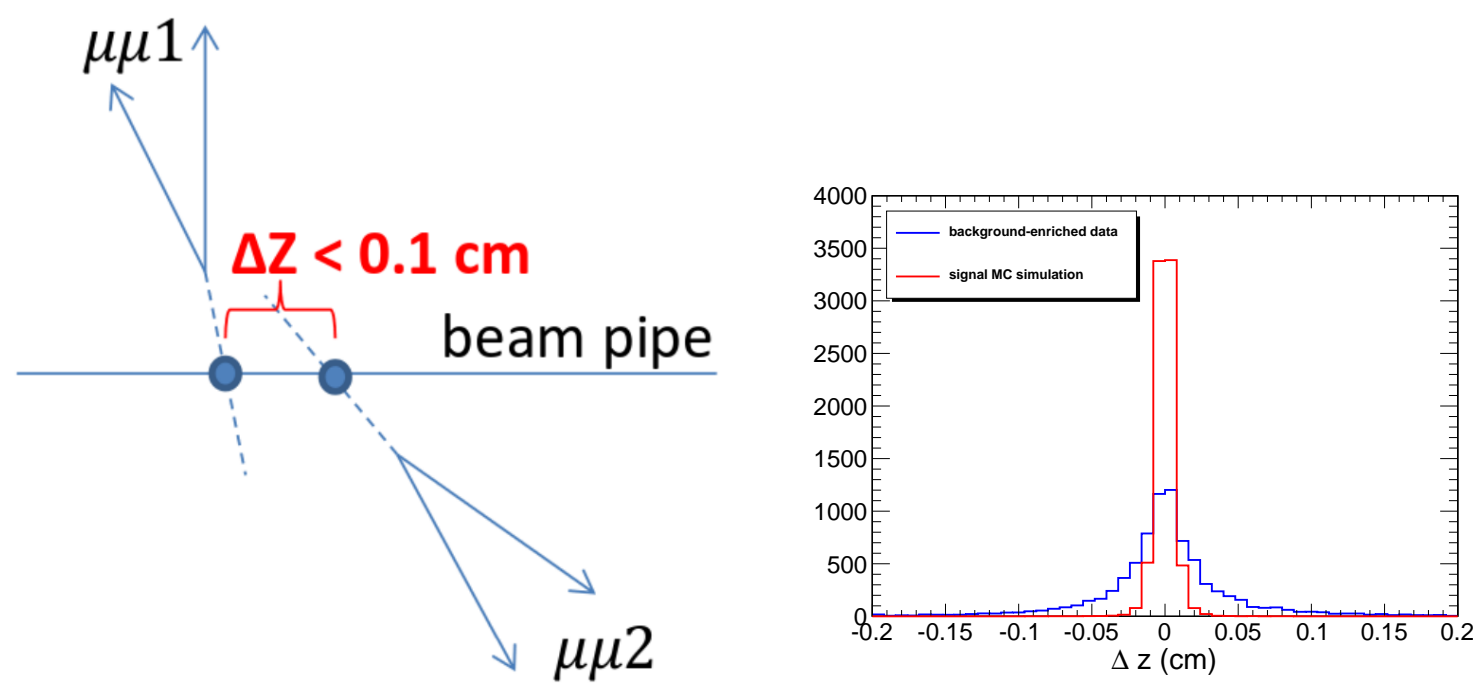

Figure 5.9: Left: sketch demonstrating reconstruction of the position of dimuon "production vertex" at the beamline, $z_{\mu \mu}$. Two dimuons are required to be originating from the same proton-proton collision: $\left|z_{\mu \mu_{1}}-z_{\mu \mu_{2}}\right|<1 \mathrm{~mm}$. Right: $\left|z_{\mu \mu_{1}}-z_{\mu \mu_{2}}\right|$ measured for two dimuons in the NMSSM Monte Carlo sample with $m_{h_{1}}=100 \mathrm{GeV}$ and $m_{\left(a_{1}\right.}=2 \mathrm{GeV}$ and for dimuon and orphan muon in events from backgroundenriched part of data.

points on the trajectories where momenta of the two muons are calculated) towards the beamline. The $z$-coordinate of the projected line at the point of closest approach to the beamline in the $x y$-plane becomes the reconstructed $z_{\mu \mu}$ of the "production vertex" (see Fig. 5.9(left)).

The requirement of dimuons to be originating from the same proton-proton collision $\left|z_{\mu \mu_{1}}-z_{\mu \mu_{2}}\right|<1 \mathrm{~mm}$ is safe as the typical distance between the $z$ positions of two nearby collisions is substantially larger than $1 \mathrm{~mm}$ (if the size of the highly luminous region is taken to be $\sim 15 \mathrm{~cm}$, for an event with $\sim 30$ pile-up collisions the "average distance" between the interactions would be of the order of $0.5 \mathrm{~cm}$ ). At the same time, the requirement is loose enough to ensure that signal events are never rejected by this selection, which is illustrated in Fig. 5.9(right). The red distribution shows 
the distribution of $z_{\mu \mu_{1}}-z_{\mu \mu_{2}}$ for the NMSSM simulated events with $m_{h_{1}}=100 \mathrm{GeV}$ and $m_{a_{1}}=2 \mathrm{GeV}$. The blue distribution shows $z_{\mu \mu}-z_{\mu}$ using a $b \bar{b}$ dominated data sample with events selected by requiring presence of exactly one dimuon and one orphan muon.

\subsubsection{Dimuon Mass Resolution}

At the final stage of the analysis, two selected dimuons are required to be consistent with each originating from the same type of particle. We require them to have invariant masses consistent within $5 \sigma$ of the core dimuon mass resolution. Since the resolution is fully determined by the detector resolution effects, it has been studied [140] using four narrow, high cross-section dimuon resonances in our mass range of interest: $\omega, \phi, J / \psi$, and $\psi^{\prime}$. Fig. 5.10 and 5.11 show mass distributions of these resonances separately in the barrel and endcap regions fitted with the Crystal Ball shape:

$$
\begin{aligned}
& C B\left(m ; m_{0}, \sigma, \alpha, n\right)= \\
& \frac{1}{\sqrt{2 \pi} \sigma}\left\{\begin{array}{cl}
\exp \left(-\frac{\left(m-m_{0}\right)^{2}}{2 \sigma^{2}}\right) & \text { if }\left(m-m_{0}\right) / \sigma>-\alpha \\
\exp \left(-\frac{\alpha^{2}}{2}\right)\left[\frac{n}{n-\alpha^{2}-|\alpha|\left(m-m_{0}\right) / \sigma}\right]^{n} & \text { otherwise }
\end{array}\right.
\end{aligned}
$$

Mass resolution for resonances selected from data as well as for simulated dimuons as a function of dimuon mass for several ranges of dimuon transverse momenta $p_{T}$ (from 20 to $100 \mathrm{GeV}$ ) separately in the barrel and endcap regions is shown in Fig. 5.12. The middle of the band in the endcap region that evolves with the mass of the resonance has been used for conservative estimate of the dimuon mass resolution: $\sigma(m)=0.026+0.013 m \mathrm{GeV}$ (for simplicity, the dimuon $p_{T}$ dependency is not 

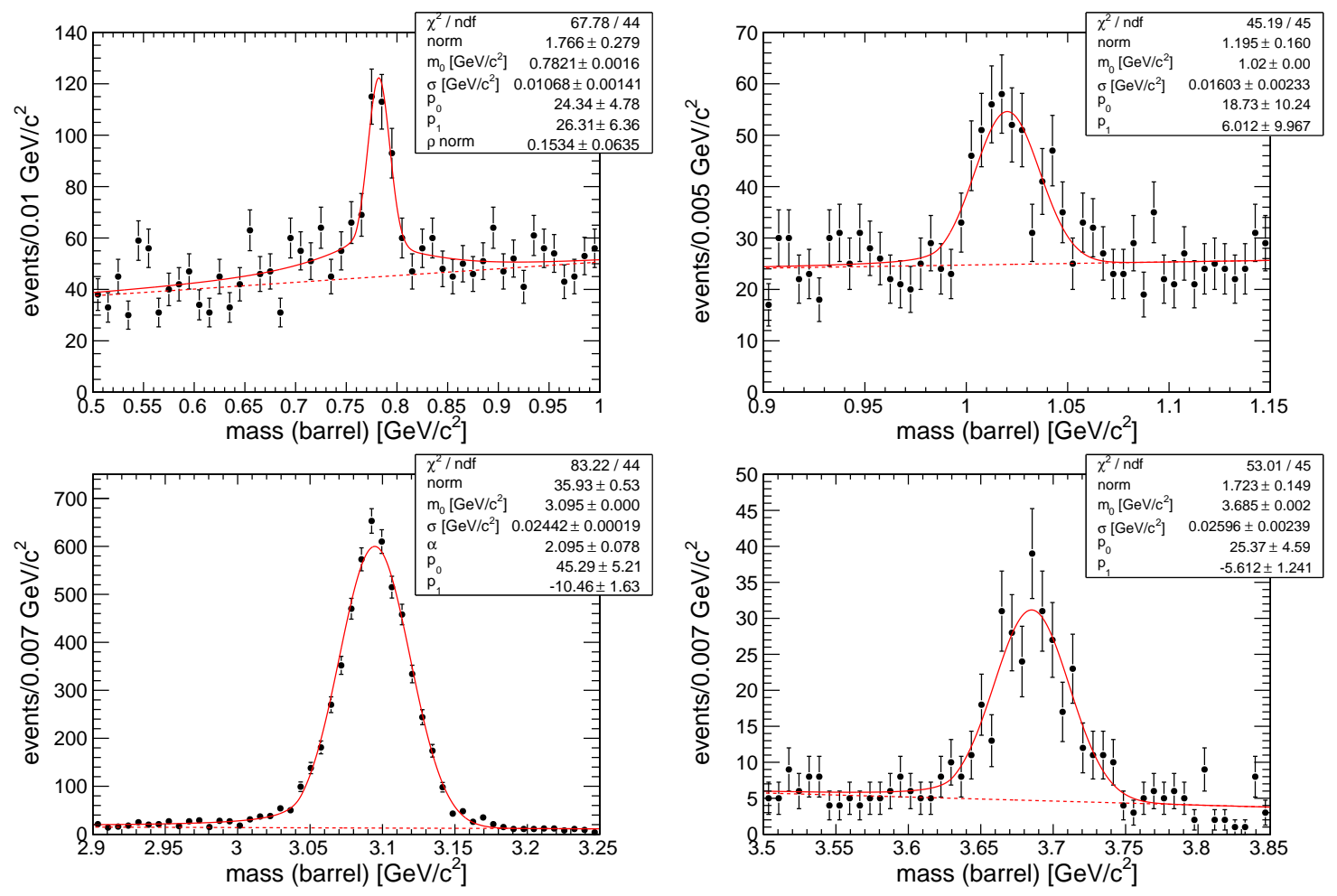

Figure 5.10: Four resonance mass peak fits in dimuon data: $\omega, \phi, J / \psi$, and $\psi^{\prime}$ in the barrel region $|\eta|<0.9$. Signal shapes are fitted to Crystal Ball function with $\alpha$ parameter floating only in the $J / \psi$ barrel case. Backgrounds are linear, and the $\omega$ fit has an additional $\rho$ background component with $\rho$ mass and width taken from the PDG.

included as the dimuon mass resolution weakly depends on dimuon $p_{T}$ ). The signal region in the two-dimensional distribution of dimuon invariant masses is defined as a "corridor" near the diagonal with the width of $5 \sigma$ in core detector resolution in each direction.

\subsubsection{Signal Region Width}

The width of the signal region in terms of dimuon mass resolution should be broad enough to not lose too much efficiency as dimuon mass distribution has a radiative tail that is not accounted by the resolution measured from the core part of the shape. 

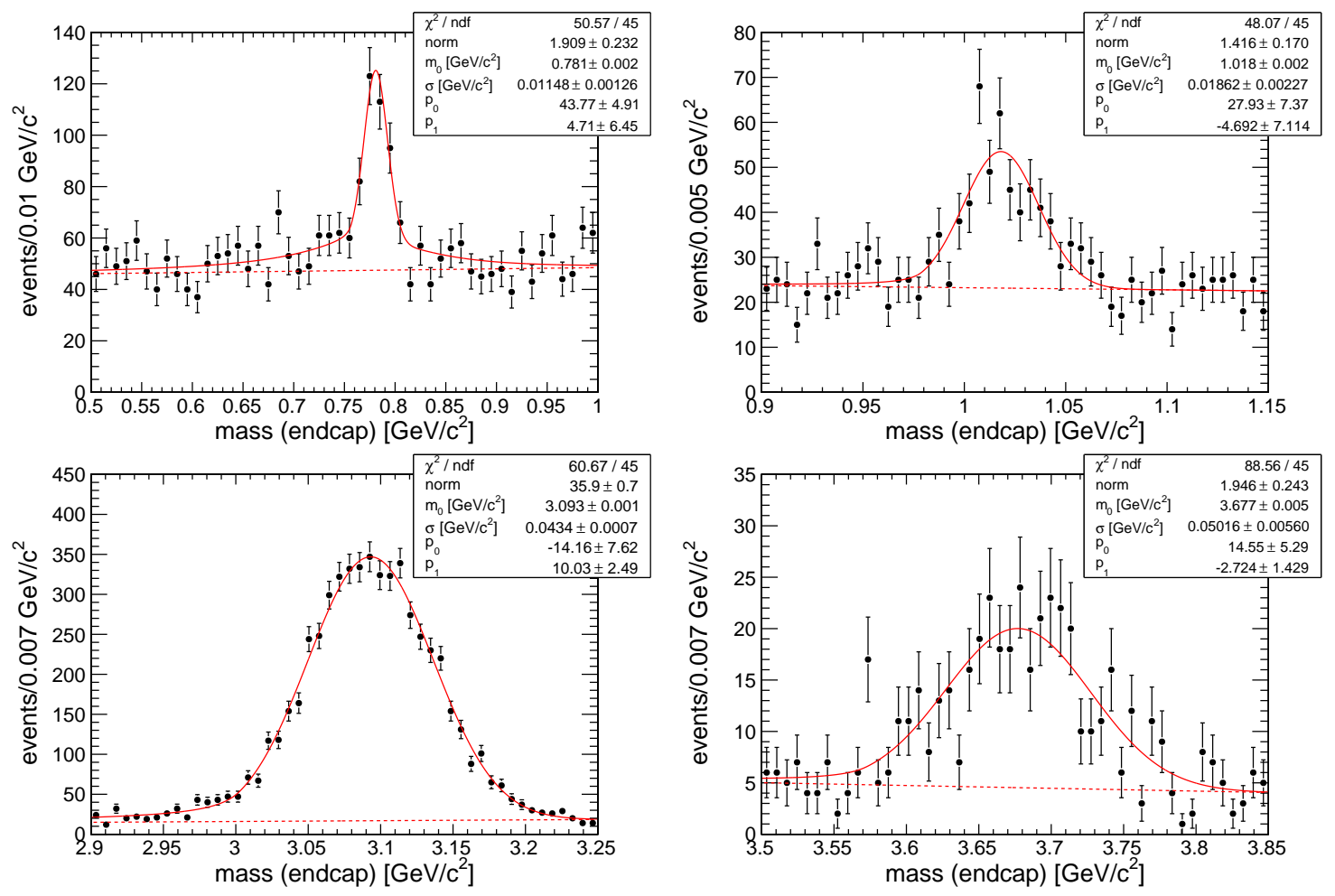

Figure 5.11: Four resonance mass peak fits in dimuon data: $\omega, \phi, J / \psi$, and $\psi^{\prime}$ in the endcap region $|\eta|>0.9$. Signal shapes are fitted to Crystal Ball function with $\alpha$ parameter floating only in the $J / \psi$ barrel case. Backgrounds are linear, and the $\omega$ fit has an additional $\rho$ background component with $\rho$ mass and width taken from the PDG.

Here we provide a study of several choices of the size of the signal region corridor in terms of $n \cdot \sigma$, where $n=1,3,5,7$ and $\sigma$ is the dimuon mass resolution. Signal acceptance efficiencies for several NMSSM MC benchmark samples and different values of the corridor width are shown in Table 5.4. Note that default value for signal corridor width used in the analysis is $n=5$ and corresponding acceptance efficiencies are within range 96.5-99.9\% depending on the signal sample. For further estimates we use the signal sample with $m_{a}=3 \mathrm{GeV}$ as the one with the lowest signal efficiency compared to other mass points.

For unbiased optimization of the signal region width for the best limit setting 

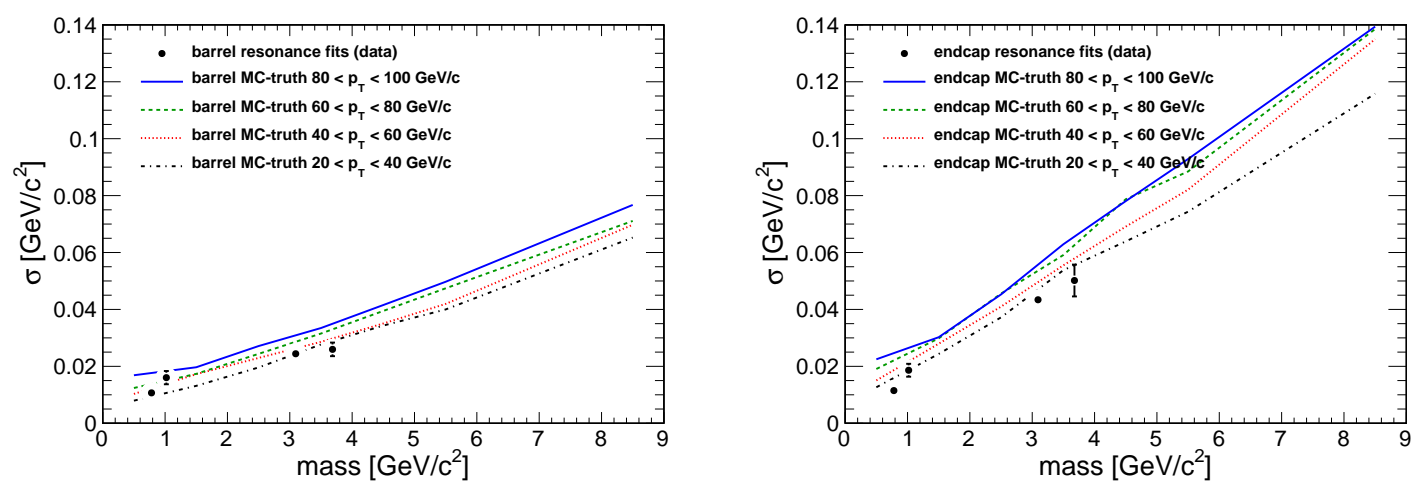

Figure 5.12: Left: reconstructed invariant mass resolution as a function of the true mass for simulated dimuons (lines) and real resonances (points) in the barrel region $|\eta|<0.9$ as obtained using the fit to a Crystal Ball shape. Right: the same distributions for the endcap region $|\eta|>0.9$.

potential, we follow the model rejection potential technique described in [141, 142]. The method introduces the concept of "average upper limit", calculated from a series of Poisson counting experiments with no true signal. The optimal choice of the signal region width corresponds to the minimal average upper limit among limits calculated for all considered choices.

We estimate the average weighted expected 95\% C.L. upper limit on the rate of signal events before applying the requirement that the events should fall into the signal region. For this calculation, we assume counting experiments for the entire signal region:

$$
R_{95 \% C L}=\frac{1}{\epsilon_{S}} \cdot\left[3 \cdot P\left(N=0, \nu_{B}\right)+4.74 \cdot P\left(N=1, \nu_{B}\right)+6.3 \times P\left(N \geq 2, \nu_{B}\right)\right]
$$

where the numerical factors are the bayesian upper $95 \%$ C.L. exclusions in terms of the rate of signal events for experiments with observed 0, 1 and 2 events, which are weighted by the Poisson probabilities to observe 0 , 1, or 2 and more events given 


\begin{tabular}{|c|c|c|c|c|}
\hline$m_{a_{1}}[\mathrm{GeV}]$ & \multicolumn{3}{|c|}{ Efficiency of $\left|m_{1}-m_{2}\right|<n \cdot \sigma$ requirement [\%] } \\
\cline { 2 - 5 } & $n=1$ & $n=3$ & $n=5$ & $n=7$ \\
\hline 0.25 & $95.8 \pm 0.1$ & $99.6 \pm 0.1$ & $99.9 \pm 0.1$ & $99.8 \pm 0.1$ \\
\hline 0.5 & $93.9 \pm 0.2$ & $99.0 \pm 0.1$ & $99.7 \pm 0.1$ & $99.8 \pm 0.1$ \\
\hline 0.75 & $91.6 \pm 0.2$ & $98.2 \pm 0.1$ & $99.2 \pm 0.1$ & $99.4 \pm 0.1$ \\
\hline 1 & $90.3 \pm 0.1$ & $97.8 \pm 0.1$ & $99.0 \pm 0.1$ & $99.4 \pm 0.1$ \\
\hline 2 & $83.5 \pm 0.1$ & $95.8 \pm 0.1$ & $97.5 \pm 0.1$ & $98.2 \pm 0.1$ \\
\hline 3 & $78.1 \pm 0.1$ & $94.5 \pm 0.1$ & $96.5 \pm 0.1$ & $97.6 \pm 0.1$ \\
\hline
\end{tabular}

Table 5.4: Acceptance efficiencies per event for several NMSSM Monte Carlo samples with $m_{h_{1}}=100 \mathrm{GeV}$ and $m_{a_{1}}$ varying from 0.25 to $3 \mathrm{GeV}$ as a function of the signal corridor width requirement.

expected background rate $\nu_{B}$. This expression is a slight simplification of the true limit calculation, but it is sufficient for the purposes of this study.

The background rate $\nu_{B}$ expected in the signal region is estimated in two steps:

- First, we relate the rate of background events in the signal and off-diagonal regions. To calculate the ratio of the two, $\epsilon_{B}$, we use $2 \mathrm{D}$ background shape template that will be obtained in Sec. 5.4. $\epsilon_{B}$ is defined as the ratio of the integrals of the background shape in the signal and the off-diagonal regions.

- Next, we need to determine the background rate in the off-diagonal region. Here we jump ahead and use results that will be obtained in Sec. 5.6. Just before opening the signal region, we have looked into the off-diagonal region and observed 3 events there. Since this region is dominated by background events, we constrain the normalization of the background rate in the off-diagonal region using the measured number of observed events.

- Then, the estimate of the background rate in the signal region is provided by scaling the background rate in the off-diagonal region by $\epsilon_{B}: \nu_{B}=3 \cdot \epsilon_{B}$. 
The calculation is repeated for each signal region size choice $(n=1,3,5,7)$. Table 5.5 shows that the upper $95 \%$ C.L. limit for the default choice of the signal region (corresponding to $5 \sigma$ ) is close to the optimal value.

\begin{tabular}{|c|c|c|c|c|}
\hline & $n=1$ & $n=3$ & $n=5$ & $n=7$ \\
\hline \hline$\epsilon_{S}\left(m_{h_{1}}=100 \mathrm{GeV} m_{a_{1}}=3 \mathrm{GeV}\right)$ & 0.78 & 0.94 & 0.97 & 0.98 \\
\hline$\epsilon_{B}$ & 0.073 & 0.13 & 0.18 & 0.23 \\
\hline \hline$\nu_{B}=3 \cdot \epsilon_{B}$ & 0.24 & 0.46 & 0.67 & 0.91 \\
\hline$P_{0}=\operatorname{Poisson}\left(N=0, \nu_{B}\right)$ & 0.79 & 0.63 & 0.51 & 0.40 \\
\hline$P_{1}=\operatorname{Poisson}\left(N=1, \nu_{B}\right)$ & 0.19 & 0.29 & 0.34 & 0.37 \\
\hline$P_{2}=\operatorname{Poisson}\left(N \geq 2, \nu_{B}\right)$ & 0.024 & 0.077 & 0.15 & 0.23 \\
\hline$\left\langle R_{95 \% \text { C.L. }}\right\rangle$ & 4.4 & 4.0 & 4.2 & 4.5 \\
\hline
\end{tabular}

Table 5.5: Efficiencies of the signal corridor requirement for the signal events $\left(\epsilon_{S}\right)$ in NMSSM sample with $m_{h_{1}}=100 \mathrm{GeV}$ and $m_{a_{1}}=3 \mathrm{GeV}$ and for the background events $\left(\epsilon_{B}\right),\left\langle R_{95 \%}\right.$ C.L. $\rangle$ is the estimate of the average expected $95 \%$ C.L. upper limit on the rate of signal events before applying the signal region requirement. The calculation is repeated for several choices of the corridor size parametrized with $n \cdot \sigma$. Background rate estimate is based on the 3 events observed in the off-diagonal region in the actual analysis.

\subsubsection{Dimuon Isolation}

Fig. 5.13 (right) shows the distribution of dimuon isolation of dimuons in NMSSM Monte Carlo sample with $m_{h_{1}}=100 \mathrm{GeV}$ and $m_{\left(_{1}\right.}=2 \mathrm{GeV}$ and for dimuons in the background-enriched sample (one dimuon plus exactly one orphan muon). The choice of the optimal value for dimuon isolation requirement utilizes a procedure similar to the one used for the optimization of the signal region width.

This time we use the NMSSM Monte Carlo sample with $m_{h_{1}}=100 \mathrm{GeV}$ and $m_{\left(a_{1}\right.}=2 \mathrm{GeV}$ and consider several choices for dimuon isolation requirement (1, $2,3,4,5 \mathrm{GeV})$. To evaluate the rate of background events in the signal region, 

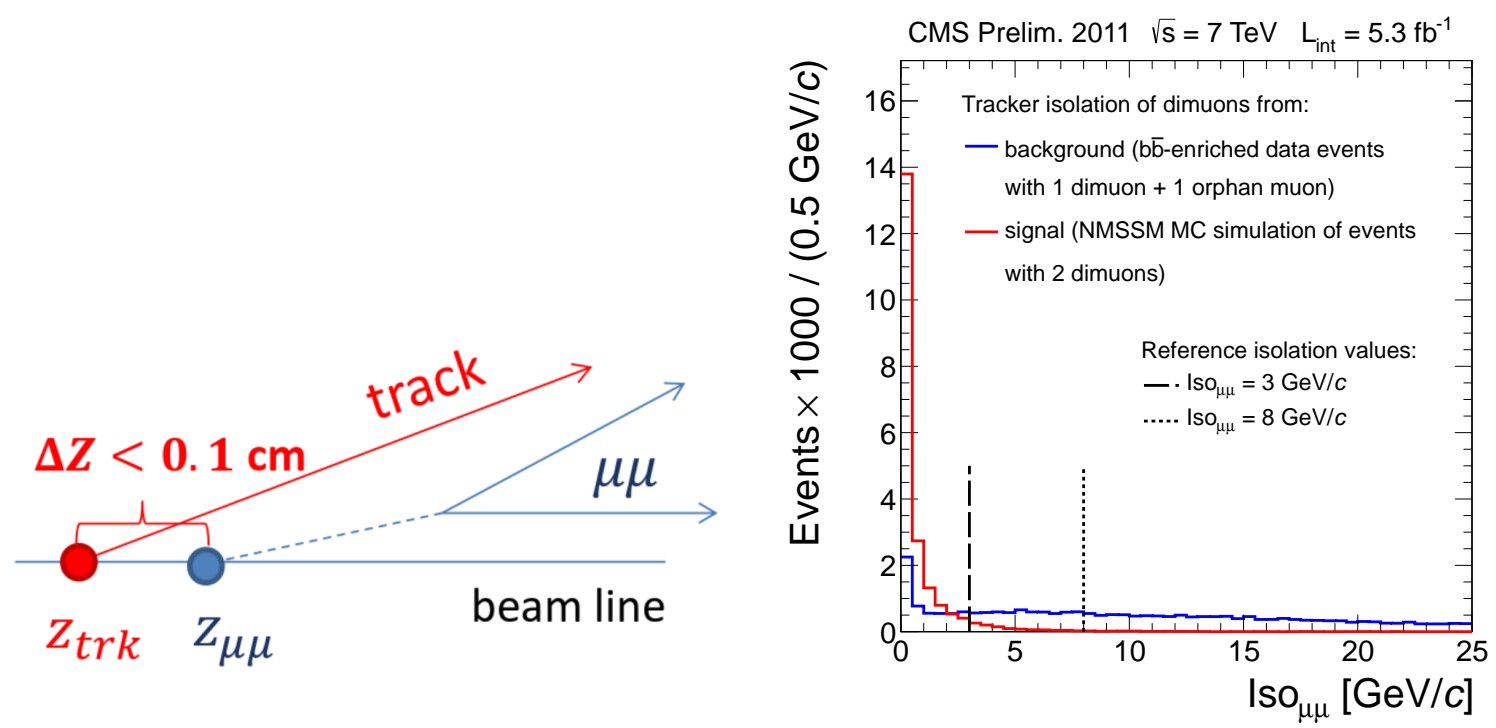

Figure 5.13: Left: sketch illustrating tracks used into the dimuon isolation. Right: dimuon isolation distribution for the reconstructed dimuons in NMSSM Monte Carlo sample with $m_{h_{1}}=100 \mathrm{GeV}$ and $m_{a_{1}}=2 \mathrm{GeV}$ and in events from backgroundenriched part of data.

we again constrain the background rate normalization using three events observed in the off-diagonal region after applying all selections except the requirement that events fall into the diagonal signal region, and rescale it to obtain the expected rate of background events in the signal region, which is 0.88 events, according to the background shape template. We then calculate the fraction of background events $\left(\epsilon_{B}^{\mu \mu}\right)$ passing each of the isolation cut values chosen (these fractions are calculated per dimuon using the background enriched data sample with one dimuon and one orphan muon). The efficiency per event $\left(\epsilon_{B}^{e v t}\right)$ is equal to the square of the efficiency per dimuon. The fractions are summarized in Table 5.6. Next, we calculate the average weighted $95 \%$ C.L. upper limit on the rate of signal events $\left\langle R_{95 \%}\right.$ C.L. $\rangle$ before the isolation selections is applied for a counting experiment. The default choice $I s o_{\mu \mu}<3 \mathrm{GeV}$ is reasonably close to the most optimal value, while maintaining high 
signal efficiency.

\begin{tabular}{|c|c|c|c|c|c|}
\hline & \multicolumn{5}{|c|}{ Iso $o_{\mu \mu}[\mathrm{GeV}]$} \\
\cline { 2 - 6 } & 1 & 2 & 3 & 4 & 5 \\
\hline \hline$\epsilon_{S}\left(m_{h_{1}}=100 \mathrm{GeV}, m_{a_{1}}=2 \mathrm{GeV}\right)$ & 0.81 & 0.87 & 0.92 & 0.94 & 0.96 \\
\hline$\epsilon_{B}^{\mu \mu}$ & 0.091 & 0.13 & 0.16 & 0.19 & 0.23 \\
\hline$\epsilon_{B}^{\text {evt }}=\left(\epsilon_{B}^{\mu \mu}\right)^{2}$ & 0.0083 & 0.016 & 0.026 & 0.038 & 0.054 \\
\hline \hline$\nu_{B}$ & 0.21 & 0.41 & 0.67 & 0.98 & 1.4 \\
\hline$\left\langle R_{95 \% \text { C.L. }}\right\rangle$ & 4.1 & 4.2 & 4.4 & 4.8 & 5.2 \\
\hline
\end{tabular}

Table 5.6: Efficiencies of the dimuons isolation requirement for dimuons in NMSSM Monte Carlo sample with $m_{h_{1}}=100 \mathrm{GeV}$ and $m_{a_{1}}=2 \mathrm{GeV}$ and for background events $\epsilon_{B}^{d i-\mu}$, obtained using a background enriched sample with one dimuon and one orphan muon. We estimate the average expected 95\% C.L. upper limit on the rate of signal events before the isolation requirement is applied for several choices of isolation $I s o_{d i-\mu}$ cut values. Background rate estimate is based on the 3 events observed in this analysis in the side-band region with the default isolation requirement.

\subsection{Selection Efficiency for Benchmark Scenarios}

To illustrate the performance of the analysis to select possible signal, we use two benchmark models introduced earlier. Tables 5.7, 5.8 and 5.9 show event selection efficiencies obtained using the simulated signal events for these two benchmark scenarios. The tables demonstrate that analysis selections lead to a constant ratio $\alpha_{R E C O} / \alpha_{G E N}=0.74 \pm 0.02$ over all of the benchmark points used. This model independence of the ratio permits an easy estimate of the full event selection efficiency of this analysis for an arbitrary model predicting the same signature with two pairs of muons. The acceptance $\alpha_{G E N}$ can be calculated using a suitable event generator. The full efficiency $\alpha_{R E C O}$ is then calculated by multiplying $\alpha_{G E N}$ by the ratio $\alpha_{R E C O} / \alpha_{G E N}$. The full efficiency $\epsilon_{f u l l}$ could then be calculated by multiplying 


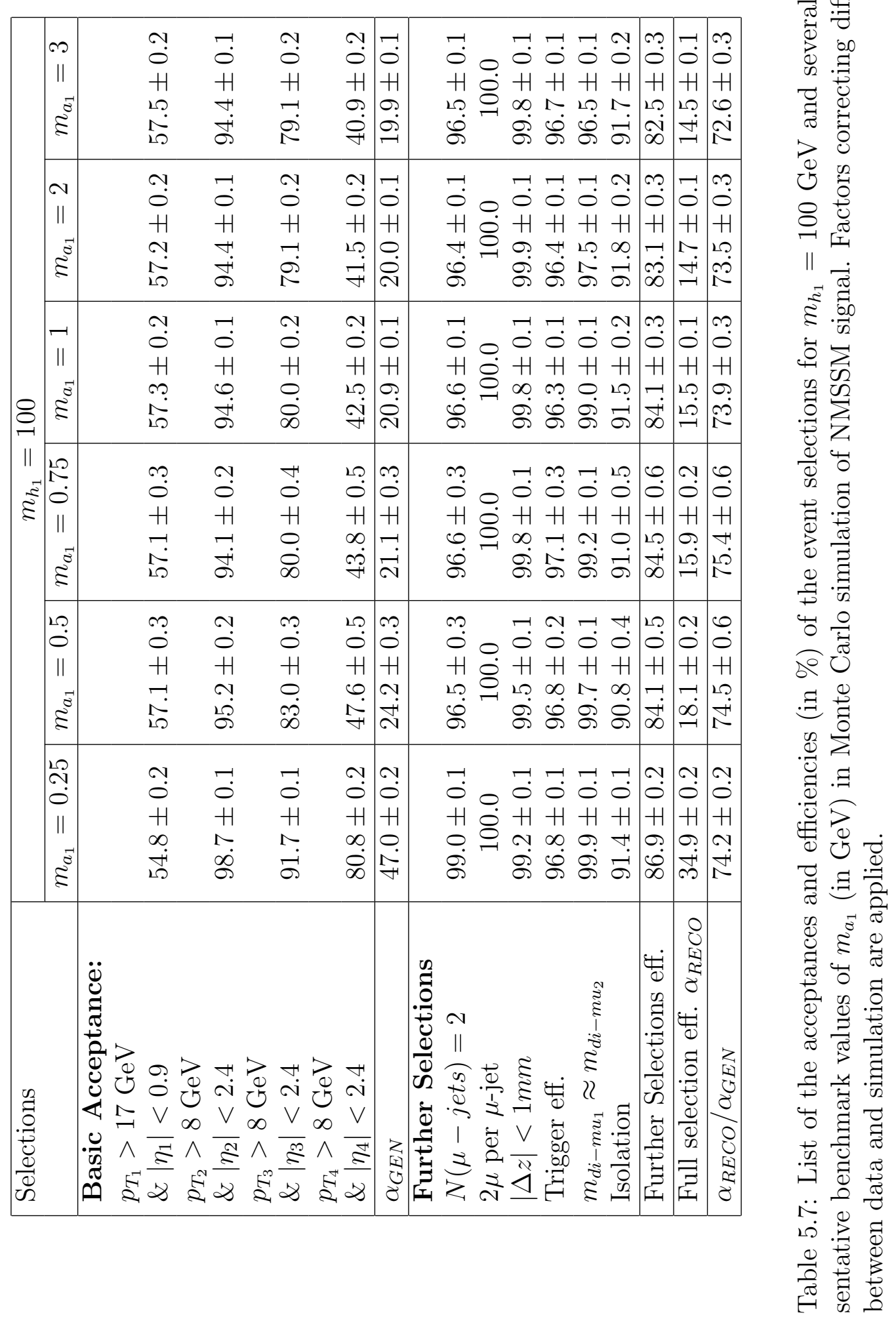




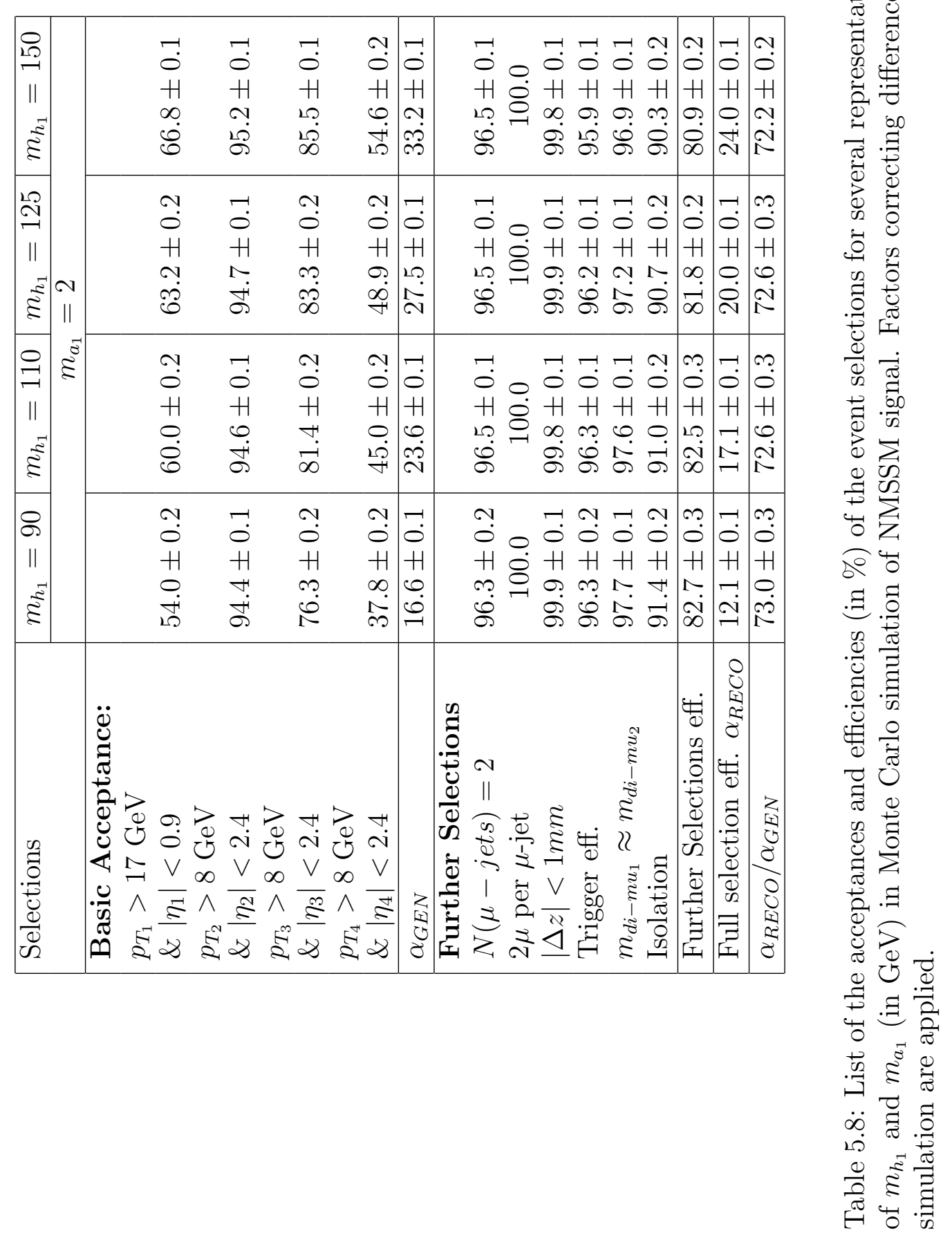




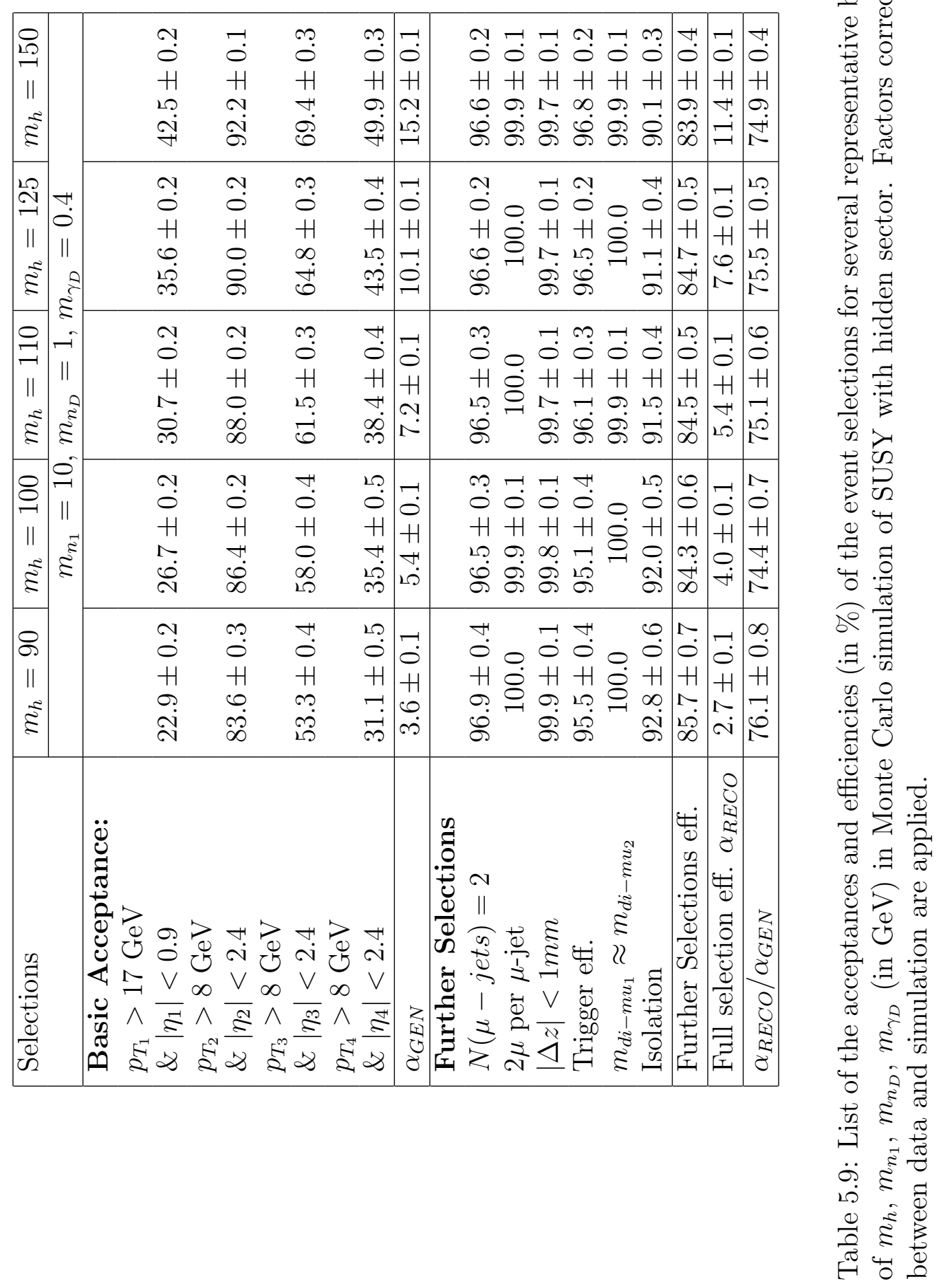


$\alpha_{R E C O}$ by $r=\epsilon_{\text {full }} / \epsilon_{R E C O}=0.91 \pm 0.07$, where $r$ is the scale factor that accounts for differences between data and simulation (see Sec. 5.5).

\subsection{Background Estimation}

The background contribution from the SM processes to a signature with two light dimuons after final selections of the analysis is dominated by the $b \bar{b}$ and direct $J / \psi J / \psi$ production. The contribution of other SM processes has been found to be negligible, e.g. the low mass Drell-Yan production is heavily suppressed by the requirement of two additional muons.

The leading part of the $b \bar{b}$ background contribution is dominated by events in which both $b$-quarks decay to pairs of muons $\left(\mu^{+} \mu^{-}+X\right)$ via either the double semileptonic decays or resonances, e.g. $\omega, \rho, \phi, J / \psi$. A smaller contribution comes from events with a true muon from semileptonic b-quark decays and a charged track misidentified as another muon due to an occasional incorrect assignment of some of the true muon's tracklets reconstructed in the muon system to a nearby track resulting in two muon candidates. The fraction of these cases decreases as the momentum of the two muons increases. Isolation imposed on dimuon candidates and a sufficiently low upper mass bound used to form muon-jets ensures that the two dimuons always come from two well separated $b$-jets.

The direct $J / \psi J / \psi$ contribution is estimated by normalizing the simulation predictions for $J / \psi J / \psi$ production to data in a few bins of invariant masses $m(J / \psi, J / \psi)$ and using them to estimate the rate of the direct $J / \psi J / \psi$ events satisfying all analysis selections, which yields an estimate of $0.3 \pm 0.3$ events (see Sec. 5.4.2).

\subsection{1 b $\bar{b}$ Background Shape}

In this analysis, the data in the signal region is fit to a sum of signal and background 2D templates. Modeling of the 2D signal shape is discussed in Sec. 5.2.2.4. 
This section describes modeling of the 2D shape for the background distribution.

Since both $b$-quarks decay independently of each other, the naïve assumption is that the $2 \mathrm{D}$ background shape can be modeled by a Cartesian product of two identical 1D shapes describing the dimuon distribution in $b$-jets that happen to have two muons in them. However, the shape of dimuon invariant mass depends on the muon transverse momentum selection criteria. In addition, it also depends on whether the dimuon is in the barrel or the endcap region due to different resolutions in these regions. Analysis selections require one of the dimuons to have at least one one muon with $p_{T}>17 \mathrm{GeV}$ in the barrel region $|\eta|<0.9$, while the muons in the other dimuon only required to have $p_{T}>8 \mathrm{GeV}$ and they can be either in the barrel or the endcap region. Thus, two dimuons can be divided into "high$p_{T}$ " and "low- $p_{T}$ " types depending on whether they contain a muon with $p_{T}>17$ $\mathrm{GeV}$ in the barrel region $|\eta|<0.9$. 1D shapes for each dimuon type are obtained separately: let's denote the $1 \mathrm{D}$ shapes for "high- $p_{T}$ " and "low- $p_{T}$ " types as $S_{17}\left(m_{\mu \mu}\right)$ and $S_{8}\left(m_{\mu \mu}\right)$, respectively (here "17" and "8" are related to muon $p_{T}$ thresholds). The 2D distribution of dimuon masses is denoted as $S\left(m_{1}, m_{2}\right)$, where $m_{1}=m_{\mu \mu_{1}}$ and $m_{2}=m_{\mu \mu_{2}}$. There can be only two combinations of dimuon types:

1. One dimuon is of the "high- $p_{T}$ " type and the other one is of the "low- $p_{T}$ " type. In this case the mass of the "high- $p_{T}$ " dimuon is taken as $m_{1}$ and the mass of the "low- $p_{T}$ " dimuon is taken as $m_{2}$ resulting in the following $2 \mathrm{D}$ mass template: $S_{17}(m 1) \times S_{8}(m 2)$.

2. Both dimuons are of the "high- $p_{T}$ " type. In this case the dimuons are randomly assigned to the $m_{1}$ or $m_{2}$ axis resulting in the following 2D mass template: $S_{17}(m 1) \times S_{17}(m 2)$.

If the rates of events entering the final distribution with the first and second com- 
bination of dimuons is $N_{8,17}$ and $N_{17,17}$, respectively, the normalized shape of the resulting $2 \mathrm{D}$ distribution is:

$S\left(m_{1}, m_{2}\right)=\frac{N_{17,8}}{N_{17,8}+N_{17,17}} \cdot\left[S_{17}\left(m_{1}\right) \times S_{8}\left(m_{2}\right)\right]+\frac{N_{17,17}}{N_{17,8}+N_{17,17}} \cdot\left[S_{17}\left(m_{1}\right) \times S_{17}\left(m_{2}\right)\right]$.

The 2D distribution can be factorized as:

$$
S\left(m_{1}, m_{2}\right)=S_{17}\left(m_{1}\right) \times\left[\frac{N_{17,8}}{N_{17,8}+N_{17,17}} \cdot S_{8}\left(m_{2}\right)+\frac{N_{17,17}}{N_{17,8}+N_{17,17}} \cdot S_{17}\left(m_{2}\right)\right] .
$$

Then, the 2D distribution is a Cartesian product of the mass distribution $S_{17}$ for "high- $p_{T}$ " dimuons and the proper mix $S_{m i x}$ of the mass distributions $S_{17}$ and $S_{8}$ for "high- $p_{T}$ " and "low- $p_{T}$ " types of dimuons:

$$
\begin{gathered}
S\left(m_{1}, m_{2}\right)=S_{17}\left(m_{1}\right) \times S_{m i x}\left(m_{2}\right) \\
S_{\text {mix }}(m)=\left[\frac{N_{17,8}}{N_{17,8}+N_{17,17}} \cdot S_{8}(m)+\frac{N_{17,17}}{N_{17,8}+N_{17,17}} \cdot S_{17}(m)\right] .
\end{gathered}
$$

which is used as the recipe in building the background 2D template of the analysis.

To construct the background templates $S_{17}$ and $S_{m i x}$, background-enriched events in the topology containing exactly one dimuon and one orphan muon are used. These events are collected using the same analysis selections except that the orphan muon is not required to have a pair. This data is heavily dominated by $b \bar{b}$ events and the topology and kinematics of these events are nearly identical to the events entering the sample with two dimuons.

To obtain the shape $S_{17}$, events where the dimuon is of the "high- $p_{T}$ " type and the orphan muon is only required to have $p_{T}>8 \mathrm{GeV}$ are used. The shape of the invariant mass distribution of the dimuons in the selected events is shown in Fig. 5.14 (left). 

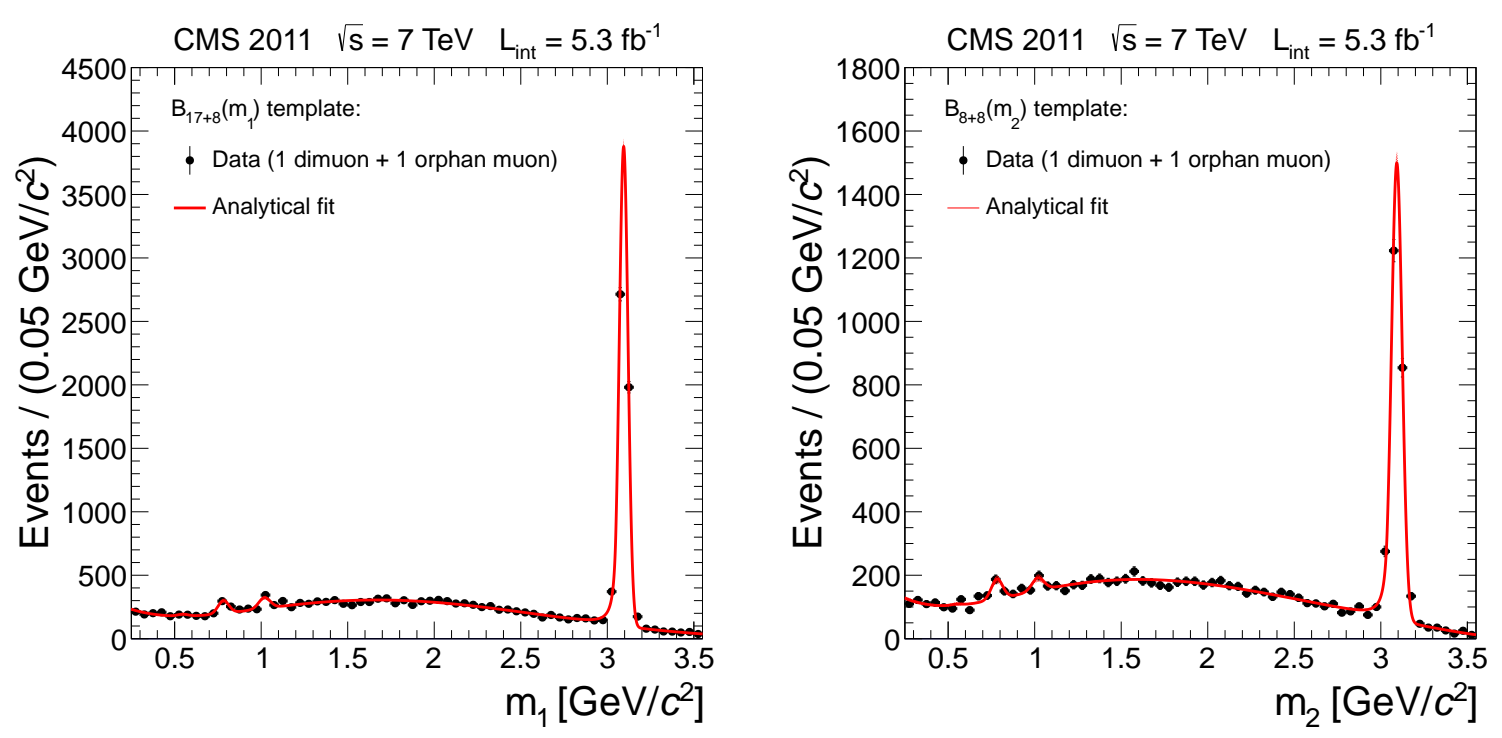

Figure 5.14: Background templates of the mass distribution $S_{17}$ for "high- $p_{T}$ " type of dimuons (left) and the proper mix $S_{m i x}$ of the mass distributions $S_{17}$ and $S_{8}$ for "high- $p_{T}$ " and "low- $p_{T}$ " types of dimuons (right).

To obtain the shape $S_{m i x}$, events where the orphan muon has $p_{T}>17 \mathrm{GeV}$ and is within $|\eta|<0.9$ and dimuon is of the "low- $p_{T}$ " type are used. The corresponding shape of the invariant mass distribution of the dimuons in the selected events is shown in Fig. 5.14 (right). This dimuon mass distribution contains proper fraction of both "high- $p_{T}$ " and "low- $p_{T}$ " dimuons. The final 2D template is a Cartesian product of distributions $S_{17}$ shown in Fig. 5.14 (left) and $S_{m i x}$ shown in Fig. 5.14 (right). The distribution in Fig. 5.14 (right) has a slightly enhanced "bulk" component and slightly broader shape of the resonances: these slight differences are expected as the dimuons in this case are on average less energetic and a fraction of them are in the endcap region.

For convenience of future use of the templates in the final fit, the $1 \mathrm{D}$ shapes shown in Fig. 5.14 (left) and (right) are fitted with a parametric analytical function 


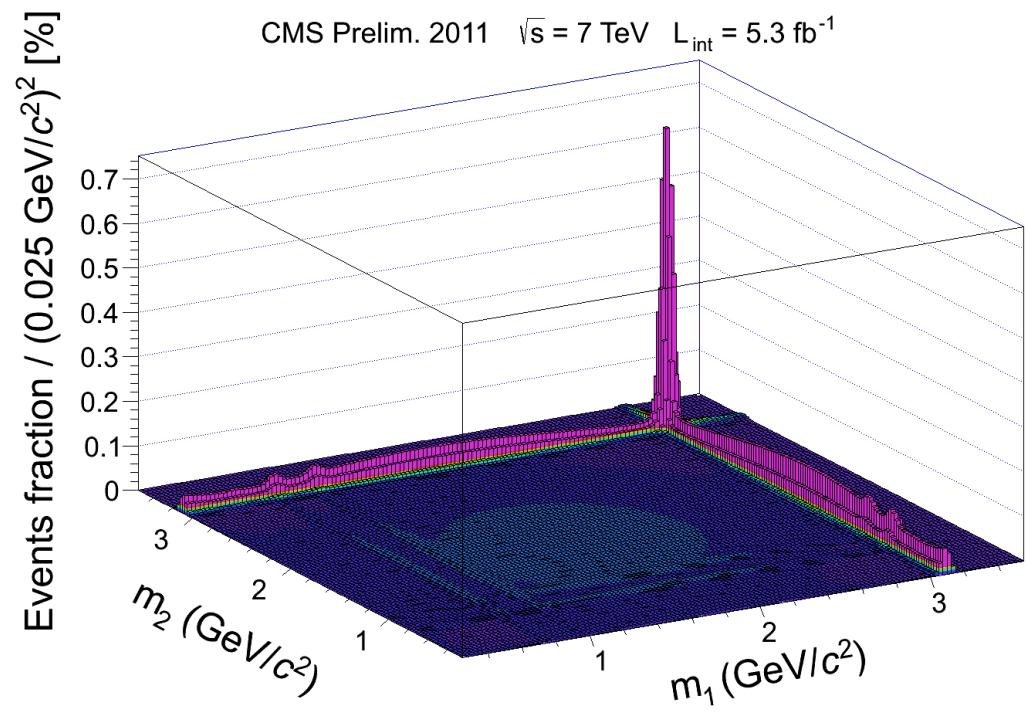

Figure 5.15: The 2D analytical template for distribution of the dimuon masses obtained using background-enriched data sample.

obtained using an extended unbinned likelihood fit:

$$
\begin{gathered}
B G\left(m ; p_{\eta}, p_{\omega}, p_{\phi}, p_{J / \psi}, \alpha_{J / \psi}, \sigma_{J / \psi}, p_{B}, p_{06}, \ldots, p_{66}\right)= \\
p_{\eta} G\left(m ; m_{0, \eta}, \sigma_{\eta}\right)+p_{\omega} G\left(m ; m_{0, \omega}, \sigma_{\omega}\right)+ \\
p_{\phi} G\left(m ; m_{0, \phi}, \sigma_{\phi}\right)+p_{J / \psi} C B\left(m ; m_{J / \psi}, \sigma_{J / \psi}, \alpha_{J / \psi}, n_{J / \psi}\right)+ \\
p_{B} B\left(m ; p_{06}, \ldots, p_{66}\right),
\end{gathered}
$$

where $\eta, \omega$, and $\phi$ resonances are parametrized using $G\left(m ; m_{0}, \sigma\right)$, a Gaussian normalized to a unit integral in the region $0.25<m<3.55 \mathrm{GeV}$. For each of these resonances, $m_{0}$ is fixed to the corresponding PDG mass and $\sigma$ (resolution) is fixed to the corresponding dimuon mass resolution obtained in Sec. 5.2.2.4. The $J / \psi$ resonance shape is parametrized with a Crystal Ball function, normalized to unit area in $0.25<m<3.55 \mathrm{GeV} . m_{J / \psi}$ is fixed to the PDG value, $\sigma_{J / \psi}, \alpha_{J / \psi}, n_{J / \psi}$ are obtained 
in Sec. 5.2.2.4, $\sigma_{J / \psi}$ is allowed to float in the fits. The bulk shape is described using $B\left(m ; p_{06}, \ldots, p_{66}\right)$, a series expansion in the Bernstein polynomial basis of power 6 . The $p_{\eta}, p_{\omega}, p_{\phi}, p_{J / \psi}, p_{B}$, and $B$ parameters are normalization factors for the resonances and the bulk component, respectively, and are obtained from the fit. Results of the fit are shown in Figs. 5.14 (left) and (right) as solid red lines.

The parameterized functional dependencies are then used to construct the 2D template shown in Fig. 5.15. The corresponding uncertainties in the fit parameters are saved including full correlation information to be used in the final analysis fit for a combination of signal and background.

To validate the obtained $2 \mathrm{D}$ shape, we return to the sample with two dimuons. The standard requirements have been applied to select these events, except the isolation requirement for dimuons has been removed in this check to obtain a sample with large statistics. The diagonal region is not considered to avoid accidental unblinding of the analysis in case of a signal present in data.

In Fig. 5.16 (top, left) the 1D projection of this 2D distribution on horizontal axis $m_{1}$ is shown (as black dots) and compared to the corresponding template $S_{17}$ (red line) obtained from background-enriched sample as described above. Also, in Fig. 5.16 (top, right) the $1 \mathrm{D}$ projection of the $2 \mathrm{D}$ distribution on vertical axis $m_{2}$ is shown (as black dots) and compared to the corresponding template $S_{m i x}$ (red line). To visualize the comparison, both $1 \mathrm{D}$ mass projections onto $m_{1}$ and $m_{2}$ axis are summed up (note that there are two entries per event) into one distribution for selected events as shown (black dots) in Fig. 5.16 (bottom) and compared to combined 1D template (red line) that comprises of both templates $S_{17}$ and $S_{m i x}$ stacked together. All 1D templates used for comparisons described above have been normalized to the number of selected events in the distributions obtained from data. All plots in Fig. 5.16 demonstrate good agreement between distributions obtained 

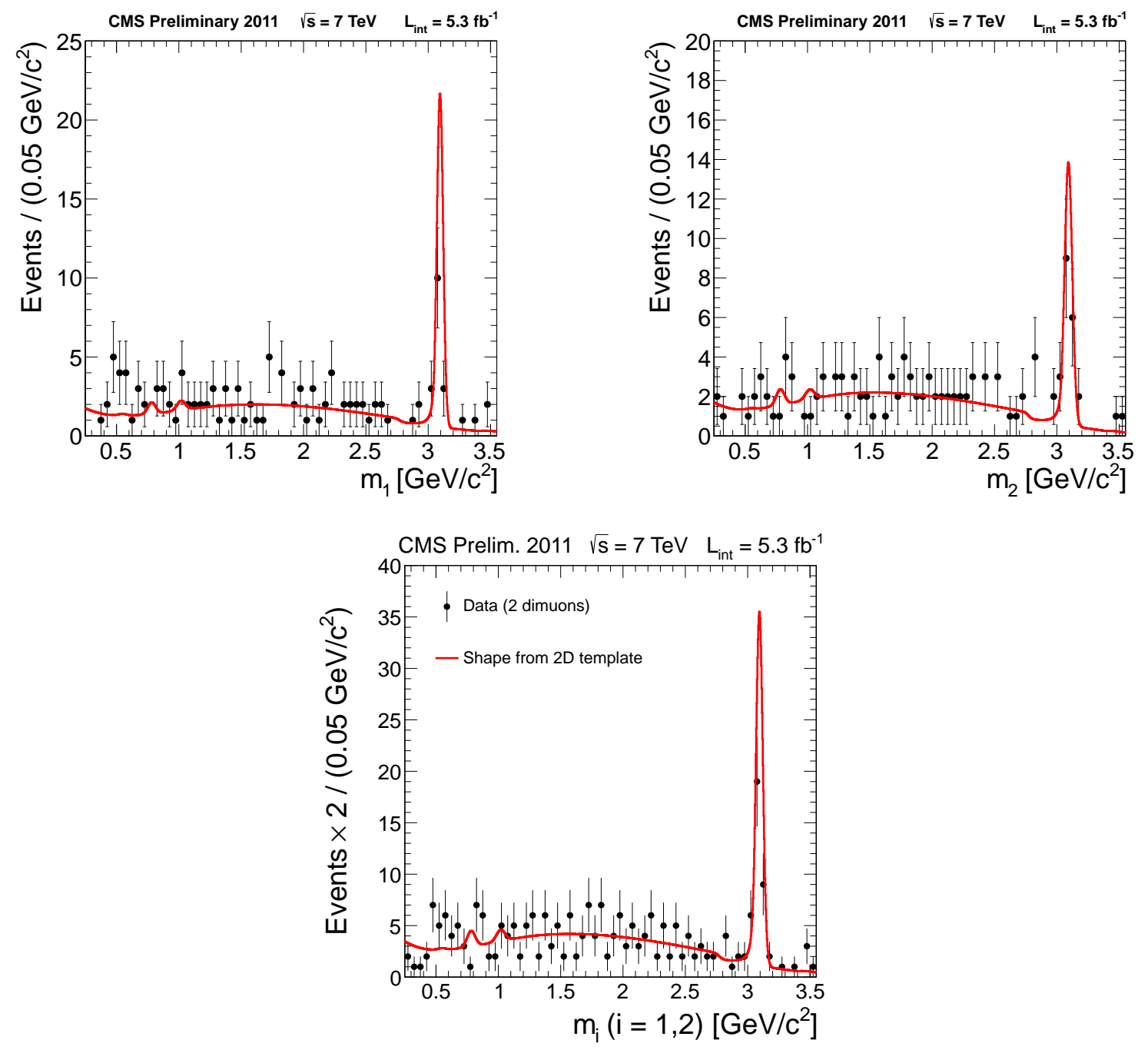

Figure 5.16: Top, left: distribution of the mass of the "high- $p_{T}$ " type of dimuons from events in the control region (two dimuons per event; no isolation requirement; only the off-diagonal region) compared to the analytical template $S_{17}$. Top, right: distribution of the mass of the proper mix of the "high- $p_{T}$ " and "low- $p_{T}$ " types of dimuons from events in the control region compared to the analytical template $S_{m i x}$. Bottom: distribution of the mass of all dimuons from events in the control region compared to the stacked analytical template $S_{17}+S_{m i x}$.

from events with two dimuons in the control region and the analytical templates obtained from the background-enriched events with one dimuon and one "orphan" muon. 
When all analysis procedures have been fixed and before the unblinding of the signal region, another validation comparison using the data sample of events with two dimuons has been performed. All signal selections have been applied except the dimuons are required to have isolation parameter between 3 and $8 \mathrm{GeV}$ (the loosely isolated but not well isolated dimuons). Four events have been found in the "off-diagonal" region. No data in the "diagonal corridor" have been observed (see Fig. 5.25 (right) on page 113 and Table 5.18 on page 115), which is consistent with the expectation based on the four events in the off-diagonal region. Using the $2 \mathrm{D}$ template, it has been estimated that the signal region contains approximately $18 \%$ of the total background (the other $82 \%$ are in the off-diagonal region); out of the $18 \%$ in the diagonal region approximately $7 \%$ are in the vicinity of $J / \psi$ resonance. Simple estimation shows that the expected background rate in the signal region is approximately $(4 \pm 2) \times 0.18 / 0.82=0.88 \pm 0.44$ events, which is consistent with no observed data.

\subsubsection{Prompt Double $J / \psi$ Production}

The $b \bar{b}$ background estimation procedure described in the previous section accounts for $J / \psi$ resonances produced in $b \bar{b}$ decays, but doesn't take into account prompt double $J / \psi$ production. In this section, we provide details of estimation of the background contribution from such production.

We estimate the rate of the prompt $J / \psi J / \psi$ events using the Monte Carlo simulation of the prompt double $J / \psi$ production through double parton scattering corrected using the CMS measurement [143] of the differential cross section $d \sigma(p p \rightarrow$ $J / \psi J / \psi) / d M$, where $M$ is the invariant mass of the two $J / \psi$ 's in the event. Since the MC sample used in Ref. [143]) has too low equivalent integrated luminosity to provide adequate statistics to estimate the rate after applying the analysis selection 
requirements, we have generated a new sample applying higher $p_{\mathrm{T}}$ thresholds on $J / \psi$ 's. We refer to these samples as "low- $p_{T}$ " and "high- $p_{T}$ " samples, respectively. The "high- $p_{T}$ " sample has allowed us to achieve reasonable statistics after the analysis selections applied. The samples have been cross-normalized using the region where both samples overlap.

The "low- $p_{T}$ " sample contains 223,000 events with directly produced pair of $J / \psi$ 's satisfying the two following selections:

- two $J / \psi$ with $p_{\mathrm{T}}>3 \mathrm{GeV}$ and $|\eta|<2.5$;

- two muons with $p_{\mathrm{T}}>2.5 \mathrm{GeV}$ and $|\eta|<2.5$.

Distribution of events in the "low- $p_{T}$ " sample versus invariant mass of the two $J / \psi$ 's is shown in Fig. 5.17. The measurement of $d \sigma(p p \rightarrow J / \psi J / \psi) / d M$ has been performed at the CMS in several bins of $M$ as shown in Table 5.10 (see details in Ref. [143]). To obtain the equivalent luminosity of the "low- $p_{T}$ " sample, we use the differential cross-section $d \sigma / d M^{\mathrm{CMS} \text { Data }}$ measured in the same bins of $M$ and the number of generated events $N_{M C}^{l o w-p_{T}}$ in the same mass ranges. If the spectra in the data and in the simulation were to be identical, the ratio $N_{M C}^{l o w-p_{T}} /\left(d \sigma / d M^{\mathrm{CMS} \text { Data }}\right)$ would provide the equivalent luminosity $L_{M C}^{\text {low- } p_{T}}$ of the "low- $p_{T}$ " sample in any bin of $M$. Because the spectra are apparently not identical, the calculated ratio is effectively equal to $L_{M C}^{l o w-p_{T}} / K_{c o r}$, where $K_{c o r}$ is a scale factor, which needs to be applied to the simulation to bring it in the agreement with the data.

We have applied the analysis selection requirements to the "low- $p_{T}$ " sample of the simulated prompt $J / \psi J / \psi$ events, but zero events have survived the selection. So, the "low- $p_{T}$ " sample has too low statistics to obtain a prediction for the background contribution in our analysis. Therefore, we have simulated a new, "high- $p_{T}$ ", sample of the prompt $J / \psi J / \psi$ events, using the settings identical to the "low- $p_{T}$ " one, except 


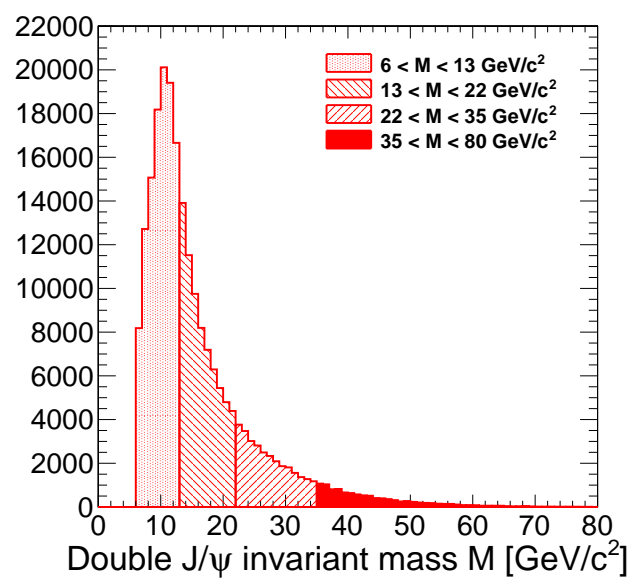

Figure 5.17: The distribution of the invariant mass $M$ of the two $J / \psi$ 's with $p_{T}^{J / \psi_{1,2}}>3$ GeV in the "low- $p_{T}$ " sample.

\begin{tabular}{|c|c|c|c|c|c|}
\hline Bin & $\begin{array}{c}\left(M_{1}-M_{2}\right) \\
\mathrm{GeV}\end{array}$ & $\begin{array}{c}d \sigma / d M^{\mathrm{CMS} \text { Data }} \\
\mathrm{nb} / \mathrm{GeV}\end{array}$ & $\begin{array}{c}\int_{M_{1}}^{M_{2}} d \sigma / d M^{\mathrm{CMS} \text { Data }} \times d M \\
\mathrm{nb}\end{array}$ & $N_{M C}^{\text {low- } p_{T}}$ & $\begin{array}{c}L_{M C}^{\text {low-p }} / K_{\text {cor }} \\
\mathrm{fb}^{-1}\end{array}$ \\
\hline 1 & $(6-13)$ & $0.064 \pm 0.005$ & $0.45 \pm 0.03$ & 110,339 & 0.25 \\
2 & $(13-22)$ & $0.007 \pm 0.001$ & $0.06 \pm 0.01$ & 71,495 & 1.19 \\
3 & $(22-35)$ & $0.005 \pm 0.001$ & $0.07 \pm 0.01$ & 29,042 & 0.41 \\
4 & $(35-80)$ & $0.001 \pm 0.001$ & $0.05 \pm 0.05$ & 10,788 & 0.22 \\
\hline
\end{tabular}

Table 5.10: The ratio of the equivalent integrated luminosity $L_{M C}^{\text {low- } p_{T}}$ of the "low$p_{T}$ " sample of the simulated prompt $J / \psi J / \psi$ events and the correction factor $K_{c o r}$ accounting for the apparent difference in the spectra of prompt double $J / \psi$ production in data and simulation.

that we filter events by requiring each of the two $J / \psi$ 's have $p_{T}>10 \mathrm{GeV}$. This allowed us to obtain a sample that has a reasonable total number of events and also has a meaningful number of events surviving the analysis selections. The "high- $p_{T}$ " sample has been normalized with respect to the "low- $p_{T}$ " sample using numbers of events with $p_{T}^{J / \psi_{1,2}}>10 \mathrm{GeV}$ in different bins of $M$ as shown in Table 5.11. The corresponding distributions are shown in Fig. 5.18. 

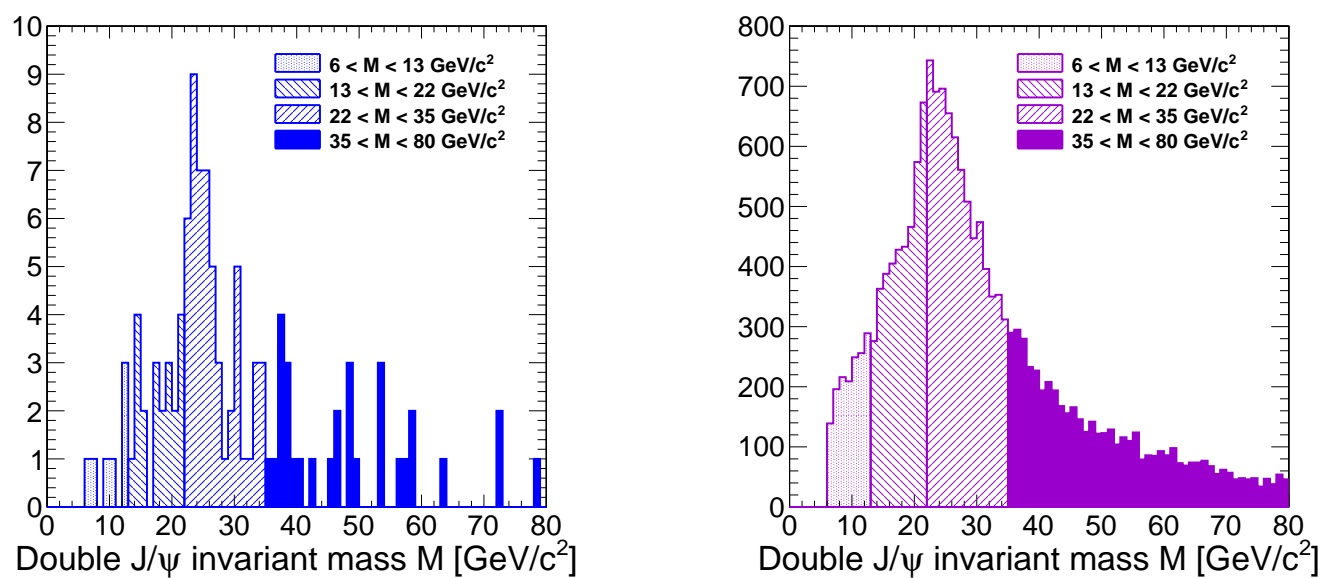

Figure 5.18: The distribution of the invariant mass $M$ of the two $J / \psi$ 's with $p_{T}^{J / \psi_{1,2}}>$ $10 \mathrm{GeV}$ in the "low- $p_{T}$ " (left) and in the "high- $p_{T}$ " (right) samples.

Even with the much larger number of events in the "high- $p_{T}$ " sample, there are only $N_{\text {Pass }}^{h i g h-p_{T}}=3$ events that pass the kinematic and geometrical acceptance selections at the generator level for our analysis. We use the ratio of $\alpha_{R E C O} / \alpha_{G E N}=0.74$ to predict the expected number of events passing our analysis selections using reconstructed objects and normalize it to the integrated luminosity of $L_{\text {Data }}=5.3 \mathrm{fb}^{-1}$, which corresponds to the dataset used in our analysis:

$$
N_{\text {Expected }}=N_{\text {Pass }}^{h i g h-p_{T}} \times R_{\text {Gen-to-Reco }} \times L_{\text {Data }} \times\left(L_{M C}^{h i g h-p_{T}} / L_{M C}^{l o w-p_{T}}\right) \times\left(L_{M C}^{l o w-p_{T}} / K_{\text {cor }}\right),
$$

where the expressions in the parentheses are taken from the Tables 5.10 and 5.11. Note that this estimate for the number of prompt double $J / \psi$ events in our analysis includes the simulation-to-data correction factor $K_{\text {cor }}$ introduced above. Since, $K_{c o r}$ is different depending which bin in $M$ has been used to normalize the "low- $p_{T}$ " sample, we calculate the expected number of events in all four bins of $M$. Finally, we take $0.3 \pm 0.3$ as the estimate of the prompt double $J / \psi$ contribution to the signal 


\begin{tabular}{|c|c|c|c|c|}
\hline Bin & $\left(M_{1}-M_{2}\right)$ & $\begin{array}{c}N_{M C}^{\text {low- }} \\
\left(p_{\mathrm{T}}^{J / \psi_{1,2}}>10 \mathrm{GeV}\right)\end{array}$ & $\begin{array}{c}N_{M C}^{\text {high-p }} \\
\left(p_{\mathrm{T}}^{J / \psi_{1,2}}>10 \mathrm{GeV}\right)\end{array}$ & $L_{M C}^{\text {high-p }} / L_{M C}^{\text {low- }}$ \\
\hline 1 & $(6-13)$ & 7 & 1,554 & 222.0 \\
2 & $(13-22)$ & 21 & 4,006 & 191.3 \\
3 & $(22-35)$ & 53 & 6,801 & 128.3 \\
4 & $(35-80)$ & 30 & 5,195 & 173.6 \\
\hline
\end{tabular}

Table 5.11: The equivalent integrated luminosity of the "high- $p_{T}$ " sample calculated relative to the equivalent integrated luminosity of the "low- $p_{T}$ " sample in different bins of $M$ using the region with both $J / \psi$ 's having $p_{T}>10 \mathrm{GeV}$. The card used in the "high- $p_{T}$ " simulation is identical to the one in the "low- $p_{T}$ " simulation.

region of our analysis since it covers the spread of the predictions obtained for each bin in $M$ (see Table 5.12).

\begin{tabular}{|c|c|c|c|}
\hline Bin & $\left(M_{1}-M_{2}\right)$ & $N_{\text {pass }}^{\text {high }-p_{T}}$ & $N_{\text {Expected }}$ \\
\hline 1 & $(6-13)$ & & $0.22 \pm 0.15$ \\
2 & $(13-22)$ & & $0.05 \pm 0.03$ \\
3 & $(22-35)$ & 3 & $0.22 \pm 0.13$ \\
4 & $(35-80)$ & & $0.3 \pm 0.3$ \\
\hline
\end{tabular}

Table 5.12: The expected number of prompt double $J / \psi$ events passing selections of this analysis extrapolating the CMS measurements of the prompt double $\mathrm{J} / \psi$ production in four bins of $M$. The difference in predictions stems from the difference in the shape of the differential cross-section in data and simulation. The spread is taken as a systematic uncertainty on the expected number of the prompt double $\mathrm{J} / \psi$ events in this analysis.

\subsection{Systematic Uncertainties}

Table 5.13 lists sources of systematic uncertainties that have been accounted in the procedure for setting upper limits on the signal in the analysis. 


\begin{tabular}{|l|l|}
\hline Source of uncertainties & Error, \% \\
\hline Integrated luminosity & $2.2 \%$ \\
\hline Muon HLT & $1.5 \%$ \\
\hline Muon ID & $4 \times 1.4 \%$ \\
\hline Muon tracking & $4 \times 0.2 \%$ \\
\hline Overlapping in Tracker & $2 \times 1.2 \%$ \\
\hline Overlapping in Muon System & $2 \times 1.3 \%$ \\
\hline Dimuons mass consistency & $1.5 \%$ \\
\hline PDF $+\alpha_{s}$ & $3 \%$ \\
\hline Total & $8.0 \%$ \\
\hline
\end{tabular}

Table 5.13: Summary of the magnitude of systematic uncertainties.

The following subsections provide details about each of these sources of systematic uncertainties.

\subsubsection{Sources of Systematic Uncertainties}

\subsubsection{Luminosity Measurement}

The uncertainty on the luminosity measurement is standard and is estimated as $2.2 \%[144]$.

\subsubsection{Muon Trigger}

The efficiency of muon trigger (HLT_Mu17_Mu8) is evaluated by performing the measurement of the trigger efficiency per leg using the Tag and Probe technique separately for the Mu17 and Mu8 legs of the dimuon trigger as described in Sec. 5.5.2. The efficiencies have been compared with the simulation predictions to derive a scale factor for per leg efficiency measured in simulation. To account for the presence of four muons, the trigger efficiency in simulation has been corrected for combinatorial effects, taking into account the reduced trigger efficiency per muon when two muons 
are nearby* ${ }^{*}$ The scale factor for per-event efficiency is $0.984 \pm 0.015$, where $1.5 \%$ becomes the systematic uncertainty.

\subsubsection{Muon Identification}

The efficiency of muon identification has been measured using the Tag and Probe technique to derive a scale factor for the per leg efficiency of muon identification as described in Sec. 5.5.2. We assign a systematic uncertainty of $1.4 \%$ for each of the four muons in the event, which accounts for small variations in the scale factor over the $p_{T}$ range and also includes a recommended by the Muon POG add-on uncertainty to account for the deviations in the scale factor versus pseudorapidity. For tracking efficiency we have followed the study in [145] and have found the scale factor to be 1.002 with systematic uncertainty of $0.2 \%$ per muon.

\subsubsection{Muon Trajectory Overlaps in the Muon System}

To account for correlated effects of muon reconstruction when two muons are close to each other in the muon system, we use the results of the studies in [140], where the systematic uncertainty associated with this effect has been conservatively evaluated by comparing the difference per leg efficiency for cases with and without a nearby muon present (so called crossing and non-crossing topologies), which yielded a systematic uncertainty of $1.3 \%$ per muon in the endcap region only. No such effect have been observed in the barrel region. As in our events there is at most one dimuon in the endcap region, we assign the uncertainty of $2.6 \%$ per event to account for the uncertainties in reconstructing muons with overlapping trajectories in the muon system. There is only one relevant difference between muon identifications requirements in this analysis and in [140], which is the requirement on the number

\footnotetext{
*The trigger nearly always finds at least one of the two muons, but has a substantial probability of not reconstructing the other muon.
} 
of muon stubs per muon (at least two in this analysis, at least three in [140]). As the muon reconstruction is unchanged, it is only a matter of what muons each analysis picks out of the pool of reconstructed muons. As this analysis has looser requirement, the importance of the effect of nearby muons only becomes smaller (we find all muons that would have been found by [140] and more, which makes the loss of efficiency due to overlaps smaller). Therefore, the uncertainty of $2.6 \%$ per event is a conservative estimate of the effects related to overlaps in the muon system.

\subsubsection{Dimuon Isolation}

The efficiency of the isolation requirement that is applied to dimuons has been corrected using a scale factor obtained with data and MC samples of $Z \rightarrow \mu \mu$ events. In both cases the isolation is driven by the underlying event activity. The results are again based on the Tag and Probe method and the details are described in Sec. 5.5.2.

The derived scale factor between data and $\mathrm{MC}$ is $f_{\text {Iso }}^{d i-\mu}=0.988$ and the uncertainty of the measurement is negligible and therefore not included in the table of systematic uncertainties.

\subsubsection{Pile-Up Events}

Effects related to the accuracy of simulating the number of pile-up interactions in the signal MC has been evaluated comparing full selection efficiencies including all detector effects for the NMSSM sample simulated with two different distributions of the number of reconstructed vertices. The first distribution (default for all MC samples in this analysis) has been exactly matched to the reference distribution obtained for runs corresponding to our dataset, using unbiased distribution measured by the luminosity group. The second one is an initial estimate of the distribution of the number of reconstructed vertexes before data collection in second half of year 2011, it has been used by CMS for centralized production of MC samples. 
The difference between two distributions provides a conservative estimate of changes in pile-up conditions during 2011. The change in full selection efficiency is only $0.5 \%$. We therefore conclude that the full selection efficiency including all detector effects is very weakly sensitive to broadly varied pile-up conditions and the systematic uncertainty is negligible.

\subsubsection{Muon Trajectory Overlaps in the Tracker}

In [140], a potential effect related to tracking has been identified as low mass muon pairs at sufficiently high momentum can stay very close in the tracking volume leading to reduced efficiency of reconstructing both tracks in the dimuon, due to merging of tracker hits into clusters. The assumption is that if the track hit size is larger in data than in the simulation by $20 \%$, merging will be happening more frequently in data leading to lower efficiency to reconstruct both muons. As varying hit resolutions in simulation is a complicated endeavor, an easier but equivalent solution is to vary the momentum of the dimuons by the same $20 \%$, which makes trajectories $20 \%$ closer and comparing efficiencies. We compared these efficiencies for reconstructed dimuons with transverse momenta from 16 to $60 \mathrm{GeV}$ (typical in the current analysis) using Fig. 7 in [140]. As a result, the corresponding systematic uncertainty is estimated as $1.2 \%$ per dimuon.

\subsubsection{Modeling of the Signal Shape}

The accuracy in the efficiency of the dimuon mass compatibility requirement is driven by the accuracy with which simulation describes the radiative tail in the signal shape. The size of the signal region is much larger than the detector resolution. Therefore, the inefficiency and, thus, potential systematic uncertainty is small. To estimate the effect, the parameters of the Crystal Ball fit to $J / \psi$ events in data (we use the background enriched sample containing one dimuon and one orphan 
muon) have been deviated within the fit statistical uncertainties on the parameters. Assuming that the distribution of signal events is similar to the obtained template, the corresponding deviation $1.5 \%$ in the acceptance within signal region has been calculated and assigned as systematic uncertainty of this effect.

\subsubsection{Theoretical Uncertainties}

Theoretical systematic error on the signal acceptance come from the uncertainties in $\mathrm{PDF}+\alpha_{s}$ and from the uncertainties evaluated by varying QCD renormalization and factorization scales $\left(\mu_{R}\right.$ and $\left.\mu_{F}\right)$. As it has been found in [146, 147] by using MCFM and varying $\mu_{R}$ and $\mu_{F}$ by a factor of two up and down the acceptance errors are very small and, therefore, can be neglected.

Additionally a systematic error of $3 \%$ in the acceptance is included to account for uncertainties in $\mathrm{PDF}+\alpha_{s}$ by varying parametrization within the CTEQ6.6 [18] family, and comparing the central values of CTEQ6.6L with NNPDF2.0 [19], and MSTW2008 [20] sets.

To exclude specific types of the NMSSM models, one relies on the knowledge of the predicted yield of the $h \rightarrow a a \rightarrow 4 \mu$ events. Therefore it is important to include the corresponding uncertainties in the uncertainty on Higgs cross section and branching fraction $\operatorname{Br}(h \rightarrow a a \rightarrow 4 \mu)$. Systematic errors on the signal total cross section for each production mechanism and for all Higgs boson masses have been calculated and are available elsewhere [148]. They come from PDF $+\alpha_{s}$ systematic errors and varying QCD renormalization and factorization scales. The uncertainties are treated as uncorrelated and total systematic uncertainty of $3 \%$ (Higgs boson mass independent) is assigned. The uncertainty on the branching fraction is taken to be $2 \%$ and assumed to be Higgs boson mass independent. 


\subsubsection{Tag and Probe Studies}

The tag and probe (T\&P) study of the muon efficiencies has been performed to account for the difference between $\mathrm{MC}$ simulation and real data. All correction factors that have been obtained from the study are summarized in Table 5.14.

\begin{tabular}{|l|l|}
\hline Source of correction & Factor \\
\hline$f_{\text {MuonID }}^{\mu}$ & 0.987 \\
\hline$f_{H L T}^{\text {evt }}$ & 0.984 \\
\hline$f_{\text {Iso }}^{d i-\mu}$ & 0.988 \\
\hline$f_{z-\text { vertex }}^{d i-\mu}$ & 1.00 \\
\hline Total & 0.911 \\
\hline
\end{tabular}

Table 5.14: Summary of the correction (simulation to data) factors.

\subsubsection{Muon Identification Scale Factor}

In the study of the analysis muon identification algorithm with respect to muon tracking efficiency in the Silicon tracker, a tag muon is defined as the following:

- identified as a GlobalMuon of the collection muons

- passes muon tight selection (GlobalMuonPromptTight)

- $p_{T}>40 \mathrm{GeV}$ and $|\eta|<2.4$

- matches to a trigger object in either HLT_Mu40_v* or HLT_Mu40_eta2p1_v* path.

A probe is defined as the following:

- identified as a silicon tracker track of the collection generalTracks

- $p_{T}>8 \mathrm{GeV}$ and $|\eta|<2.4$. 

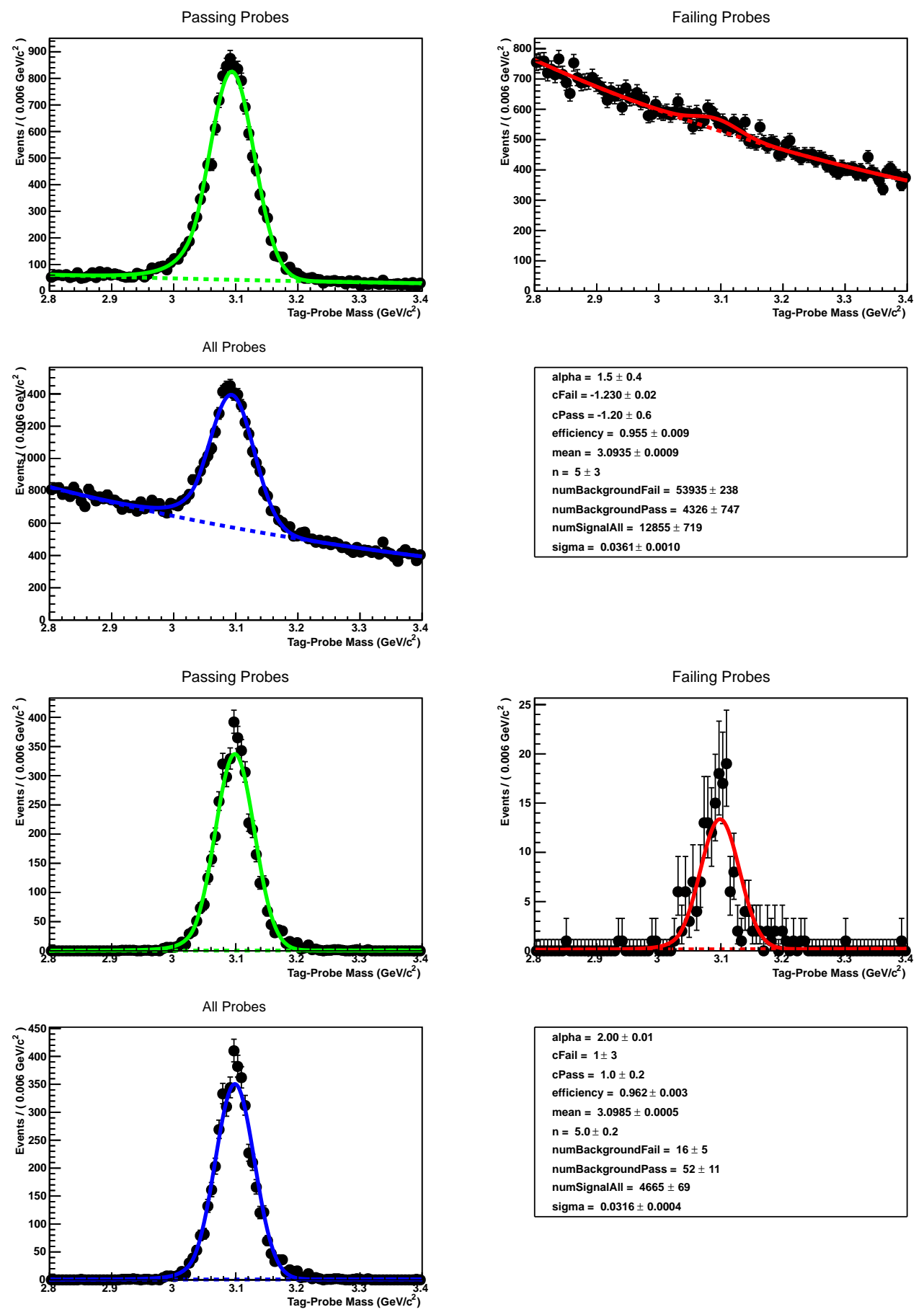

Figure 5.19: Distributions of dimuon masses consistent with $J / \psi$ mass (from 2.8 to $3.4 \mathrm{GeV}$ ) containing passing, failing, and all probe muons with $p_{T}$ from 8 to $20 \mathrm{GeV}$ in tag and probe study of the analysis muon identification efficiency. Top four: data; bottom four: MC. 

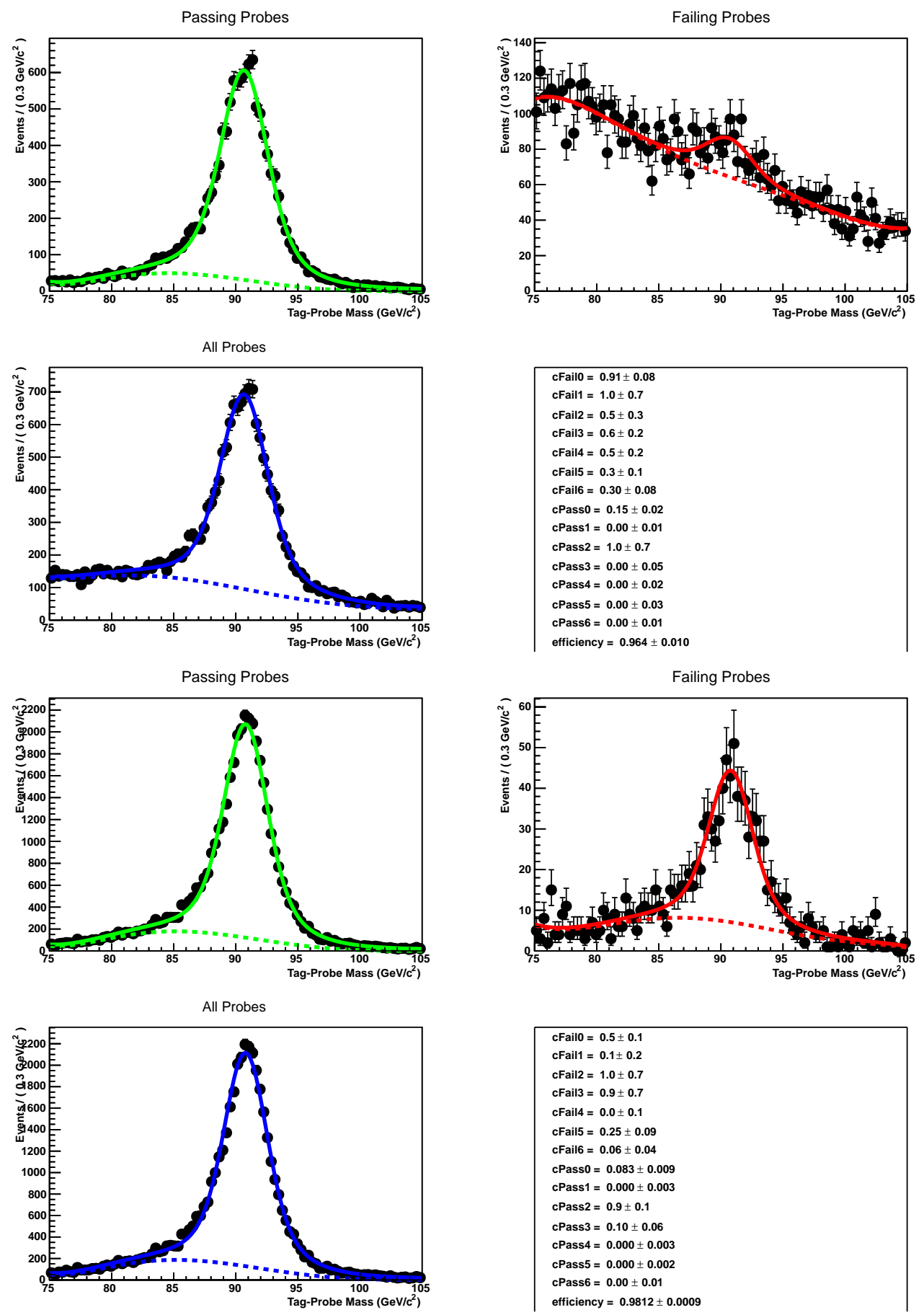

Figure 5.20: Distributions of dimuon masses consistent with $Z$ mass from 75 to $105 \mathrm{GeV}$ ) containing passing, failing, and all probe muons with $p_{T}$ from 20 to $40 \mathrm{GeV}$ in tag and probe study of the analysis muon identification efficiency. Top four: data; bottom four: MC. 

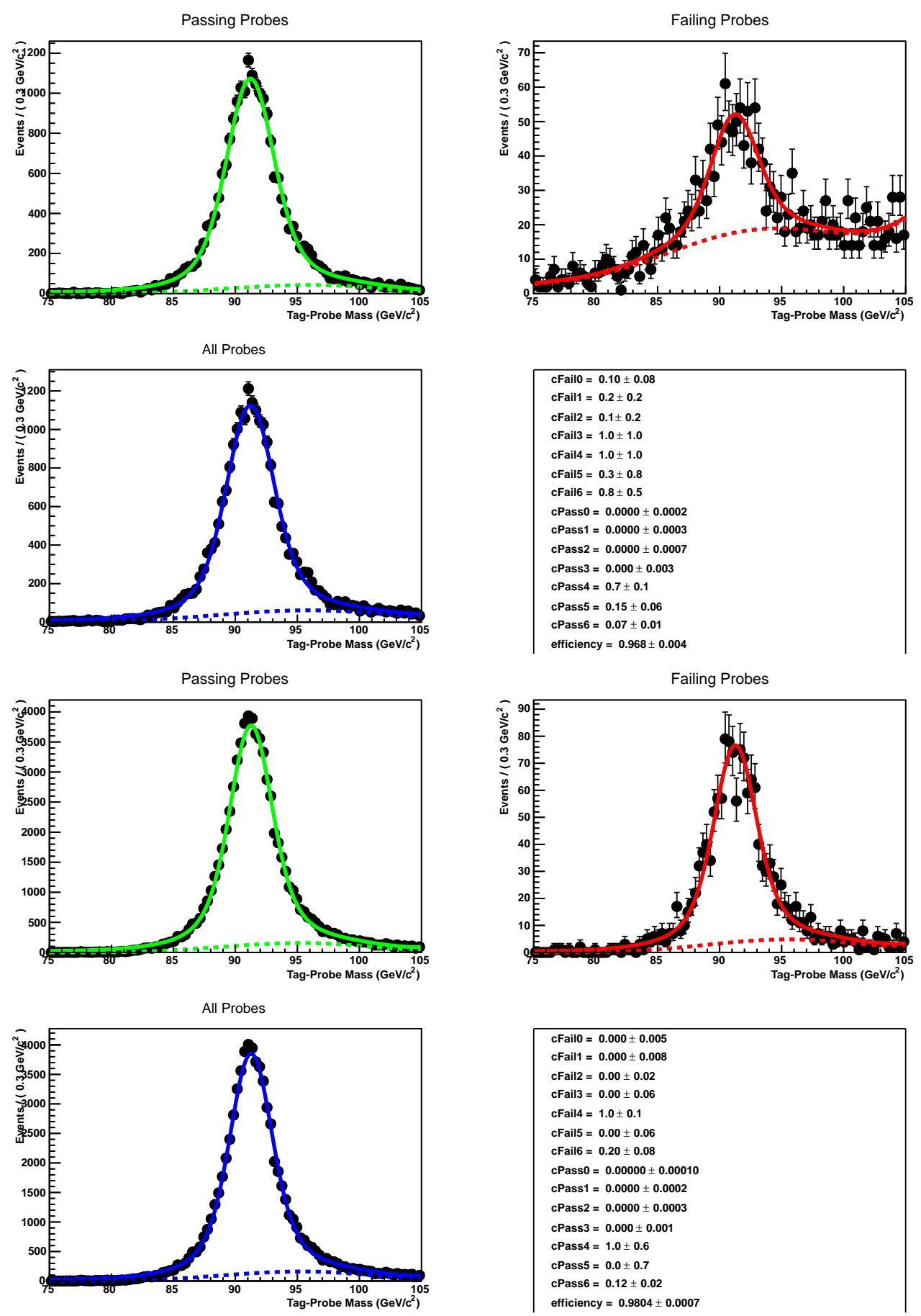

Figure 5.21: Distributions of dimuon masses consistent with $Z$ mass (from 75 to $105 \mathrm{GeV}$ ) containing passing, failing, and all probe muons with $p_{T}$ from 40 to $60 \mathrm{GeV}$ in tag and probe study of the analysis muon identification efficiency. Top four: data; bottom four: MC. 
If a muon has the same inner track as a probe and it passes the analysis muon identification requirements then the probe is defined as passing probe, otherwise the probe is failing. In addition, the study has been performed in three non-overlapping bins of the probe muons transverse momentum: 8-20 GeV, 20-40 GeV and 40-60 GeV. The results of this study are presented in Table 5.15. All standard output of T\&P application are shown in Fig. 5.19, 5.20 and 5.21.

\begin{tabular}{|c|c|c|c|}
\hline Probe muon & \multicolumn{2}{|c|}{ Efficiencies } & $\begin{array}{c}\text { Scale factor } \\
p_{T} \text { range, GeV }\end{array}$ \\
\cline { 2 - 4 } & in data $E_{\text {MuonID,Data }}$ & in MC $E_{\text {MuonID,MC }}$ & $f_{\text {MuonID }}=\frac{E_{\text {MuonID,Data }}}{E_{\text {MuonID,MC }}}$ \\
\hline $8-20$ & $0.955 \pm 0.009$ & $0.962 \pm 0.003$ & $0.993 \pm 0.009$ \\
\hline $20-40$ & $0.964 \pm 0.010$ & $0.9812 \pm 0.0009$ & $0.982 \pm 0.010$ \\
\hline $40-60$ & $0.968 \pm 0.004$ & $0.9804 \pm 0.0007$ & $0.987 \pm 0.004$ \\
\hline
\end{tabular}

Table 5.15: Results of the T\&P study of the analysis muon identification algorithm with respect to muon tracking efficiency in the Silicon tracker in data and MC samples.

The resulting scale factor between data and MC is set to

$$
f_{\text {MuonID }}=0.987 \pm 0.010(\text { stat }) \pm 0.010(\text { syst }),
$$

where the statistical uncertainty is taken from the bin with lowest statistics available (with probe muon $p_{T}$ from 20 to $40 \mathrm{GeV}$ ), the values in other two bins with probe muon $p_{T}$ in the ranges $8-20 \mathrm{GeV}$ and $40-60 \mathrm{GeV}$ are within this uncertainty. Systematic uncertainty is assigned to the scale factor to include up to $1 \%$ deviations of the efficiencies in different ranges of pseudorapidity of tag and probe muons (e.g. barrel-barrel, barrel-endcap or endcap-endcap) as it has been studied by Muon 
$\mathrm{POG}^{\dagger}$

\subsubsection{Muon Trigger Efficiency Scale Factor}

To validate the performance of the HLT_Mu17_Mu8 trigger, we first measure trigger efficiency per leg for each of the two legs in the trigger separately. The per leg efficiency for HLT_Mu8 and HLT_Mu17 is measured with respect to well identified muons passing offline muon identification selections used in this analysis. The sample has been selected using the single muon trigger. The tag muon definition has been the same as for the muon ID efficiency measurement described above. A high- $p_{T}$ probe is a muon passing the analysis muon identification requirements with $p_{T}>17 \mathrm{GeV}$ and $|\eta|<0.9$. A low- $p_{T}$ probe is a muon passing the analysis muon identification requirements with $p_{T}>8 \mathrm{GeV}$ and $|\eta|<2.4$. Probes matched to a trigger objects in HLT_Mu17_Mu8 path become passing probes. Figures 5.22 and 5.23 correspondingly show standard output of the T\&P application for high- $p_{T}$ and low- $p_{T}$ muons. As expected, the per leg efficiencies for $\operatorname{high}-p_{T}$ and low- $p_{T}$ muons are very similar independent of muon $p_{T}$ with the average per leg efficiencies of $\epsilon_{H L T}^{s i m}=96.7 \%$ and $\epsilon_{H L T}^{\text {data }}=95.2 \%$ with the uncertainties of a fraction of a percent.

The next step is to propagate the per leg scale factors for muon trigger efficiencies into efficiencies per event, in which there are four muons but the trigger only requires two. In simulation, one can measure the per leg efficiencies and per event efficiency, but it does not tell one how exactly the convolution happens, so we need to come up with a reasonable way to measure it. The main challenge comes from the correlation of muon reconstruction in the trigger when there are two true trigger quality muons nearby. The trigger highly efficiently finds the higher momentum muon in the pair, but the probability of finding two muons is lower than the product of two muon effi-

\footnotetext{
${ }^{\dagger}$ see report "Luminosity study with $Z \rightarrow \mu \mu$ " by Stoyan Stoynev at Muon DPG-PH Meeting on Monday, 2 April 2012. Link to agenda: https://indico.cern.ch/conferenceDisplay.py?confId=184519
} 

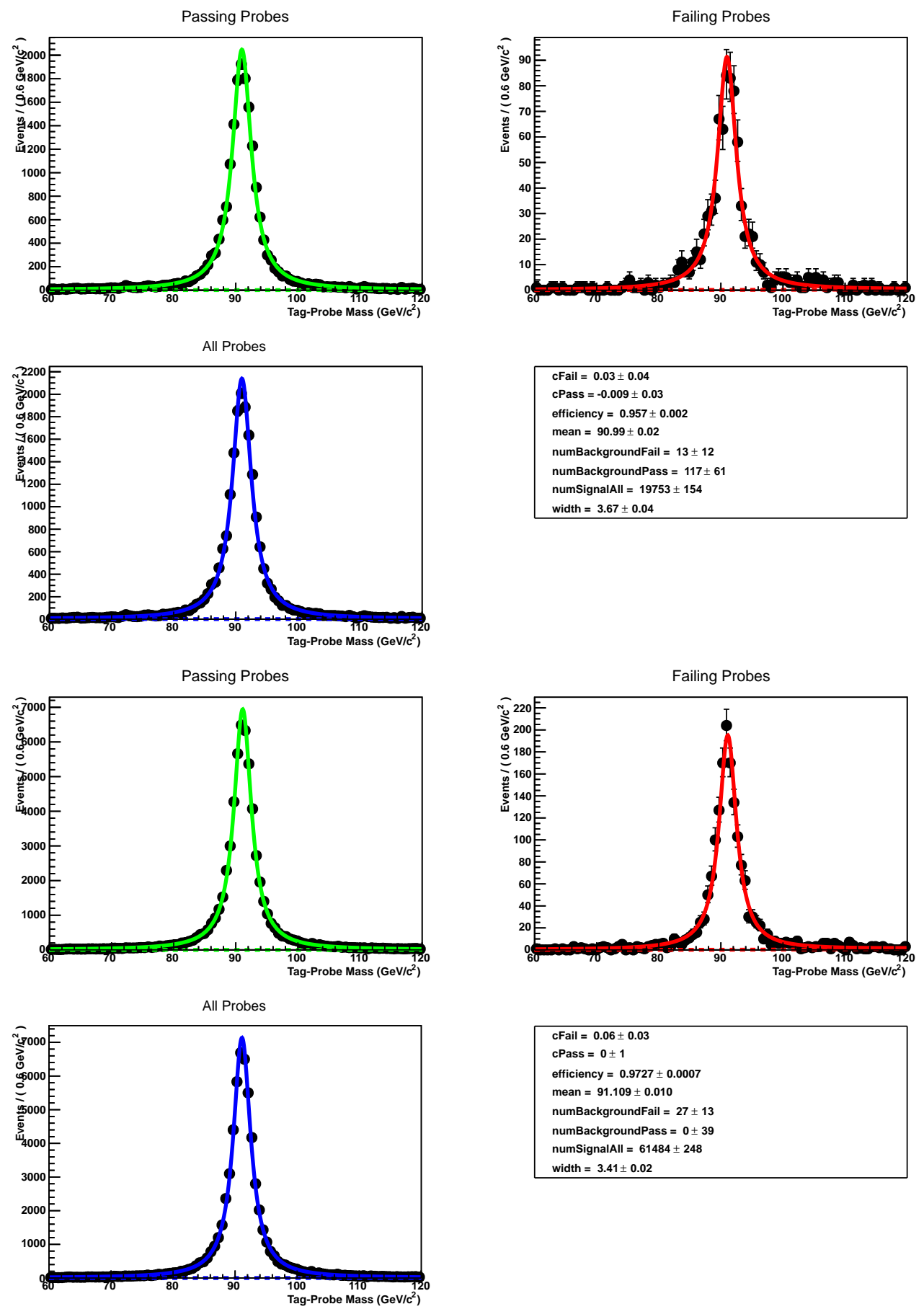

Figure 5.22: Distributions of dimuon masses consistent with $Z$ mass (from 60 to $120 \mathrm{GeV}$ ) containing passing, failing, and all probe muons in tag and probe study of HLT_Mu17_Mu8 trigger efficiency for high- $p_{T}$ probe muons with $p_{T}(\mu)>17 \mathrm{GeV}$ and $|\eta|<0.9$. Top four: data; bottom four: MC. 

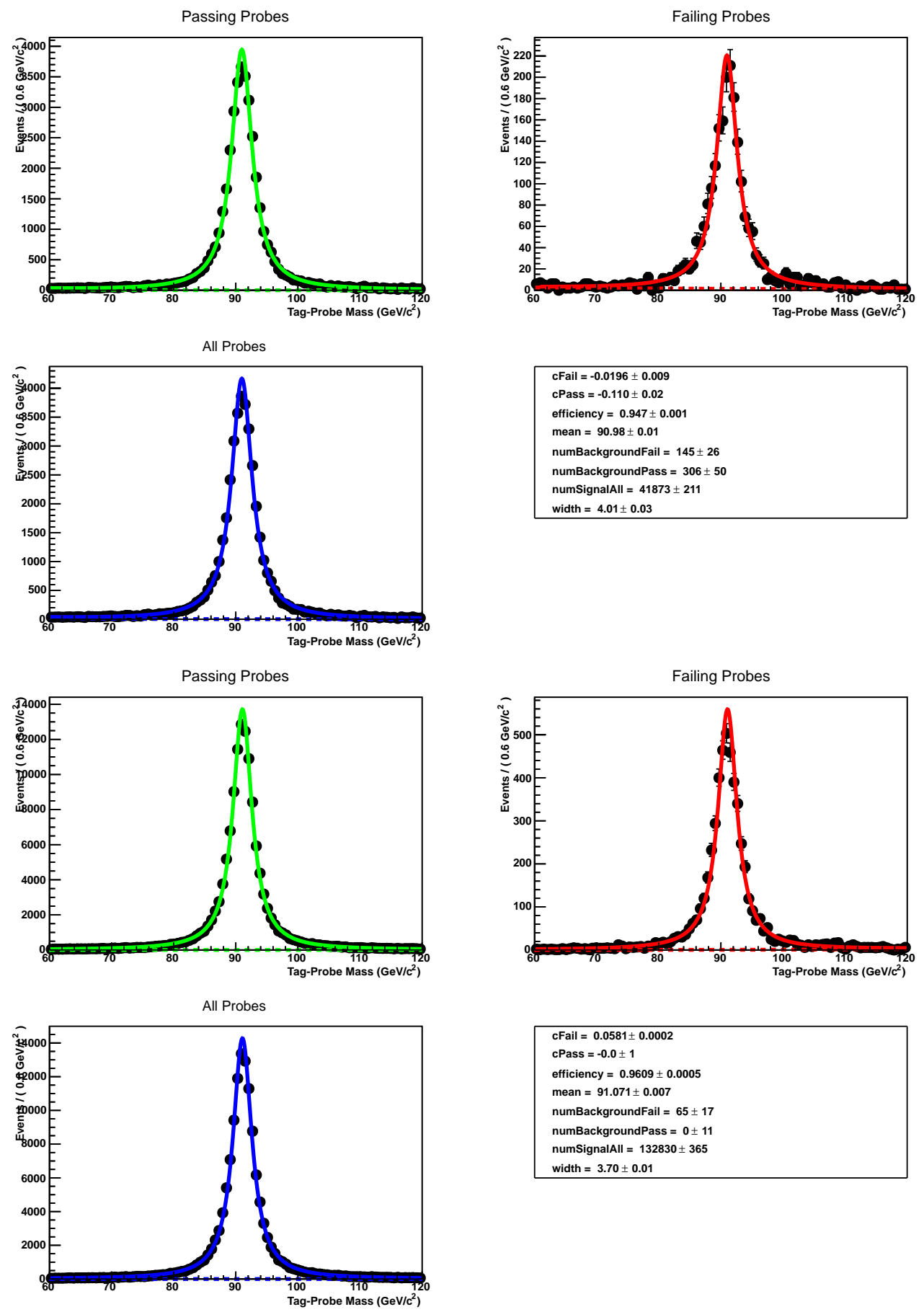

Figure 5.23: Distributions of dimuon masses consistent with $Z$ mass (from 60 to $120 \mathrm{GeV}$ ) containing passing, failing, and all probe muons in tag and probe study of HLT_Mu17_Mu8 trigger efficiency f or low- $p_{T}$ probe muons with $p_{T}(\mu)>8 \mathrm{GeV}$ and $|\eta|<2.4$. Top four: data; bottom four: MC. 
ciencies. We need to find how the per event efficiency would change in simulation if the per leg efficiencies are to be lower (the scale factor is $f_{\text {leg }}=\epsilon_{H L T}^{\text {data }} / \epsilon_{H L T}^{s i m}=0.984$ ). We choose a conservative approach in which we vary the degree of correlation of reconstructing muons in the trigger when there are two true muons present between zero and one, which is conservative as this approach removes reliance of the simulation to properly reproduce these correlations. In the case of no correlations, the efficiency would be $\epsilon_{\text {evt }}^{\text {cor }=0 \%}=1-\left(1-\epsilon_{\text {leg }}\right)^{4}-4 \cdot \epsilon_{l e g} \cdot\left(1-\epsilon_{l e g}\right)^{3}$, while if the probability of finding at least one muon of the two is determined by the probability to find the "better" muon (the $100 \%$ correlated case) $\epsilon_{\text {evt }}^{\text {cor }=100 \%}=1-\left(1-\epsilon_{l e g}\right)^{2}-2 \cdot \epsilon_{l e g} \cdot\left(1-\epsilon_{l e g}\right)$.

Table 5.16 shows the result of the calculations of $\epsilon_{\text {evt }}^{c o r=0 \%}$ and $\epsilon_{\text {evt }}^{c o r=100 \%}$ taking the per leg efficiency to be either $\epsilon_{l e g}=96.7 \%$ (simulation) or $\epsilon_{l e g}=95.2 \%$ (data). The ratio $R$ of the per event efficiencies for each of the two cases (the correlated and uncorrelated) shows the sensitivity of the per-event efficiency to variations of the the per-leg efficiency in each limiting case, while the true sensitivity should be somewhere in between. We therefore define the final scale factor for per event efficiency as $f_{H L T}^{e v t}=\left(R^{c o r=0 \%}+R^{c o r=100 \%}\right) / 2=0.984$ and take the uncertainty as $\Delta f_{H L T}^{e v t}=\left|R^{c o r=0 \%}-R^{c o r=100 \%}\right| / 2=0.015$. This scale factor is used to correct simulation prediction for trigger efficiency and the uncertainty is used as the corresponding systematic uncertainty for trigger efficiency. We ignore statistical uncertainties in the scale factors as they are much smaller than the resulting systematic uncertainty.

\subsubsection{Muon Isolation Scale Factor}

In the study of the efficiency of dimuon isolation requirement an isolated muon $\left(I_{\text {trk }}^{a b s}(\mu)<3 \mathrm{GeV}\right)$ with transverse momentum $p_{T}>40 \mathrm{GeV}$ that passed all muon identification quality selections used in the analysis and matched to a trigger object in HLT_Mu40* and HLT_Mu17_Mu8* paths has been defined as a tag. A probe is 


\begin{tabular}{|c|c|c|c|}
\hline & Data & Simulation & Data/Simulation Ratio \\
\hline$\epsilon_{\text {leg }}$ & $0.952 \pm 0.002$ & $0.967 \pm 0.001$ & $0.984 \pm 0.002$ \\
$\epsilon_{\text {evt }}^{\text {cov }=0 \%}$ & 1.000 & 1.000 & 1.000 \\
$\epsilon_{\text {evt }}^{\text {cor }=100 \%}$ & $0.906 \pm 0.004$ & $0.935 \pm 0.002$ & $0.969 \pm 0.004$ \\
\hline
\end{tabular}

Table 5.16: Illustration of the derivation of the scale factor for per-event efficiency using measured per leg efficiencies in data and simulation. The per event scale factor is taken as the average of the Data/Simulation ratios for correlated and uncorrelated cases $\left(f_{H L T}^{e v t}=\left(R^{\text {cor }=0 \%}+R^{\text {cor }=100 \%}\right) / 2=0.984\right)$ and the uncertainty is taken to cover the difference between the two $\left(\Delta f_{H L T}^{e v t}=\left|R^{\text {cor }=0 \%}-R^{\text {cor }=100 \%}\right| / 2=0.015\right)$.

a muon that passed all muon identification quality selections used in the analysis and matched to a trigger object in HLT_Mu17_Mu8* path. If the probe is isolated $\left(I_{t r k}^{a b s}(\mu)<3 \mathrm{GeV}\right)$, then it is defined as passing probe. Note, that usage of the $Z \rightarrow \mu \mu$ samples (data and MC) in this method effectively leads to proper inclusion of kinematic characteristics of signal events as $Z$ and Higgs boson production final states are similar, with the efficiencies dominated by the underlying event and only occasional initial state radiation jets, thus reducing potential systematic effects. Standard output of the T\&P application is shown in Fig. 5.24. The resulting scale factor between data and MC is set to

$$
f_{\text {Iso }}=0.988 \pm 0.001(\text { stat })
$$




\subsection{Findings and Statistical Interpretation}

\subsubsection{Model-Independent Limits}

When the data satisfying all analysis selections have been unblinded, no events have been observed in the signal region, as illustrated in Fig. 5.25 (left). Three events in the off-diagonal region have been observed (see Fig. 5.25 (left) and Table 5.17), which allows a simple estimate of the expected rate of $(3 \pm \sqrt{3}) \times 0.18 / 0.82=0.7 \pm 0.4$ $b \bar{b}$ background events in the diagonal signal region. After combination with $0.3 \pm 0.3$ direct $J / \psi J / \psi$ background events the expected number of background events in the signal region is $1.0 \pm 0.5$, which is consistent with zero events observed in the signal


Figure 5.25: The distribution $m_{1}$ versus $m_{2}$ of masses of the dimuons for data events (shown as empty circles) surviving all selections except the requirement that these two masses fall into the diagonal signal region $m_{1} \approx m_{2}$ (outlined with dashed lines). The background expectation (shown in color) is a sum of the $b \bar{b}(2 \mathrm{D}$ template normalized to number of events in the off-diagonal region) and the direct $J / \psi \mathrm{J} / \psi$ production contributions. Left: the analysis tight isolation requirement for dimuons

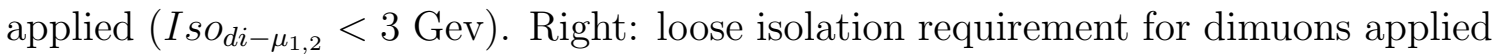
$\left(3<\right.$ sso $\left._{d i-\mu_{1,2}}<8 \mathrm{Gev}\right)$. 
region.

Following the Bayesian prescription [49], the observation of zero events in the signal region can be presented as a 95\% credibility level (CL) one-sided upper limit on the number of events passing full analysis selection:

$$
\begin{gathered}
\sigma(p p \rightarrow 2 a+X) \times \mathcal{L} \times \mathcal{B}^{2}(a \rightarrow 2 \mu) \times \epsilon_{\text {full }}<3 \text { events } \\
\sigma(p p \rightarrow 2 a+X) \times \mathcal{B}^{2}(a \rightarrow 2 \mu) \times \epsilon_{\text {full }}<0.57 \mathrm{fb}
\end{gathered}
$$

where $\mathcal{L}=5.3 \mathrm{fb}^{-1}$ is the total integrated luminosity of the dataset used in this analysis and $\epsilon_{\text {full }}$ is the efficiency of full analysis selection. After incorporating the total systematic uncertainty of $8.0 \%$ (Sec. 5.5) into the upper limit as prescribed in [149] and using the weak model-dependence of the ratio $r=\epsilon_{\text {full }} / \alpha_{\text {gen }}=0.67 \pm 0.06$ (Sec. 5.3), one can rewrite the upper limit above as:

$$
\sigma(p p \rightarrow 2 a+X) \times \mathcal{B}^{2}(a \rightarrow 2 \mu) \times \alpha_{\text {gen }}<0.86 \mathrm{fb},
$$

where $\bar{r}$ is the central value of $r$, and $\alpha_{\text {gen }}$ is the generator-level kinematic and geometric acceptance.

The limit presented in this form allows a simple reinterpretation of the results

\begin{tabular}{|c|c|c|c|c|c|}
\hline Run \# & Event \# & $\begin{array}{c}\text { mass of the triggered } \\
\text { dimuon }(\mathrm{GeV})\end{array}$ & $\begin{array}{c}\text { mass of the other } \\
\text { dimuon }(\mathrm{GeV})\end{array}$ & $\begin{array}{c}\text { isolation of the triggered } \\
\text { dimuon }(\mathrm{GeV})\end{array}$ & $\begin{array}{c}\text { isolation of the other } \\
\text { dimuon }(\mathrm{GeV})\end{array}$ \\
\hline 167674 & 302005714 & 2.10 & 0.50 & 0.00 & 0.00 \\
172033 & 757640577 & 1.95 & 3.12 & 0.00 & 0.00 \\
172635 & 382480189 & 0.49 & 0.78 & 0.51 & 2.20 \\
\hline
\end{tabular}

Table 5.17: List of data events with two dimuons (and any number of orphan muons) surviving all analysis selections, but applying the requirement that the two masses fall into the off-diagonal region with the analysis tight dimuon isolation requirement $\left(\right.$ Iso $\left._{d i-\mu_{1,2}}<3 \mathrm{GeV}\right)$. 
in other models. This requires calculation of $\alpha_{\text {gen }}$ using generator-level information. The limit is applicable to models with two pairs of muons coming from light bosons of the same type with a mass in range $0.25<m_{a}<3.55 \mathrm{GeV}$, where the new light bosons are typically isolated, spatially separated to not be vetoed by the isolation requirement and have no substantial lifetime. The efficiency of the selections in this analysis abruptly deteriorates if the light boson's decay vertex is more than $\sim 4 \mathrm{~cm}$ from the beamline in the transverse plane (see Sec. 5.6.3).

\subsubsection{Limits in Benchmark Scenarios}

We interpret these results in the context of the NMSSM and the dark-SUSY benchmark models, taking into account the dependence of the signal selection efficiencies on $m_{h}$ and $m_{a}$, and derive 95\% CL upper limits on the product of the cross section and branching fraction, using a Bayesian prescription. We also compare the derived experimental limits with a few simplified new physics scenarios. In the representative models, for any fixed combinations of $m_{h}$ and $m_{a}$ both the Higgs boson production cross section and the branching fractions can vary significantly, depending on the choice of parameters. In the absence of broadly accepted benchmark scenarios, we normalize the production cross sections in these examples to that of the SM Higgs boson [148].

\begin{tabular}{|c|c|c|c|c|c|}
\hline Run \# & Event \# & $\begin{array}{c}\text { mass of the triggered } \\
\text { dimuon }(\mathrm{GeV})\end{array}$ & $\begin{array}{c}\text { mass of the other } \\
\text { dimuon }(\mathrm{GeV})\end{array}$ & $\begin{array}{c}\text { isolation of the triggered } \\
\text { dimuon }(\mathrm{GeV})\end{array}$ & $\begin{array}{c}\text { isolation of the other } \\
\text { dimuon }(\mathrm{GeV})\end{array}$ \\
\hline 172868 & 910039051 & 0.86 & 1.77 & 6.21 & 5.52 \\
177141 & 337540844 & 3.06 & 1.73 & 5.12 & 5.77 \\
178421 & 835925618 & 1.75 & 0.55 & 5.58 & 4.72 \\
180076 & 459633211 & 2.23 & 1.73 & 3.91 & 6.77 \\
\hline
\end{tabular}

Table 5.18: List of data events with two dimuons (and any number of orphan muons) surviving all analysis selections, but applying the requirement that the two masses fall into the off-diagonal region and the analysis loose dimuon isolation requirement for $\left(3<I s o_{d i-\mu_{1,2}}<8 \mathrm{GeV}\right)$ 

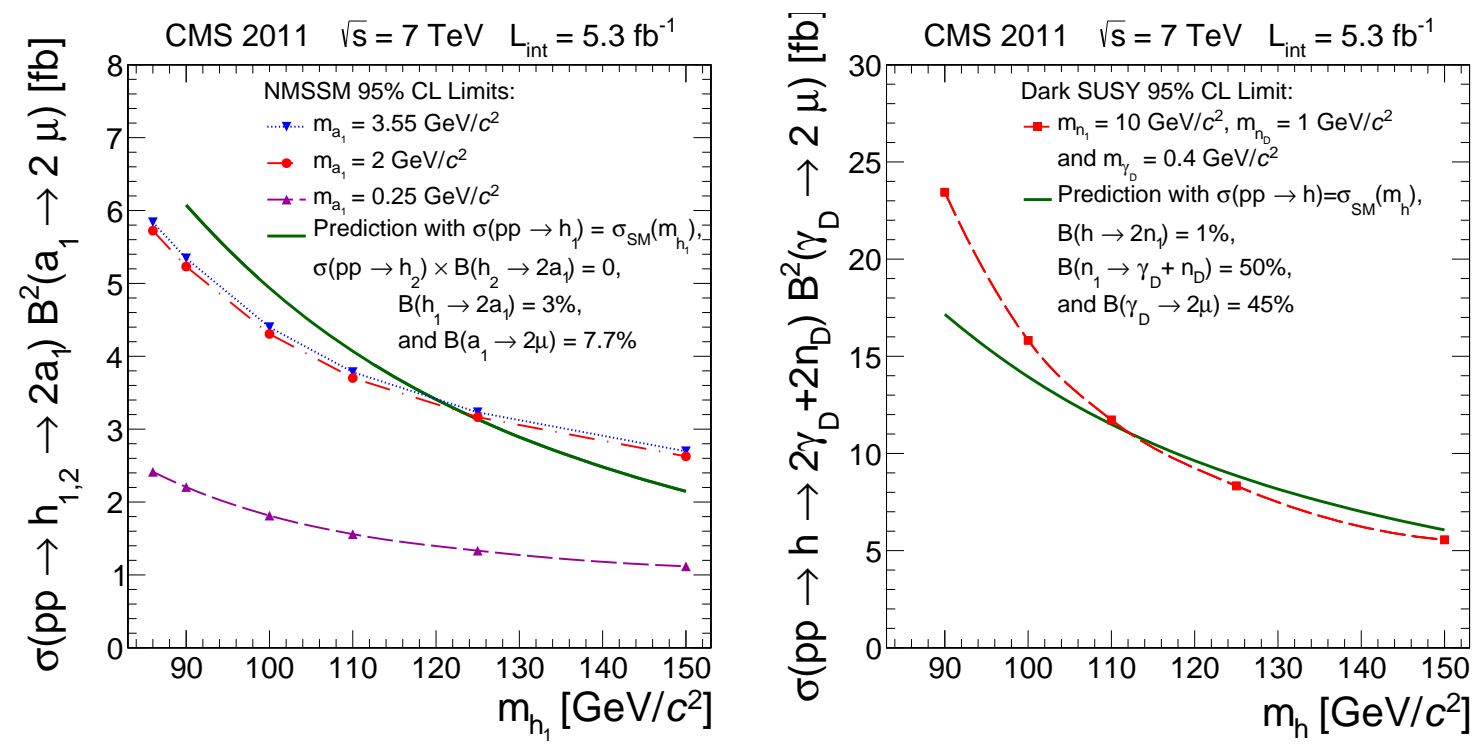

Figure 5.26: Left: The 95\% CL upper limits as functions of $m_{h_{1}}$, for the NMSSM case, on $\sigma\left(p p \rightarrow h_{1,2} \rightarrow 2 a_{1}\right) \times \mathcal{B}^{2}\left(a_{1} \rightarrow 2 \mu\right)$ with $m_{a_{1}}=0.25 \mathrm{GeV}$ (dashed curve), $m_{a_{1}}=2$ $\mathrm{GeV}$ (dash-dotted curve) and $m_{a_{1}}=3.55 \mathrm{GeV}$ (dotted curve). As an illustration, the limits are compared to the predicted rate (solid curve) obtained using a simplified scenario with $\sigma\left(p p \rightarrow h_{1}\right)=\sigma_{\mathrm{SM}}\left(m_{h_{1}}\right)$ [148], $\sigma\left(p p \rightarrow h_{2}\right) \times \mathcal{B}\left(h_{2} \rightarrow 2 a_{1}\right)=0$, $\mathcal{B}\left(h_{1} \rightarrow 2 a_{1}\right)=3 \%$, and $\mathcal{B}\left(a_{1} \rightarrow 2 \mu\right)=7.7 \%$. The chosen $\mathcal{B}\left(a_{1} \rightarrow 2 \mu\right)$ is taken from [108] for $m_{a_{1}}=2 \mathrm{GeV}$ and NMSSM parameter $\tan \beta=20$. Right: The $95 \%$ CL upper limit as a function of $m_{h}$, for the dark-SUSY case, on $\sigma\left(p p \rightarrow h \rightarrow 2 n_{1} \rightarrow\right.$ $\left.2 n_{D}+2 \gamma_{D}\right) \times \mathcal{B}^{2}\left(\gamma_{D} \rightarrow 2 \mu\right)$ with $m_{n_{1}}=10 \mathrm{GeV}, m_{n_{D}}=1 \mathrm{GeV}$ and $m_{\gamma_{D}}=0.4 \mathrm{GeV}$ (dashed curve). As an illustration, the limit is compared to the predicted rate (solid curve) obtained using a simplified scenario with SM Higgs boson production cross section $\sigma(p p \rightarrow h)=\sigma_{\mathrm{SM}}\left(m_{h}\right)[148], \mathcal{B}\left(h \rightarrow 2 n_{1}\right)=1 \%, \mathcal{B}\left(n_{1} \rightarrow n_{D}+\gamma_{D}\right)=50 \%$, and $\mathcal{B}\left(\gamma_{D} \rightarrow 2 \mu\right)=45 \%$. The chosen $\mathcal{B}\left(\gamma_{D} \rightarrow 2 \mu\right)$ is taken from [150] for $m_{\gamma_{D}}=0.4$ $\mathrm{GeV}$. 

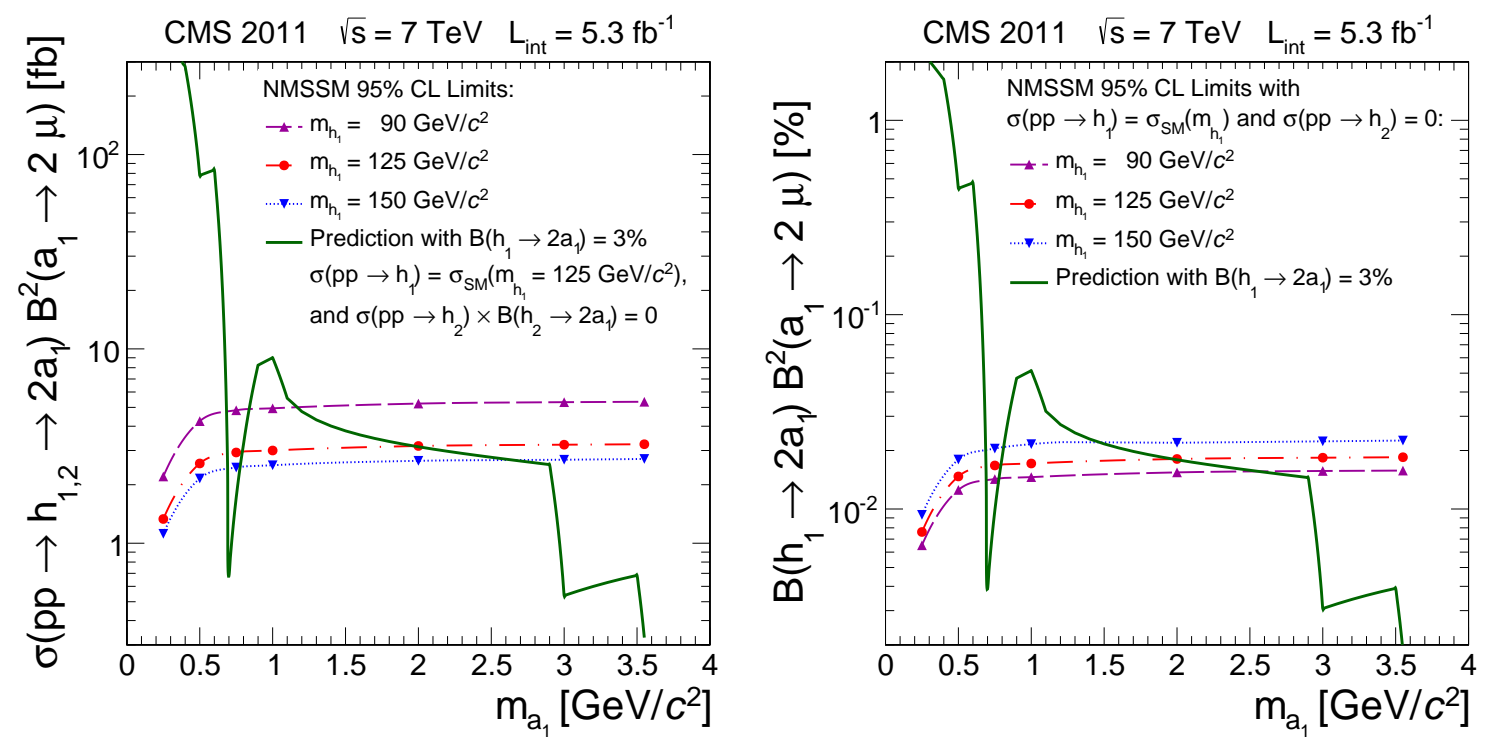

Figure 5.27: Left: The 95\% CL upper limits as functions of $m_{a_{1}}$, for the NMSSM case, on $\sigma\left(p p \rightarrow h_{1,2} \rightarrow 2 a_{1}\right) \times \mathcal{B}^{2}\left(a_{1} \rightarrow 2 \mu\right)$ with $m_{h_{1}}=90 \mathrm{GeV}$ (dashed curve), $m_{h_{1}}=125 \mathrm{GeV}$ (dash-dotted curve) and $m_{h_{1}}=150 \mathrm{GeV}$ (dotted curve). The limits are compared to the predicted rate (solid curve) obtained using a simplified scenario with $\mathcal{B}\left(h_{1} \rightarrow 2 a_{1}\right)=3 \%, \sigma\left(p p \rightarrow h_{1}\right)=\sigma_{\mathrm{SM}}\left(m_{h_{1}}=125\right) \mathrm{GeV}$ [148], $\sigma\left(p p \rightarrow h_{2}\right) \times \mathcal{B}\left(h_{2} \rightarrow 2 a_{1}\right)=0$, and $\mathcal{B}\left(a_{1} \rightarrow 2 \mu\right)$ as a function of $m_{a_{1}}$ which is taken from [108] for NMSSM parameter $\tan \beta=20$. Right: The 95\% CL upper limits on $\mathcal{B}\left(h_{1} \rightarrow 2 a_{1}\right) \times \mathcal{B}^{2}\left(a_{1} \rightarrow 2 \mu\right)$ with $m_{h_{1}}=90 \mathrm{GeV}$ (dashed curve), $m_{h_{1}}=125 \mathrm{GeV}$ (dash-dotted curve) and $m_{h_{1}}=150 \mathrm{GeV}$ (dotted curve) assuming $\sigma\left(p p \rightarrow h_{1}\right)=$ $\sigma_{\mathrm{SM}}\left(m_{h_{1}}\right)[148]$ and $\sigma\left(p p \rightarrow h_{2}\right) \times \mathcal{B}\left(h_{2} \rightarrow 2 a_{1}\right)=0$. The limits are compared to the predicted branching fraction (solid line) obtained using a simplified scenario with $\mathcal{B}\left(h_{1} \rightarrow 2 a_{1}\right)=3 \%$ and $\mathcal{B}\left(a_{1} \rightarrow 2 \mu\right)$ as a function of $m_{a_{1}}$ which is taken from [108] for NMSSM parameter $\tan \beta=20$. 
For the NMSSM, the 95\% CL upper limit is derived for $\sigma\left(p p \rightarrow h_{1,2} \rightarrow 2 a_{1}\right) \times$ $\mathcal{B}^{2}\left(a_{1} \rightarrow 2 \mu\right)$ as a function of $m_{h_{1}}$ for three choices of $m_{a_{1}}$ as shown in Fig. 5.26 (left) and as a function of $m_{a_{1}}$ for three choices of $m_{h_{1}}$ as shown in Fig. 5.27 (left). As $m_{h_{2}}$ is unrestricted for any given $m_{h_{1}}$, we use $\epsilon_{\text {full }}\left(m_{h_{2}}\right)=\epsilon_{\text {full }}\left(m_{h_{1}}\right)$ to simplify the interpretation. This is conservative since $\epsilon_{\text {full }}\left(m_{h_{2}}\right)>\epsilon_{\text {full }}\left(m_{h_{1}}\right)$ if $m_{h_{2}}>m_{h_{1}}$, for any $m_{a_{1}}$. We also derive the $95 \%$ CL upper limit for $\mathcal{B}\left(h_{1} \rightarrow 2 a_{1}\right) \times \mathcal{B}^{2}\left(a_{1} \rightarrow 2 \mu\right)$ as a function of $m_{a_{1}}$ for three choices of $m_{h_{1}}$ as shown in Fig. 5.27 (right) assuming that only $h_{1}$ gives a significant contribution to the final state considered in this analysis and has the production cross section of a SM Higgs boson, i.e. $\sigma(p p \rightarrow$ $\left.h_{1}\right)=\sigma_{\mathrm{SM}}\left(m_{h_{1}}\right)$ and $\sigma\left(p p \rightarrow h_{2}\right) \times \mathcal{B}\left(h_{2} \rightarrow 2 a_{1}\right)=0$. For the NMSSM simplified prediction scenario we use $\mathcal{B}\left(a_{1} \rightarrow 2 \mu\right)$ as a function of $m_{a_{1}}$, calculated in [108] for $\tan \beta=20$ with no hadronization effects included in the $m_{a_{1}}<2 m_{\tau}$ region. The branching fraction $\mathcal{B}\left(a_{1} \rightarrow 2 \mu\right)$ is influenced by the $a_{1} \rightarrow s \bar{s}$ and $a_{1} \rightarrow g g$ channels. Noticeable structures in the predicted curves visible in Fig. 5.27 arise from the fact that $\mathcal{B}\left(a_{1} \rightarrow g g\right)$ varies rapidly in that region of $m_{a_{1}}$. The rapid variation in $\mathcal{B}\left(a_{1} \rightarrow g g\right)$ occurs when $m_{a_{1}}$ crosses the internal quark loop thresholds. The representative value of $\mathcal{B}\left(a_{1} \rightarrow 2 \mu\right)$ is equal to $7.7 \%$ for $m_{a_{1}} \approx 2 \mathrm{GeV}$. Finally, to simplify visual comparisons, we choose $\mathcal{B}\left(h_{1} \rightarrow 2 a_{1}\right)=3 \%$, which yields predictions for the rates of dimuon pair events comparable to the obtained experimental limits.

In the case of the dark-SUSY model, the 95\% CL upper limit is derived for $\sigma\left(p p \rightarrow h \rightarrow 2 n_{1} \rightarrow 2 n_{D}+2 \gamma_{D}\right) \times \mathcal{B}^{2}\left(\gamma_{D} \rightarrow 2 \mu\right)$ as a function of $m_{h}$. This limit is shown in Fig. 5.26 (right) for $m_{n_{1}}=10 \mathrm{GeV}, m_{n_{D}}=1 \mathrm{GeV}$ and $m_{\gamma_{D}}=0.4 \mathrm{GeV}$. For the dark-SUSY simplified scenario we use the branching fraction $\mathcal{B}\left(\gamma_{D} \rightarrow 2 \mu\right)$ close to its maximum at $m_{\gamma_{D}}=0.4 \mathrm{GeV}$, of $45 \%$, calculated in [150]. We also use $\mathcal{B}\left(n_{1} \rightarrow n_{D}+\gamma_{D}\right)=50 \%$, allowing for other possible decays. Finally, to simplify visual comparisons, we choose $\mathcal{B}\left(h \rightarrow 2 n_{1}\right)=1 \%$, which yields predictions for the 


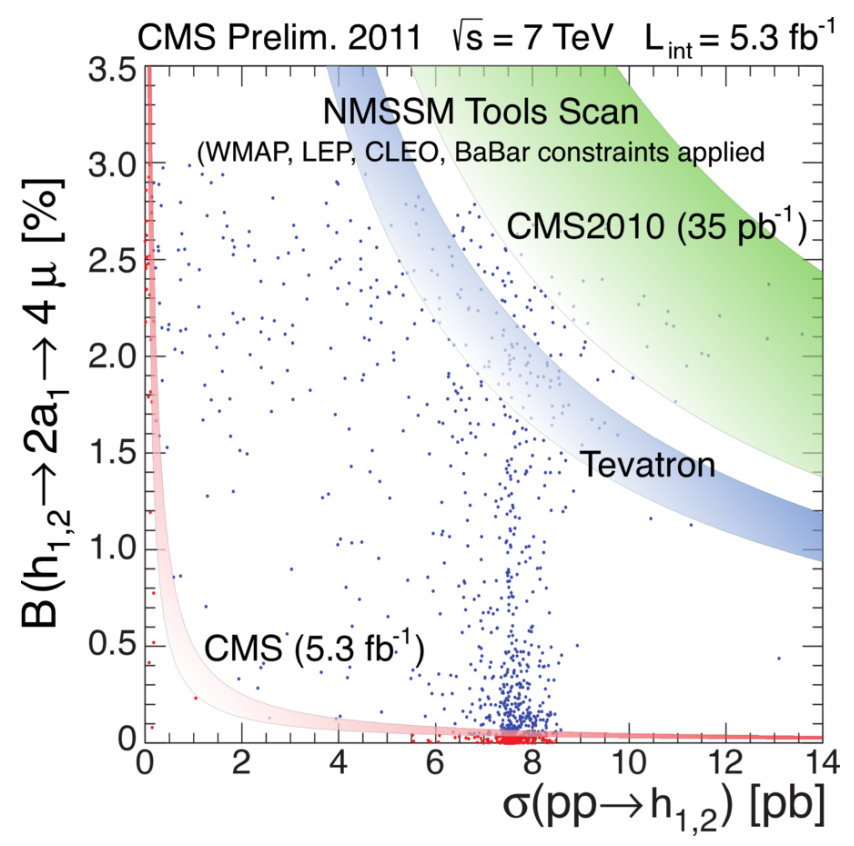

Figure 5.28: The 95\% C.L. upper limit on the allowed NMSSM models consistent with LEP, WMAP, CLEO/BaBar measurements in the plane of $B\left(h_{1,2} \rightarrow a_{1} a_{1} \rightarrow 4 \mu\right)$ versus $\sigma\left(p p \rightarrow h_{1,2}\right)$ for $\sqrt{s}=7 \mathrm{TeV}$. Exact limits depend on the acceptance for each model point and therefore do not appear as strict curves. The area bounded between solid curves restricts the range of limits that correspond to the range of acceptances for allowed models. For comparison the result of the previous CMS search [140, 152] (the range of upper limits delineated by the magenta dashed curves) and similar Tevatron search [151] (the range of upper limits delineated by the green dash-dotted lines) are shown.

rates of dimuon pair events comparable to the obtained experimental limits.

To evaluate the power of the obtained limits in the context of NMSSM, we scan the NMMSM parameter space using the NMSSM Tools package, select model points with $2 m_{\mu}<m_{a_{1}}<2 m_{\tau}$ consistent with the experimental data from LEP, WMAP, and CLEO/BaBar measurements. The density of NMSSM points is plotted in the plane of production cross section $\sigma\left(p p \rightarrow h_{1,2}\right)$ and decay branching fraction to muons $B\left(h_{1,2} \rightarrow 2 a_{1} \rightarrow 4 \mu\right)$ in Fig. 5.28. Note that for each NMSSM point the cross section and the branching fraction can be calculated for both CP-even Higgs bosons, $h_{1}$ 

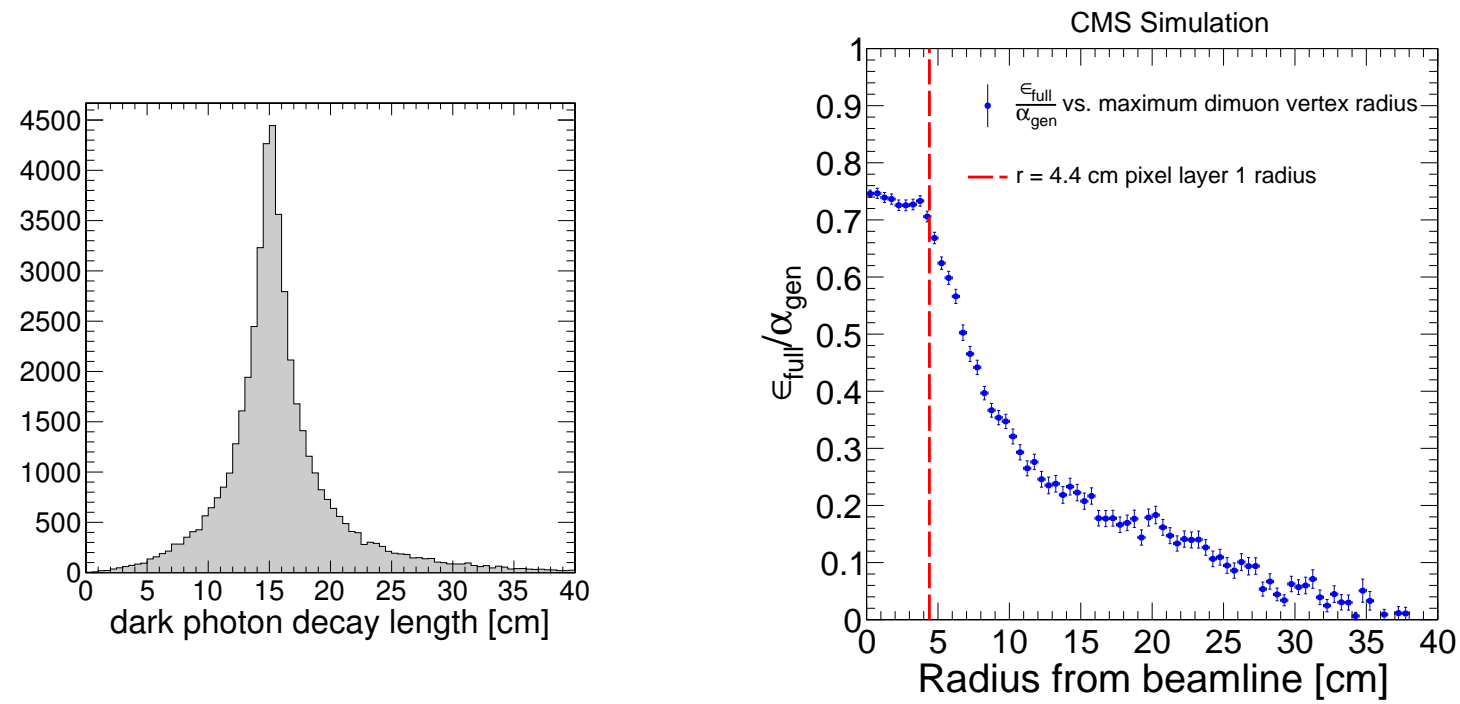

Figure 5.29: Left: distribution of $c \tau$, the real decay length of dark photons in signal sample with the nominal decay length $c \tau$ is set to $15 \mathrm{~cm}$. Right: $\alpha_{\text {reco }} / \alpha_{\text {gen }}$ dependence on the maximum dimuon displaced vertex radius.

and $h_{2}$. The production rate of the pair of CP-odd Higgs bosons $a_{1}$ depends on the singlet fraction of CP-even Higgs bosons $h_{1}$ and $h_{2}$. Both production rates have been calculated, but only the largest value of these two rates is plotted. Obtained in this analysis experimental exclusion limit is confined between solid lines because for a particular model point rate limit depends on the acceptance. As a result, the density distributions of NMSSM points clearly demonstrate that this analysis significantly restricts the allowed parameter space of the NMSSM and surpasses the limits measured at Tevatron [151] and LHC with data collected in $2010[140,152]$.

\subsubsection{Future Interpretations}

Depending on the model of interest, new light bosons may be substantially longlived to lead to the displaced dimuon vertices fall inside the pixel or silicon tracker. We study the sensitivity of the analysis to such displacement measuring the ratio 
of $\alpha_{\text {reco }}$, efficiency of all analysis selections on reconstruction level, and $\alpha_{\text {gen }}$, basic kinematic and geometrical acceptance on generator level, using simulation of dark SUSY benchmark model with varying dark photon nominal decay length. For each particle, a nominal decay length value, $c \tau$, is used to calculate a real decay length $c \tau^{\text {real }}=-c \tau \cdot \log (\alpha)$, where $\alpha$ is a random number from 0 to 1 . As a result, the real decay length is smeared around the nominal value: Fig. 5.29(left) shows one example for a sample where $c \tau=15 \mathrm{~cm}$. Table 5.19 summarizes the $\alpha_{\text {reco }} / \alpha_{\text {gen }}$ ratios depending on the nominal decay length $c \tau$ :

\begin{tabular}{|c|c|c|c|}
\hline$\gamma_{\text {dark }}$ decay length, $\mathrm{cm}$ & $\alpha_{\text {reco }} / \alpha_{\text {gen }}, \%$ & $\gamma_{\text {dark }}$ decay length, $\mathrm{cm}$ & $\alpha_{\text {reco }} / \alpha_{\text {gen }}, \%$ \\
\hline \hline 0.1 & $74.6 \pm 0.6$ & 9 & $34.9 \pm 0.7$ \\
1 & $74.7 \pm 0.6$ & 10 & $33.1 \pm 0.7$ \\
2 & $73.6 \pm 0.6$ & 12 & $26.3 \pm 0.7$ \\
3 & $72.1 \pm 0.7$ & 15 & $20.3 \pm 0.6$ \\
4 & $69.2 \pm 0.7$ & 18 & $16.6 \pm 0.6$ \\
5 & $63.5 \pm 0.7$ & 21 & $13.8 \pm 0.5$ \\
6 & $56.2 \pm 0.8$ & 24 & $10.7 \pm 0.4$ \\
8 & $42.5 \pm 0.7$ & 30 & $5.8 \pm 0.3$ \\
\hline
\end{tabular}

Table 5.19: Dependence of $\alpha_{\text {reco }} / \alpha_{\text {gen }}$ on $c \tau$, the nominal decay length of dark photons.

The source of the efficiency loss may be more evident on the plot of $\alpha_{\text {reco }} / \alpha_{\text {gen }}$ dependence on dimuon displaced vertex radius with respect to the beamline. Since two dark photons with the same nominal decay length may produce vertices with substantially different radii, we plot the ratio versus the maximum radius out of two as it dominates the efficiency loss (see Fig. 5.29(right)). The ratio deteriorates if new bosons' lifetime is sufficiently large for them to reach the first layer of the pixel tracker $(\sim 4-5 \mathrm{~cm})$. Therefore, the earlier obtained model-independent limit is not applicable to models where the new light bosons have lifetimes corresponding 
to the flight distances over a few $\mathrm{cm}$ from the beamline before their decay. One can still obtain a conservative limit for such cases by modifying $\alpha_{\text {gen }}$ to include the requirement that the new bosons decay before $r=4 \mathrm{~cm}$ from the beamline. 


\section{CONCLUSIONS *}

A search for non-standard-model Higgs boson decays to pairs of new light bosons, which subsequently decay to pairs of oppositely charged muons $(h \rightarrow 2 a+X \rightarrow$ $4 \mu+X)$ has been presented. The search is based on a data sample corresponding to an integrated luminosity of $5.3 \mathrm{fb}^{-1}$ collected by the CMS experiment in protonproton collisions at $\sqrt{s}=7 \mathrm{TeV}$ in 2011. No excess is observed with respect to the SM predictions. An upper limit at $95 \%$ confidence level on the product of the cross section times branching fraction times acceptance is obtained. The limit is valid for new light-boson masses in the range $0.25<m_{a}<3.55 \mathrm{GeV}$ and for Higgs boson masses in the range $m_{h}>86 \mathrm{GeV}$. The results have been interpreted in the context of the NMSSM and the dark-SUSY benchmark models. The analysis has been designed as a quasi-model-independent search allowing interpretation of its results in the context of a broad range of new physics scenarios predicting the same type of signature. In the context of the NMSSM and one of the SUSY models with hidden valleys this search provides the best experimental limits to date, significantly surpassing the sensitivity of similar searches performed at the Tevatron.

\footnotetext{
* Reprinted with permission from "Search for a Non-Standard-Model Higgs Boson Decaying to a Pair of New Light Bosons in Four-Muon Final States" by S. Chatrchyan et al., 2013. Physics Letters B $\mathbf{7 2 6}$ 564-586, Copyright 2013 by CERN
} 


\section{REFERENCES}

[1] J. J. Thomson M.A.F.R.S. "XL. Cathode Rays." Philosophical Magazine Series 544 (1897) 293-316.

[2] E. D. Bloom et al. "High-Energy Inelastic e-p Scattering at $6^{\circ}$ and $10^{\circ}$." Phys. Rev. Lett. 23 (1969) 930-934.

[3] M. Breidenbach et al. "Observed Behavior of Highly Inelastic Electron-Proton Scattering." Phys. Rev. Lett. 23 (1969) 935-939.

[4] J. E. Augustin et al. "Discovery of a Narrow Resonance in $e^{+} e^{-}$Annihilation." Phys. Rev. Lett. 33 (1974) 1406-1408.

[5] J. J. Aubert et al. "Experimental Observation of a Heavy Particle J." Phys. Rev. Lett. 33 (1974) 1404-1406.

[6] S. W. Herb et al. "Observation of a Dimuon Resonance at $9.5 \mathrm{GeV}$ in $400-\mathrm{GeV}$ Proton-Nucleus Collisions." Phys. Rev. Lett. 39 (1977) 252-255.

[7] F. Abe et al. "Observation of Top Quark Production in $\bar{p} p$ Collisions with the Collider Detector at Fermilab." Phys. Rev. Lett. 74 (1995) 2626-2631.

[8] R. Brandelik et al. "Evidence for Planar Events in $e^{+} e^{-}$Annihilation at HighEnergies." Phys. Lett. B 86 (1979) 243.

[9] D. P. Barber et al. "Discovery of Three Jet Events and a Test of Quantum Chromodynamics at PETRA Energies." Phys. Rev. Lett. 43 (1979) 830.

[10] G. Arnison et al. "Experimental Observation of Isolated Large Transverse Energy Electrons with Associated Missing Energy at $\sqrt{s}=540 \mathrm{GeV}$." Phys. Lett. $B 122$ (1983) 103-116. 
[11] M. Banner et al. "Observation of Single Isolated Electrons of High Transverse Momentum in Events with Missing Transverse Energy at the CERN $p \bar{p}$ collider." Phys. Lett. B 122 (1983) 476-485.

[12] G. Arnison et al. "Experimental Observation of Lepton Pairs of Invariant Mass Around $95 \mathrm{GeV} / \mathrm{c}^{2}$ at the CERN SPS collider." Phys. Lett. B 126 (1983) 398410.

[13] P. Bagnaia et al. "Evidence for $Z^{0} \rightarrow e^{+} e^{-}$at the CERN $p \bar{p}$ Collider." Phys. Lett. $B 129$ (1983) 130-140.

[14] S. Chatrchyan et al. "Observation of a New Boson at a Mass of $125 \mathrm{GeV}$ with the CMS Experiment at the LHC." Physics Letters B 716 (2012) 30-61.

[15] G. Aad et al. "Observation of a New Particle in the Search for the Standard Model Higgs Boson with the ATLAS Detector at the LHC." Physics Letters B 716 (2012) 1-29.

[16] C. Burgess and G. Moore. The Standard Model: A Primer. Cambridge University Press, 2012.

[17] P. W. Higgs. "Broken Symmetries and the Masses of Gauge Bosons." Phys. Rev. Lett. 13 (1964) 508-509.

[18] P. W. Higgs. "Spontaneous Symmetry Breakdown without Massless Bosons." Phys. Rev. 145 (1966) 1156-1163.

[19] F. Englert and R. Brout. "Broken Symmetry and the Mass of Gauge Vector Mesons." Phys. Rev. Lett. 13 (1964) 321-323.

[20] G. S. Guralnik, C. R. Hagen, and T. W. B. Kibble. "Global Conservation Laws and Massless Particles." Phys. Rev. Lett. 13 (1964) 585-587. 
[21] T. W. B. Kibble. "Symmetry Breaking in Non-Abelian Gauge Theories." Phys. Rev. 155 (1967) 1554-1561.

[22] S. Chatrchyan et al. "The CMS xEperiment at the CERN LHC." Journal of Instrumentation 3 (2008) S08004.

[23] G. Aad et al. "The ATLAS Experiment at the CERN Large Hadron Collider." Journal of Instrumentation 3 (2008) S08003.

[24] A. Breskin and R. Voss. The CERN Large Hadron Collider: Accelerator and Experiments. CERN, Geneva, 2009.

[25] S. Glashow. "Partial Symmetries of Weak Interactions." Nucl.Phys. 22 (1961) $579-588$.

[26] A. Salam and N. Svartholm. "Elementary Particle Theory." Almquist and Wiksell, Stockholm (1968) 367.

[27] S. Weinberg. "A Model of Leptons." Phys. Rev. Lett. 19 (1967) 1264-1266.

[28] Y. Ne'eman. "Derivation of Strong Interactions from a Gauge Invariance." Nuclear Physics 26 (1961) 222-229.

[29] M. Gell-Mann. "Symmetries of Baryons and Mesons." Phys. Rev. 125 (1962) 1067-1084.

[30] M. Gell-Mann. "A Schematic Model of Baryons and Mesons." Physics Letters 8 (1964) 214-215.

[31] G. Zweig. "An $\mathrm{SU}_{3}$ Model for Strong Interaction Symmetry and Its Breaking; Version 2." (1964) 80.

[32] H. Fritzsch and M. Gell-Mann. "Current Algebra: Quarks and What Else?" eConf C720906V2 (1972) 135-165. 
[33] I. Aitchison and A. Hey. Gauge Theories in Particle Physics: A Practical Introduction: From Relativistic Quantum Mechanics to QED, Fourth Edition. v. 2. Taylor \& Francis, 2012.

[34] W. de Boer. "The Discovery of the Higgs Boson with the CMS Detector and its Implications for Supersymmetry and Cosmology." (2013).

[35] D. Ghosh et al. "Looking for an Invisible Higgs Signal at the LHC." Phys. Lett. B725 (2013) 344-351.

[36] LHC Higgs Cross Section Working Group et al. "Handbook of LHC Higgs Cross Sections: 1. Inclusive Observables." CERN-2011-002 (CERN, Geneva, 2011).

[37] LHC Higgs Cross Section Working Group et al. "Handbook of LHC Higgs Cross Sections: 3. Higgs Properties." CERN-2013-004 (CERN, Geneva, 2013).

[38] S. Fukuda et al. "Constraints on Neutrino Oscillations Using 1258 Days of Super-Kamiokande Solar Neutrino Data." Phys. Rev. Lett. 86 (2001) 56565660.

[39] S. Fukuda et al. "Determination of Solar Neutrino Oscillation Parameters Using 1496 Days of Super-Kamiokande-I Data." Physics Letters B 539 (2002) $179-187$.

[40] M. Persic, P. Salucci, and F. Stel. "The Universal Rotation Curve of Spiral Galaxies - I. The Dark Matter Connection." Monthly Notices of the Royal Astronomical Society 281 (1996) 27-47.

[41] D. Clowe et al. "A Direct Empirical Proof of the Existence of Dark Matter." The Astrophysical Journal 648 (2006) 109-113. 
[42] G. Hinshaw et al. "Nine-year Wilkinson Microwave Anisotropy Probe (WMAP) Observations: Cosmological Parameter Results." The Astrophysical Journal Supplement Series 208 (2013) 19.

[43] Planck Collaboration. "Planck 2013 Results. XVI. Cosmological Parameters." $A \& A 571$ (2014) A16.

[44] Y. Nagashima. Beyond the Standard Model of Elementary Particle Physics. Wiley, 2014.

[45] P. Ramond. "Dual Theory for Free Fermions." Phys. Rev. D 3 (1971) 24152418.

[46] J.-L. Gervais and B. Sakita. "Field Theory Interpretation of Supergauges in Dual Models." Nuclear Physics B 34 (1971) 632-639.

[47] J. Iliopoulos and B. Zumino. "Broken Supergauge Symmetry and Renormalization." Nuclear Physics B 76 (1974) 310-332.

[48] H. Goldberg. "Constraint on the Photino Mass from Cosmology." Phys. Rev. Lett. 50 (1983) 1419-1422.

[49] K. A. Olive et al. "Review of Particle Physics." Chin. Phys. C38 (2014) 090001.

[50] N. Arkani-Hamed, S. Dimopoulos, and G. Dvali. "The Hierarchy Problem and New Dimensions at a Millimeter." Physics Letters B 429 (1998) 263-272.

[51] N. Arkani-Hamed, S. Dimopoulos, and G. Dvali. "Phenomenology, Astrophysics, and Cosmology of Theories with Submillimeter Dimensions and $\mathrm{TeV}$ Scale Quantum Gravity." Phys. Rev. D 59 (1999) 086004.

[52] L. Randall and R. Sundrum. "Large Mass Hierarchy from a Small Extra Dimension." Phys. Rev. Lett. 83 (1999) 3370-3373. 
[53] T. Pook. "Extra Dimension-Inspired Models: Z', W', Dijet Resonances, Black Hole Searches." In "12th Conference on the Intersections of Particle and Nuclear Physics (CIPANP 2015) Vail, Colorado, USA, May 19-24, 2015," 2015 $8 a$.

[54] E. J. Eichten, K. D. Lane, and M. E. Peskin. "New Tests for Quark and Lepton Substructure." Phys. Rev. Lett. 50 (1983) 811-814.

[55] M. Tanimoto. "The Effect of the Excited Leptons and the Composite Scalar Bosons on the Processes $e^{+} e^{-} \rightarrow W^{+} W^{-}$or $Z^{0} Z^{0}$." Physics Letters $B \mathbf{1 6 0}$ (1985) 312-316.

[56] J. C. Pati and A. Salam. "Lepton Number as the Fourth "Color"." Phys. Rev. D 10 (1974) 275-289.

[57] H. Georgi and S. L. Glashow. "Unity of All Elementary-Particle Forces." Phys. Rev. Lett. 32 (1974) 438-441.

[58] S. Alekhin et al. "A Facility to Search for Hidden Particles at the CERN SPS: the SHiP Physics Case." (2015).

[59] C. Cheung et al. "Kinetic Mixing as the Origin of a Light Dark-Gauge-Group Scale." Phys. Rev. D 80 (2009) 035008.

[60] V. Braun, Y.-H. He, and B. Ovrut. "Supersymmetric Hidden Sectors for Heterotic Standard Models." Journal of High Energy Physics 2013 (2013) 8.

[61] M. Goodsell and A. Ringwald. "Light Hidden-Sector U(1)s in String Compactifications." Fortschritte der Physik 58 (2010) 716-720.

[62] S. Abel et al. "Kinetic Mixing of the Photon with Hidden U(1)s in String Phenomenology." Journal of High Energy Physics 2008 (2008) 124. 
[63] M. Pospelov. "Secluded U(1) below the Weak Scale." Phys. Rev. D 80 (2009) 095002.

[64] S. Gninenko and N. Krasnikov. "The Muon Anomalous Magnetic Moment and a New Light Gauge Boson." Physics Letters B 513 (2001) 119-122.

[65] G. W. Bennett et al. "Final Report of the E821 Muon Anomalous Magnetic Moment Measurement at BNL." Phys. Rev. D 73 (2006) 072003.

[66] J. Jaeckel, J. Redondo, and A. Ringwald. "Signatures of a Hidden Cosmic Microwave Background." Phys. Rev. Lett. 101 (2008) 131801.

[67] O. Adriani et al. "PAMELA Results on the Cosmic-Ray Antiproton Flux from $60 \mathrm{MeV}$ to $180 \mathrm{GeV}$ in Kinetic Energy." Phys. Rev. Lett. 105 (2010) 121101.

[68] M. Ackermann et al. "Measurement of Separate Cosmic-Ray Electron and Positron Spectra with the Fermi Large Area Telescope." Phys. Rev. Lett. 108 (2012) 011103.

[69] M. Aguilar et al. "First Result from the Alpha Magnetic Spectrometer on the International Space Station: Precision Measurement of the Positron Fraction in Primary Cosmic Rays of 0.5-350 GeV." Phys. Rev. Lett. 110 (2013) 141102.

[70] M. Pospelov and A. Ritz. "Astrophysical Signatures of Secluded Dark Matter." Physics Letters B 671 (2009) 391-397.

[71] J. Ruderman and T. Volansky. "Decaying into the Hidden Sector." Journal of High Energy Physics 2010 (2010) 24.

[72] M. Rocha et al. "Cosmological Simulations with Self-Interacting Dark Matter I. Constant-Density Cores and Substructure." Monthly Notices of the Royal Astronomical Society 430 (2013) 81-104. 
[73] A. H. G. Peter et al. "Cosmological Simulations with Self-Interacting Dark Matter II. Halo Shapes versus Observations." Monthly Notices of the Royal Astronomical Society 430 (2013) 105-120.

[74] S. Tulin, H.-B. Yu, and K. M. Zurek. "Beyond Collisionless Dark Matter: Particle Physics Dynamics for Dark Matter Halo Structure." Phys. Rev. D 87 (2013) 115007.

[75] M. G. Walker and J. Pearrubia. "A Method for Measuring (Slopes of) the Mass Profiles of Dwarf Spheroidal Galaxies." The Astrophysical Journal $\mathbf{7 4 2}$ (2011) 20 .

[76] J. F. Navarro, C. S. Frenk, and S. D. M. White. "A Universal Density Profile from Hierarchical Clustering." The Astrophysical Journal 490 (1997) 493.

[77] A. Falkowski et al. "Hidden Higgs Decaying to Lepton Jets." Journal of High Energy Physics 2010 (2010) 77.

[78] M. Baumgart et al. "Non-Abelian Dark Sectors and Their Collider Signatures." Journal of High Energy Physics 2009 (2009) 014.

[79] G. Aad et al. "Search for Long-Lived Neutral Particles Decaying into Lepton Jets in Proton-Proton Collisions at $\sqrt{s}=8 \mathrm{TeV}$ with the ATLAS Detector." Journal of High Energy Physics 2014 (2014) 88.

[80] J. D. Bjorken et al. "New Fixed-Target Experiments to Search for Dark Gauge Forces." Phys. Rev. D 80 (2009) 075018.

[81] J. D. Bjorken et al. "Search for Neutral Metastable Penetrating Particles Produced in the SLAC Beam Dump." Phys. Rev. D 38 (1988) 3375-3386.

[82] E. M. Riordan et al. "Search for Short-Lived Axions in an Electron-BeamDump Experiment." Phys. Rev. Lett. 59 (1987) 755-758. 
[83] A. Bross et al. "Search for Short-Lived Particles Produced in an Electron Beam Dump." Phys. Rev. Lett. 67 (1991) 2942-2945.

[84] S. D. Biller and S. M. Oser. "Another Look at Confidence Intervals: Proposal for a More Relevant and Transparent Approach." Nuclear Instruments and Methods in Physics Research Section A: Accelerators, Spectrometers, Detectors and Associated Equipment 774 (2015) 103-119.

[85] M. Endo, K. Hamaguchi, and G. Mishima. "Constraints on Hidden Photon Models from Electron $g-2$ and Hydrogen Spectroscopy." Phys. Rev. D 86 (2012) 095029.

[86] D. Babusci et al. "Limit on the Production of a Light Vector Gauge Boson in $\phi$ Meson Decays with the KLOE Detector." Physics Letters B 720 (2013) $111-115$.

[87] F. Archilli et al. "Search for a Vector Gauge Boson in $\phi$ Meson Decays with the KLOE Detector." Physics Letters B 706 (2012) 251-255.

[88] P. Adlarson et al. "Search for a Dark Photon in the $\pi^{0} \rightarrow e^{+} e^{-} \gamma$ Decay." Physics Letters B $\mathbf{7 2 6}$ (2013) 187-193.

[89] S. Abrahamyan et al. "Search for a New Gauge Boson in Electron-Nucleus Fixed-Target Scattering by the APEX Experiment." Phys. Rev. Lett. 107 (2011) 191804

[90] H. Merkel et al. "Search for Light Gauge Bosons of the Dark Sector at the Mainz Microtron.” Phys. Rev. Lett. 106 (2011) 251802.

[91] M. Reece and L.-T. Wang. "Searching for the Light Dark Gauge Boson in GeV-Scale Experiments." Journal of High Energy Physics 2009 (2009) 051. 
[92] B. Aubert et al. "Search for Dimuon Decays of a Light Scalar Boson in Radiative Transitions $\Upsilon \rightarrow \gamma A^{0}$." Phys. Rev. Lett. 103 (2009) 081803.

[93] J. B. Dent, F. Ferrer, and L. M. Krauss. "Constraints on Light Hidden Sector Gauge Bosons from Supernova Cooling." (2012).

[94] H. K. Dreiner et al. "Supernova Constraints on MeV Dark Sectors from $e^{+} e^{-}$ Annihilations." Phys. Rev. D 89 (2014) 105015.

[95] U. Ellwanger, C. Hugonie, and A. M. Teixeira. "The Next-to-Minimal Supersymmetric Standard Model." Physics Reports 496 (2010) 1-77.

[96] R. Dermíšek and J. F. Gunion. "Escaping the Large Fine-Tuning and Little Hierarchy Problems in the Next to Minimal Supersymmetric Model and $h \rightarrow a a$ Decays." Phys. Rev. Lett. 95 (2005) 041801.

[97] J. E. Kim and H. Nilles. "The $\mu$-Problem and the Strong CP-Problem." Physics Letters B 138 (1984) 150-154.

[98] A. Belyaev et al. "LHC Discovery Potential of the Lightest NMSSM Higgs boson in the $h_{1} \rightarrow a_{1} a_{1} \rightarrow 4 \mu$ Channel." Phys. Rev. D 81 (2010) 075021.

[99] R. Dermisek and J. F. Gunion. "New Constraints on a Light CP-Odd Higgs Boson and Related NMSSM Ideal Higgs Scenarios." Phys. Rev. D 81 (2010) 075003.

[100] V. Abazov et al. "Search for Next-to-Minimal Supersymmetric Higgs Bosons in the $h \rightarrow a a \rightarrow \mu \mu \mu \mu, \mu \mu \tau \tau$ Channels Using $p \bar{p}$ Collisions at $\sqrt{s}=1.96$ TeV." Phys. Rev. Lett. 103 (2009) 061801.

[101] G. Aad et al. "Search for Displaced Muonic Lepton Jets from Light Higgs Boson Decay in Proton-Proton Collisions at with the ATLAS Detector." Physics Letters B 721 (2013) 32-50. 
[102] S. Chatrchyan et al. "Search for a Non-Standard-Model Higgs Boson Decaying to a Pair of New Light Bosons in Four-Muon Final States." Physics Letters B 726 (2013) 564-586.

[103] V. Khachatryan et al. "Search for a Very Light NMSSM Higgs Boson Produced in Decays of the $125 \mathrm{GeV}$ Scalar Boson and Decaying into $\tau$ Leptons in pp Collisions at s = 8 TeV." Journal of High Energy Physics 2016 (2016) 1-46.

[104] G. Aad et al. "Search for New Light Gauge Bosons in Higgs Boson Decays to Four-Lepton Final States in $p p$ Collisions at $\sqrt{s}=8 \mathrm{TeV}$ with the ATLAS Detector at the LHC." Phys. Rev. D 92 (2015) 092001.

[105] W. Love et al. "Search for Very Light CP-Odd Higgs Boson in Radiative Decays of $\Upsilon(1 S) . " \quad$ Phys. Rev. Lett. 101 (2008) 151802.

[106] T. Aaltonen et al. "Search for a Very Light CP-Odd Higgs Boson in Top Quark Decays from $p \bar{p}$ Collisions at $\sqrt{s}=1.96$ TeV." Phys. Rev. Lett. 107 (2011) 031801.

[107] S. Chatrchyan et al. "Search for a Light Pseudoscalar Higgs Boson in the Dimuon Decay Channel in pp Collisions at $\sqrt{s}=7$ TeV." Phys. Rev. Lett. 109 (2012) 121801.

[108] R. Dermisek and J. F. Gunion. "New Constraints on a Light CP-Odd Higgs Boson and Related NMSSM Ideal Higgs Scenarios." Phys. Rev. D 81 (2010) 075003.

[109] A. Falkowski et al. "Hidden Higgs Decaying to Lepton Jets." Journal of High Energy Physics 2010 (2010) 77.

[110] J. Alwall et al. "The Automated Computation of Tree-Level and Next-toLeading Order Differential Cross Sections, and Their Matching to Parton 
Shower Simulations." Journal of High Energy Physics 2014 (2014) 1-157.

[111] P. Meade and M. Reece. "BRIDGE: Branching Ratio Inquiry / Decay Generated Events." (2007).

[112] T. Sjostrand, S. Mrenna, and P. Z. Skands. "PYTHIA 6.4 Physics and Manual." JHEP 05 (2006) 026.

[113] C. Balazs, H.-J. He, and C. Yuan. "QCD Corrections to Scalar Production via Heavy Quark Fusion at Hadron Colliders." Phys. Rev. D60 (1999) 114001.

[114] U. Ellwanger, J. F. Gunion, and C. Hugonie. "NMHDECAY: A Fortran Code for the Higgs Masses, Couplings and Decay Widths in the NMSSM." Journal of High Energy Physics 2005 (2005) 066.

[115] M. Spira. "HIGLU: A Program for the Calculation of the Total Higgs Production Cross-Section at Hadron Colliders via Gluon Fusion Including QCD Corrections." (1995).

[116] S. Agostinelli et al. "Geant4-A Simulation Toolkit." Nuclear Instruments and Methods in Physics Research Section A: Accelerators, Spectrometers, Detectors and Associated Equipment 506 (2003) 250-303.

[117] A. Augusto et al. "The LHCb Detector at the LHC." Journal of Instrumentation 3 (2008) S08005.

[118] K. Aamodt et al. "The ALICE Experiment at the CERN LHC." Journal of Instrumentation 3 (2008) S08002.

[119] B. Wolf. Handbook of Ion Sources. Taylor \& Francis, 1995.

[120] M. Lamont. "Status of the LHC." Journal of Physics: Conference Series 455 (2013) 012001. 
[121] CMS Collaboration. "Description and Performance of Track and PrimaryVertex Reconstruction with the CMS Tracker." JINST (2014).

[122] "The CMS High Level Trigger." The European Physical Journal C - Particles and Fields 46 (2006) 605-667.

[123] CMS Collaboration. "Particle-Flow Event Reconstruction in CMS and Performance for Jets, Taus, and MET." (2009).

[124] CMS Collaboration. "Commissioning of the Particle-Flow Reconstruction in Minimum-Bias and Jet Events from pp Collisions at 7 TeV." (2010).

[125] P. Billoir. "Progressive Track Recognition with a Kalman Like Fitting Procedure." Comput. Phys. Commun. (1989).

[126] CMS Collaboration. "Performance of CMS Muon Reconstruction in pp Collision Events at $\sqrt{(s)}=7$ TeV." Journal of Instrumentation 7 (2012) P10002.

[127] K. Rose. "Deterministic Annealing for Clustering, Compression, Classification, Regression and related Optimisation Problems." Proceedings of the IEEE (1998).

[128] P. V. R. Fruehwirth, W. Waltenberger. "Adaptive Vertex Filtering." $C M S$ Note (2007).

[129] "Electron Reconstruction and Identification at $\sqrt{s}=7 \mathrm{TeV}$." Technical Report CMS-PAS-EGM-10-004, CERN, Geneva, 2010.

[130] G. L. Bayatian et al. CMS Physics: Technical Design Report Volume 1: Detector Performance and Software. Technical Design Report CMS. CERN, Geneva, 2006.

[131] E. Meschi et al. "Electron Reconstruction in the CMS Electromagnetic Calorimeter." Technical Report CMS-NOTE-2001-034, CERN, Geneva, 2001. 
[132] W. Adam et al. "Reconstruction of Electrons with the Gaussian-Sum Filter in the CMS Tracker at the LHC." Technical Report CMS-NOTE-2005-001, CERN, Geneva, 2005.

[133] R. Fruhwirth. "Application of Kalman Filtering to Track and Vertex Fitting." Nucl. Instrum. Meth. A262 (1987) 444-450.

[134] M. Cacciari, G. P. Salam, and G. Soyez. "The Anti- $k_{t}$ Jet Clustering Algorithm." Journal of High Energy Physics 2008 (2008) 063.

[135] M. Schrder. "Performance of Jets at CMS." J. Phys. Conf. Ser. 587 (2015) 012004.

[136] "Pileup Jet Identification." Technical Report CMS-PAS-JME-13-005, CERN, Geneva, 2013.

[137] CMS collaboration. "Determination of Jet Energy Calibration and Transverse Momentum Resolution in CMS." Journal of Instrumentation 6 (2011) P11002.

[138] CMS Collaboration. "Performance of $\tau$-Lepton Reconstruction and Identification in CMS." Journal of Instrumentation 7 (2012) P01001.

[139] CMS Collaboration. "MET Performance in 8 TeV Data." Technical Report CMS-PAS-JME-12-002, CERN, Geneva, 2013.

[140] J. Pivarski, A. Safonov, and A. Tatarinov. "Search for Collimated Groups of Muons." CMS Note 2010/462 (2010).

[141] G. C. Hill et al. "Examining the Balance Between Optimising an Analysis for Best Limit Setting and Best Discovery Potential." In "Statistical problems in particle physics, astrophysics and cosmology. Proceedings, Conference, PHYSTAT05, Oxford, UK, September 12-15, 2005," 2005 108-111. 
[142] G. C. Hill and K. Rawlins. "Unbiased Cut Selection for Optimal Upper Limits in Neutrino Detectors: The Model Rejection Potential Technique." Astroparticle Physics 19 (2003) 393-402.

[143] A. York et al. "Measurement of Double $J / \psi$ Production Cross-Section in pp Collisions at $\sqrt{s}=7$ TeV." CMS Note 2012/222 (2012).

[144] CMS Collaboration. "Absolute Calibration of Luminosity Measurement at CMS: Summer 2011 Update." Technical Report CMS-PAS-EWK-11-001, CERN, Geneva, 2011.

[145] D. Acosta et al. "Updated Search for New Physics Using Highly Boosted Z Decaying to Dimuons." CMS Analysis Note 2011/141 (2011).

[146] A. Gritsan et al. "Search for a Higgs Boson in the Decay Channel $H \rightarrow Z Z^{*} \rightarrow$ 4l." CMS Analysis Note 11/387 (2011).

[147] S. Chatrchyan et al. "Search for the Standard Model Higgs Boson in the Decay Channel $\mathrm{H}$ to ZZ to 4 Leptons in pp Collisions at sqrt(s) $=7$ TeV." Phys. Rev. Lett. 108 (2012) 111804.

[148] S. Dittmaier et al. "Handbook of LHC Higgs Cross Sections: 1. Inclusive Observables.", 2011.

[149] R. D. Cousins and V. L. Highland. "Incorporating Systematic Uncertainties into an Upper Limit." Nuclear Instruments and Methods in Physics Research Section A: Accelerators, Spectrometers, Detectors and Associated Equipment 320 (1992) 331-335.

[150] A. Falkowski et al. "Hidden Higgs Decaying to Lepton Jets." JHEP 05 (2010) 077. 
[151] V. M. Abazov et al. "Search for NMSSM Higgs Bosons in the $h \rightarrow a a \rightarrow$ $\mu \mu \mu \mu, \mu \mu \tau \tau$ Channels Using p Anti-p Collisions at $\sqrt{s}=1.96 \mathrm{TeV} . " \quad$ Phys. Rev. Lett. 103 (2009) 061801.

[152] S. Chatrchyan et al. "Search for Light Resonances Decaying into Pairs of Muons as a Signal of New Physics." JHEP 07 (2011) 098. 